\title{
SYMMETRIES OF PLANAR GROWTH FUNCTIONS. II
}

\author{
WILLIAM J. FLOYD
}

\begin{abstract}
Let $G$ be a finitely generated group, and let $\Sigma$ be a finite generating set of $G$. The growth function of $(G, \Sigma)$ is the generating function $f(z)=$ $\sum_{n=0}^{\infty} a_{n} z^{n}$, where $a_{n}$ is the number of elements of $G$ with word length $n$ in $\Sigma$. Suppose that $G$ is a cocompact group of isometries of Euclidean space $\mathbb{E}^{2}$ or hyperbolic space $\mathbb{H}^{2}$, and that $D$ is a fundamental polygon for the action of $G$. The full geometric generating set for $(G, D)$ is $\{g \in G: g \neq$ 1 and $g D \cap D \neq \varnothing\}$. In this paper the recursive structure for the growth function of $(G, \Sigma)$ is computed, and it is proved that the growth function $f$ is reciprocal $(f(z)=f(1 / z))$ except for some exceptional cases when $D$ has three, four, or five sides.
\end{abstract}

If $G$ is a finitely generated group and $\Sigma$ is a finite generating set for $G$, then the word norm $|\cdot|_{G, \Sigma}=|\cdot|$ is the norm on $G$ defined by

$$
|g|=\min \left\{n: g=g_{1} \cdots g_{n} \text { and } g_{i} \in \Sigma \text { or } g_{i}^{-1} \in \Sigma \text { for each } i \in\{1, \ldots, n\}\right\} \text {. }
$$

The growth series of $G$, with respect to $\Sigma$, is

$$
h(z)=h_{G, \Sigma}(z)=\sum_{g \in G} z^{|g|}=\sum_{n=0}^{\infty} s_{n} z^{n},
$$

where $s_{n}$ is the number of elements of $G$ of word norm $n$. If $G$ is a cocompact, discrete group of isometries of hyperbolic $n$-space $\mathbb{H}^{n}$, then Cannon [2] showed that, for any finite generating set $\Sigma$ for $G$, the growth series $h_{G, \Sigma}$ is the Maclaurin series of a rational function $f_{G, \Sigma}$. We call $f_{G, \Sigma}$ the growth function of $G$ with respect to $\Sigma$, and often denote it by $f$. Cannon showed that the growth series was rational by showing that there is a linear recursion on the group. That is, he showed that there is a positive integer $r$, a function $\tau: G \backslash\{e\} \rightarrow\{1, \ldots, r\}$, and an $r \times r$ matrix $A$, called the recursion matrix, such that if $V$ (the initial vector) is the $r \times 1$ matrix with $i$ th entry the number of elements $g \in G$ with $|g|=1$ and $\tau(g)=i$, then for every positive integer $n$, the $i$ th entry of $A^{n-1} V$ is the number of elements $g \in G$ with $|g|=n$ and $\tau(g)=i$. (We will usually write that $g$ has type $\tau_{i}$ if $\tau(g)=i$.) In [1], Benson showed that, if $G$ is a cocompact, discrete group of isometries of Euclidean $n$-space $\mathbb{E}^{n}$ and $\Sigma$ is a finite generating set for $G$, then the growth series of $(G, \Sigma)$ is the Maclaurin series of a rational function.

Received by the editors June 25, 1991 .

1991 Mathematics Subject Classification. Primary 20F05; Secondary 22E40, 11F06, 57N05.

Supported in part by NSF Grants DMS-8701419 and DMS-8902199. 
If $G$ is a cocompact, discrete group of isometries of $X$, where $X=\mathbb{E}^{2}$ or $X=\mathbb{H}^{2}$, and $x \in X$ is a basepoint that is not fixed by any nontrivial element of $G$, then the Dirichlet domain $D=D_{x}=\{y \in X: d(x, y) \leq$ $d(g x, y)$ for all $g \in G\}$. Then $D$ is a compact polygon and is a fundamental domain for the action of $G$. The set of edge-pairing elements $\Sigma=\{g \in G$ : $g D \cap D$ is 1 -dimensional $\}$ is the geometric generating set for $(G, D)$. Cannon [3] and Wagreich [7] computed an infinite family of growth functions of surface groups with geometric generating sets. In all of their examples, the denominator of the growth function $f$ is a Salem polynomial times a product of cyclotomic polynomials, $f(1)=1 / \chi(G)$, and $f$ is reciprocal $(f(z)=f(1 / z)$ if $z \neq 0)$.

In [4 and 5], Floyd and Plotnick showed that if $f$ is a growth function, with respect to a geometric generating set, for a cocompact, discrete group of isometries of $\mathbb{H}^{2}$, then $f(1)=1 / \chi(G)$ unless 1 is an eigenvalue of the recursion matrix $A$. In [5], they showed that, if $f$ is a growth function, with respect to a geometric generating set, of a cocompact, discrete group of isometries of $\mathbb{E}^{2}$ or $\mathbb{H}^{2}$, then $f$ is reciprocal exactly if $G$ has no maximal element $g$ with respect to the word norm. An element $g$ is maximal if there is no generator $g_{i} \in \Sigma$ with $\left|g g_{i}\right|=|g|+1$ or $\left|g g_{i}^{-1}\right|=|g|+1$. The element $g$ is maximal if and only if $g D$ does not contain an edge in the boundary of the combinatorial ball $B(|g|)=\bigcup\{a D: a \in G$ and $|a| \leq|g|\}$. (For this reason, they used the terminology "buried domain" for this phenomenon.) There were also examples in [5] that showed that it is not generally true that the denominator of $f$ is a product of cyclotomic polynomials and a Salem polynomial.

- There is another natural generating set associated to a Dirichlet domain for a cocompact, discrete group of isometries. If $G$ is a discrete group of isometries of $\mathbb{E}^{n}$ or $\mathbb{H}^{n}$ and $D$ is a Dirichlet domain for the action of $G$, then the full geometric generating set for $(G, D)$ is $\{g \in G: g \neq 1$ and $g D \cap D \neq$ $\varnothing\}$. I first started thinking about these generating sets while studying growth functions of cocompact, discrete groups of isometries of $\mathbb{H}^{3}$. It is harder in higher dimensions to show that combinatorial balls are homeomorphic to balls, and Matt Grayson and Bill Thurston suggested these generating sets since the combinatorial balls are often convex.

In this paper we study the growth functions, with full geometric generating sets, of cocompact, discrete groups of isometries of $\mathbb{E}^{2}$ or $\mathbb{H}^{2}$. If $G$ is a cocompact, discrete group of isometries of $\mathbb{E}^{2}$ or $\mathbb{H}^{2}$ and $D$ is a Dirichlet domain for $D$, then a domain $g D, g \in G$, is a buried domain if $g D \subset$ $\operatorname{int}(B(|g|))$. Equivalently, $g D$ is a buried domain if $g$ is a maximal element of $G$ with respect to the word norm of the full geometric generating set for $(G, D)$. Our main result is the following:

Theorem. Let $G$ be a cocompact, discrete group of isometries of $\mathbb{E}^{2}$ or $\mathbb{H}^{2}$, let $D$ be a Dirichlet region for the action of $G$, and let $f$ be the growth function of $G$ with respect to the full geometric generating set. Then either there are no buried domains and $f$ is reciprocal or there are buried domains and $f$ is not reciprocal. The denominator of $f$ is reciprocal even if there are buried domains. The cases for which there are buried domains are the following:

(i) $D$ is a triangle with a vertex of valence 3 or 4;

(ii) $D$ is a quadrilateral with vertices of valence $3,2 r, 3$, and $2 r$ (in cyclic order), for some $r \geq 3$; 
(iii) $D$ is a quadrilateral with vertices of valence $3,2 r, 4$, and $2 r$ (in cyclic order), for some $r \geq 4$; or

(iv) $D$ is a pentagon with vertices of valence $3,3, q, 3$, and $r$ (in cyclic order), with $1 / q+1 / r \leq 1 / 2$.

We denote by $\Delta^{*}(p, q, r)$ the Coxeter triangle group generated by reflections in the sides of a triangle with angles $\pi / p, \pi / q$, and $\pi / r$, and by $\Delta(p, q, r)$ its orientation-preserving subgroup whose elements are products of an even number of reflections. In case (i), $G$ is a group with the same tessellation as $\Delta^{*}(2, q, r)$ or $\Delta(3, q, r)$, in case (ii) $G=\Delta(3,3, r)$, in case (iii) $G=\Delta(3,4, r)$, and in case (iv) $G=\Delta(2, q, r)$. Figure 1 shows how these fundamental domains arise for these groups.

The first three sections treat the case where either $D$ has at least six sides, $D$ has five sides and does not have three adjacent vertices with valence 3 , or $D$ has four sides and no vertices of valence 3 . In $\S 1$ we show that for these cases, the combinatorial balls $B(n)$ are homeomorphic to balls and a vertex of $\partial B(n)$ has valence 1 or 2 in $B(n)$. In $\S 2$ we construct the recursion matrix $A$, initial vector $V$, and weight vector $U$ for these cases, and construct the inverse of the recursion matrix. In $\S 3$ we prove that the growth function is reciprocal for these cases. We do this by proving that $U A^{n} W=-U A^{-n} W$ for all nonnegative integers $n$, where $W=A^{-1} V$.

If $D$ is a triangle, then there will be vertices of $\partial B(n)$ with valence 3 in $B(n)$, and the above analysis does not apply. Section 4 computes the growth functions for all cases with $D$ a triangle, and shows that the growth function is reciprocal exactly if there are no buried domains, and that there are buried domains if and only if $D$ has a vertex of valence 3 or 4 . In particular, there are Coxeter groups with buried domains and growth functions that are not reciprocal.

If $D$ is a quadrilateral with a vertex of valence 3 , then either there are buried domains or there are vertices of $\partial B(n)$ with valence 3 in $B(n)$. The growth functions for these cases are computed in $\S 5$. There are buried domains exactly if $G$ is a triangle group $\Delta(3,3, r)$ or $\Delta(3,4, r)$, and these are the cases where the growth function is not reciprocal.

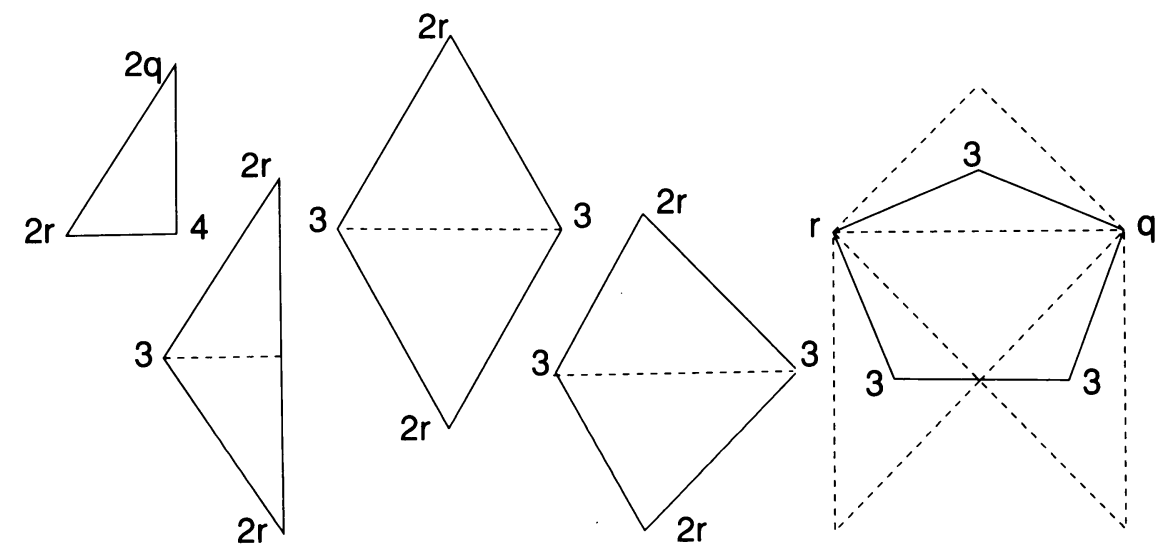

FIGURE 1. The cases with buried domains 
If $D$ is a pentagon with 3 vertices of valence 3 that are not adjacent, then $G$ is a triangle group $\Delta(2, q, r)$ and there are buried domains. The growth functions for these are computed in $\S 6$ and they are not reciprocal.

Since the combinatorial balls are homeomorphic to balls and there is a linear recursion on the boundary vertices of the combinatorial balls, if $G$ acts on $\mathbb{H}^{2}$ it is easy to adapt the argument from [4] to show that $f(1)=1 / \chi(G)$ if 1 is not an eigenvalue of the recursion matrix $A$. This is done in $\S 7$. It is not always true that $f(1)=1 / \chi(G)$.

Finally, in $\S 8$ we list the growth functions, with full geometric generating sets, for the fundamental group of a closed nonorientable surface of genus three and of a closed orientable surface of genus 2 .

The above theorem is very similar to the planar reciprocity theorem in [5] in that the growth function is reciprocal exactly if there are no buried domains. However, a key ingredient of the proof of the planar reciprocity theorem is the reciprocity of the growth functions, with geometric generating sets, of finite Coxeter groups. The growth functions of finite Coxeter groups are not always reciprocal with respect to the full geometric generating sets, and some of the cocompact planar groups whose growth functions, with respect to full geometric generating sets, are not reciprocal are Coxeter groups.

It is easy to construct examples of finite generating sets for cocompact planar groups for which the growth function is not reciprocal and there are no maximal elements with respect to the word norm. If $x$ and $y$ are generators for $\mathbb{Z}^{2}$ and $n$ and $m$ are positive integers, then $\Sigma=\left\{x, x^{2}, \ldots, x^{n}, y, y^{2}, \ldots, y^{m}\right\}$ is a generating set for $\mathbb{Z}^{2}$ without maximal elements. The growth function for $\left(\mathbb{Z}^{2}, \Sigma\right)$

$$
f(z)^{\prime}=\frac{1+(2 n-1) z}{1-z} \cdot \frac{1+(2 m-1) z}{1-z},
$$

is not reciprocal if $n \neq 1$ or $m \neq 1$.

The computations of growth functions in $\S \S 4,5,6$, and 8 were done using Fadeev's algorithm (see [6]) to compute $(I-z A)^{-1}$. While this is not the most efficient algorithm to compute the growth function, it is well adapted to the symbolic arithmetic needed in $\S \S 4,5$, and 6 , which was done using MACSYMA.

\section{COMBINATORICS OF THE COMBINATORIAL BALLS}

Let $G$ be a cocompact, discrete group of isometries of Euclidean 2-space $\mathbb{E}^{2}$ or hyperbolic 2-space $\mathbb{H}^{2}$, and let $D$ be a Dirichlet fundamental domain for the action of $G$. The full geometric generating set for $(G, D)$ is $\Sigma=\{g \in G$ : $g D \cap D \neq \varnothing$ and $g \neq 1\}$. If $v$ is a vertex of the tessellation $\{g D: g \in G\}$, then the valence of $v$ is $\#\{g \in G: v \in g D\}$. For each integer $n \geq 0$, the combinatorial ball $B(n)=\bigcup\left\{g D: g \in G\right.$ and $\left.|g|_{\Sigma} \leq n\right\}$. If $v$ is a vertex in $\partial B(n)$, the valence of $v$ in $B(n)$ is $\#\left\{g \in G:|g|_{\Sigma} \leq n\right.$ and $\left.v \in g D\right\}$. If $v$ and $w$ are vertices in the tessellation $\{g D: g \in G\}$ and there is an element $g \in G$ with $g(v)=w$, then we say that $v$ is $G$-equivalent to $w$ and we write $v \sim_{G} w$.

As in [4 and 5], it is important to know that each combinatorial ball is homeomorphic to a ball. As in [5], symmetry of the growth function depends upon the absence of buried domains. Since one is using the full geometric generating set, buried domains look different geometrically. A domain $g D$ is buried if 
there is no $h \in G$ such that $\left|g^{-1} h\right|=1$ and $|h|=|g|+1$. The proof that combinatorial balls are homeomorphic to balls is much easier with the full geometric generating set rather then the geometric generating set. If $D$ has at least four edges and each angle of $D$ has measure at most $\pi / 2$, then the combinatorial balls are convex. However, buried domains occur more frequently with this larger generating set.

In this section we show that if $D$ has at least six sides, $D$ has four sides and no vertices of valence 3 , or $D$ has five sides and $D$ does not have three vertices which have valence 3 and are not adjacent, then for all nonnegative integers $n$, $B(n)$ is homeomorphic to a ball and each vertex of $\partial B(n)$ has valence 1 or 2 in $B(n)$. We will do this by using the following lemma.

Lemma 1.1. Let $G$ be a cocompact, discrete group of isometries of $\mathbb{E}^{2}$ or $\mathbb{H}^{2}$, let $D$ be a Dirichlet region for the action of $G$, and let $\Sigma$ be the full geometric generating set for $(G, D)$. Let $n$ be a nonnegative integer which satisfies the following conditions:

(i) $B(n)$ is homeomorphic to a ball;

(ii) if $g \in G$ with $|g|=n+1$, then $g D \cap B(n)$ is connected; and

(iii) if $g, h \in G$ with $|g|=|h|=n+1$ and $g D \cap h D \neq \varnothing$, then $g D \cap h D$ contains a vertex of $\partial B(n)$.

Then $B(n+1)$ is homeomorphic to a ball and each vertex of $\partial B(n+1)$ has valence 1 or 2 in $B(n+1)$.

Proof. Let $e_{1}, \ldots, e_{p}$ be an enumeration of the edges of $\partial B(n)$ and let $v_{1}, \ldots$, $v_{p}$ be an enumeration of the vertices of $\partial B(n)$. Define $C_{0}, \ldots, C_{p}$ recursively by $C_{0}=B(n)$ and, for $i>0, C(i)=C(i-1) \cup g_{i} D$ where $g_{i} \in G$ with $\left|g_{i}\right|=$ $n+1$ and $e_{i} \subset g_{i} D$. Now define $C_{p+1}, \ldots, C_{2 p}$ recursively by $C_{i}=C_{i-1} \cup$ $\left\{g D: g \in G,|g|=n+1\right.$, and $\left.g D \cap B(n)=v_{i-p}\right\}$. Then $C(i)=C(i-1)$ or $C(i)$ is obtained from $C(i-1)$ by attaching a ball along a subset homeomorphic to an interval, so it follows inductively that each $C_{i}$ is homeomorphic to a ball. Hence $B(n+1)=C_{2 p}$ is homeomorphic to a ball. It follows from (iii) that each vertex of $\partial B(n+1)$ has valence 1 or 2 in $B(n+1)$.

Lemma 1.2. Let $X$ be $\mathbb{E}^{2}$ or $\mathbb{H}^{2}$, and let $G$ be a cocompact, discrete group of isometries of $X$. Let $D$ be a Dirichlet region for the action of $G$, and suppose that either $D$ has at least six edges or that $D$ has at least four edges and no vertex of $D$ has valence 3 . Then the following conditions hold for every nonnegative integer $n$ :

(i) $B(n)$ is homeomorphic to a ball;

(ii) if $g \in G$ with $|g|=n+1$, then $g D \cap B(n)$ is connected;

(iii) if $g, h \in G$ with $|g|=|h|=n+1$ and $g D \cap h D \neq \varnothing$, then $g D \cap h D$ contains a vertex of $\partial B(n)$;

(iv) each vertex of $\partial B(n)$ has valence 1 or 2 in $B(n)$; and

(v) if $e$ is an edge in $\partial B(n)$ and $D$ has at least five edges, then one of the vertices of $\partial e$ has valence 1 in $B(n)$.

Proof. Since the conditions of the lemma are combinatorial, it suffices to assume that if $v$ is a vertex of $D$, then the measure of the angle at $v$ is $2 \pi$ divided by the valence of $v$. Let $m$ be the number of edges in $D$, and let $\theta$ be the 
minimum measure of an angle of $D$. Let $K=0$ if $X=\mathbb{E}^{2}$ and let $K=1$ if $X=\mathbb{H}^{2}$.

First suppose that $m \geq 6$. We will use Lemma 1.1 to prove the lemma by induction. All five conditions are obvious for $n=0$. Assume that $n \geq 0$ and (i)-(v) hold for $n$. Let $g \in G$ with $|g|=n+1$. We prove by contradiction that $g D \cap B(n)$ is connected. Suppose not. Then there is a polygon $C$ such that $\operatorname{int}(C)$ is a component of $X-(g D \cup B(n))$. Let $r$ be the number of edges of $\partial C$ which are in $g D$ and let $t$ be the number of edges of $\partial C$ which are in $B(n)$. $C$ has $r+t$ vertices. The two vertices of $C$ in $g D \cap B(n)$ each have angle measure at least $\theta$. The $r-1$ vertices of $C$ in $g D-B(n)$ each have angle measure at least $4 \pi / 3$. By $(\mathrm{v})$, the sum of the angle measures of the $t-1$ vertices of $C$ in $B(n)-g D$ is at least $(t-1) \pi-\pi / 3$. By the Gauss-Bonnet formula,

$$
\begin{aligned}
K \cdot \operatorname{area}(C) & =\pi(r+t-2)-\text { angle sum of vertices of } B(n) \\
& \leq \pi(r+t-2)-\frac{(r-1) 4 \pi}{3}-(t-1) \pi+\frac{\pi}{3}-2 \theta \\
& =\pi\left(-\frac{r}{3}+\frac{2}{3}-\frac{2 \theta}{\pi}\right) .
\end{aligned}
$$

If $X=\mathbb{E}^{2}$, then $m=6$ and each angle of $D$ has measure $2 \pi / 3$, so $X=\mathbb{H}^{2}$ and $r=1$. Hence

$$
\begin{aligned}
\pi\left(-\frac{1}{3}+\frac{2}{3}-\frac{2 \theta}{\pi}\right) & \geq \operatorname{area}(C) \geq \operatorname{area}(D) \geq \pi(m-2)-(m-1) \frac{2 \pi}{3}-\theta \\
& =\pi\left(\frac{m}{3}-\frac{4}{3}-\frac{\theta}{\pi}\right),
\end{aligned}
$$

so $1 / 3 \geq(m-4) / 3+\theta / \pi$. Since $m \geq 6$, this is a contradiction and so $g D \cap B(n)$ is connected, proving (ii) for $n+1$.

Now suppose that $g, h \in G$ with $|g|=|h|=n+1, g \neq h$, and $g D \cap h D \neq$ $\varnothing$. We will prove by contradiction that $g D \cap h D$ contains a vertex of $\partial B(n)$. Suppose not. Then there is a polygon $C$ such that $\operatorname{int}(C)$ is a component of $X-(g D \cup h D \cup B(n))$. Let $r$ be the number of edges of $C$ in $g D$, let $s$ be the number of edges of $C$ in $h D$, and let $t$ be the number of edges of $C$ in $B(n)$. Then $r, s, t>0$ and we can assume without loss of generality that $r \geq s$. By the Gauss-Bonnet formula,

$$
\begin{aligned}
K \cdot \operatorname{area}(C) & \leq\left(\pi(r+s+t-2)-\frac{4 \pi}{3}(r+s-2)-(t-1) \pi+\frac{\pi}{3}-3 \theta\right) \\
& =\pi\left(-\frac{r}{3}-\frac{s}{3}+2-\frac{3 \theta}{\pi}\right),
\end{aligned}
$$

so $\theta<2 \pi / 3$ and $X=\mathbb{H}^{2}$. Hence

$$
\pi\left(-\frac{r}{3}-\frac{s}{3}+2-\frac{3 \theta}{\pi}\right) \geq \operatorname{area}(C) \geq r \cdot \operatorname{area}(D) \geq r \pi\left(\frac{m-4}{3}-\frac{\theta}{\pi}\right),
$$

and

$$
2 \geq r\left(\frac{m-3}{3}-\frac{\theta}{\pi}\right)+\frac{s}{3}+\frac{3 \theta}{\pi} .
$$

This is impossible unless $r=s=1$ and area $(C)=\operatorname{area}(D)$. But then $C$ is not convex, since $t=m-r-s \geq 4$. Thus $g D \cap h D$ contains a vertex of $\partial B(n)$, 
proving (iii) for $n+1$. By Lemma 1.1, (i) and (iv) hold for $n+1$. If $g \in G$ and $|g|=n+1$, then $g D$ has at most two edges in common with $B(n)$ and hence $g D$ has at least two edges in $\partial B(n+1)$. This establishes $(v)$ for $n+1$. The proof for $m \geq 6$ now follows by induction.

Now assume that $m=4$ or $m=5$ and that each angle of $D$ has measure at most $\pi / 2$. We will again prove that conditions (i) $-(\mathrm{v})$ hold for all nonnegative integers $n$. The conditions are clear if $n=0$, so assume that (i)-(v) hold for a nonnegative integer $n$. Then each angle of the polygon $B(n)$ has measure at most $\pi$ and $B(n)$ is convex. If $g \in G$ with $|g|=n+1$, then $g D \cap B(n)$ is convex and hence is connected, so (ii) is true for $n+1$. Now suppose that $g, h \in G$ with $|g|=|h|=n+1, g \neq h$, and $g D \cap h D \neq \varnothing$. We will prove by contradiction that $g D \cap h D$ contains a vertex of $\partial B(n)$. Suppose not. Then there is a polygon $C$ such that $\operatorname{int}(C)$ is a component of $X-(g D \cup h D \cup B(n))$. Let $r$ be the number of edges of $C$ in $g D$, let $s$ be the number of edges of $C$ in $h D$, and let $t$ be the number of edges of $C$ in $B(n)$. Then $r, s, t>0$ and we can assume without loss of generality that $r \geq s$. By the Gauss-Bonnet formula,

$$
\begin{aligned}
K \cdot \operatorname{area}(C) & \leq\left(\pi(r+s+t-2)-\frac{3 \pi}{2}(r+s-2)-(t-1) \pi-3 \theta\right) \\
& =\pi\left(2-\frac{r}{2}-\frac{s}{2}-\frac{3 \theta}{\pi}\right) .
\end{aligned}
$$

If $G$ acts on $\mathbb{E}^{2}$ then each angle of $D$ has measure $\pi / 2$ and we have a contradiction. So $G$ acts on $\mathbb{H}^{2}$ and $r+s \leq 3$. If $r=2$ and $s=1$, then

$$
\pi\left(2-1-\frac{1}{2}-\frac{3 \theta}{\pi}\right) \geq \operatorname{area}(C) \geq 3 \cdot \operatorname{area}(D) \geq 3 \pi\left(\frac{m-3}{2}-\frac{\theta}{\pi}\right),
$$

which gives a contradiction. It follows that $r=1$ and $s=1$, and

$$
\pi\left(1-\frac{3 \theta}{\pi}\right) \geq \operatorname{area}(C) \geq \operatorname{area}(D) \geq \pi\left(\frac{m-3}{2}-\frac{\theta}{\pi}\right) .
$$

Thus $m=4$ and area $(D)=\operatorname{area}(C)$. But this is impossible, since it implies that $t=2$ and hence one of the angles of $D$ has measure at least $\pi$. Thus $g D \cap h D$ contains a vertex of $\partial B(n)$, and (iii) holds for $n+1$. It now follows from Lemma 1.1 that (i) and (iv) hold for $n+1$. Because $D$ has no angle of measure $2 \pi / 3$, if $g \in G$ and $|g|=n+1$ then $g D$ has at most one edge in common with $B(n)$. If $D$ has five edges, $g D$ has at least two edges in $\partial B(n+1)$, proving ( $\mathrm{v}$ ) for $n+1$. The proof follows by induction.

Lemma 1.3. Let $G$ be a cocompact, discrete group of isometries of $\mathbb{H}^{2}$. Let $D$ be a Dirichlet region for the action of $G$, and suppose that $D$ has five edges and that exactly one of the vertices of $D$ has valence 3 . Then the following conditions hold for every nonnegative integer $n$ :

(i) $B(n)$ is homeomorphic to a ball;

(ii) if $g \in G$ with $|g|=n+1$, then $g D \cap B(n)$ is connected;

(iii) if $g, h \in G$ with $|g|=|h|=n+1$ and $g D \cap h D \neq \varnothing$, then $g D \cap h D$ contains a vertex of $\partial B(n)$;

(iv) each vertex of $\partial B(n)$ has valence 1 or 2 in $B(n)$; and 
(v) if $e$ is an edge in $\partial B(n)$ and one of the vertices of $\partial e$ has angle measure $4 \pi / 3$ in $B(n)$, then the other vertex of $\partial e$ has valence 1 in $B(n)$.

Proof. As before, we will assume that the measure of an angle of $D$ is $2 \pi$ divided by the valence of the vertex at which it is based. All five conditions are clear for $n=0$, so assume that $n \geq 0$ and (i)-(v) hold for $n$. By the Gauss-Bonnet formula, area $(D) \geq 3 \pi-2 \pi / 3-3 \pi / 2-\theta=5 \pi / 6-\theta$. Suppose that $g \in G$ with $|g|=n+1$ and $g D \cap B(n)$ not connected, and let $C, r$, and $t$ be chosen as in the proof of Lemma 1.2. The sum of the angle measures of the $(t-1)$ vertices of $C-g D$ is at least $(t-1) \pi-\pi / 3$, so

$$
\operatorname{area}(C) \leq \pi(r+t-2)-\frac{4 \pi}{3}(r-1)-(t-1) \pi+\frac{\pi}{3}-2 \theta=\pi\left(-\frac{r}{3}+\frac{2}{3}-\frac{2 \theta}{\pi}\right) .
$$

Since $\operatorname{area}(C) \geq \operatorname{area}(D),-r / 3+2 / 3-2 \theta / \pi \geq 5 / 6-\theta / \pi$. Since this is impossible, if $g \in G$ with $|g|=n+1$ then $g(D) \cap B(n)$ is connected, and (ii) is true for $n+1$.

Now suppose that $g, h \in G$ with $|g|=|h|=n+1, g \neq h$, and $g D \cap h D \neq$ $\varnothing$, and that $g D \cap h D$ does not contain a vertex of $\partial B(n)$. Let $C, r, s$, and $t$ be chosen as in the proof of Lemma 1.2. Then

$$
\begin{aligned}
\operatorname{area}(C) & \leq \pi(r+s+t-2)-\frac{4 \pi}{3}(r+s-2)-(t-1) \pi+\frac{\pi}{3}-3 \theta \\
& =\pi\left(-\frac{r}{3}-\frac{s}{3}+2-\frac{3 \theta}{\pi}\right) .
\end{aligned}
$$

Thus

$$
2 \cdot \operatorname{area}(D) \geq \pi\left(\frac{5}{3}-\frac{2 \theta}{\pi}\right)>\pi\left(2-\frac{r}{3}-\frac{s}{3}-\frac{3 \theta}{\pi}\right) \geq \operatorname{area}(C),
$$

and hence $C=D$. This implies $r=1, s=1$, and $t=3$, contradicting the fact that each angle of $D$ has measure at most $2 \pi / 3$. Thus $g D \cap h D$ contains a vertex of $\partial B(n)$ and (iii) is true for $n+1$. By Lemma 1.1, conditions (i) and (iv) hold for $n+1$. Now suppose that $e$ is an edge of $\partial B(n+1)$ such that both vertices of $\partial e$ have valence 2 in $B(n+1)$, and let $g \in G$ with $|g|=n+1$ and $e \subset g D$. Then $g D \cap B(n)$ must contain two edges of $g D$. This implies that the vertex of $g D$ with angle measure $2 \pi / 3$ is a vertex of $\partial B(n+1)$, and hence neither element of $\partial e$ has angle measure $4 \pi / 3$ in $\partial B(n+1)$. This establishes (v) for $n+1$. The proof now follows by induction.

Lemma 1.4. Let $G$ be a cocompact, discrete group of isometries of $\mathbb{H}^{2}$. Let $D$ be a Dirichlet region for the action of $G$, and suppose that $D$ has five edges and that exactly two of the angles of $D$ have measure $2 \pi / 3$. The the following conditions hold for every nonnegative integer $n$ :

(i) $B(n)$ is homeomorphic to a ball;

(ii) if $g \in G$ with $|g|=n+1$, then $g D \cap B(n)$ is connected;

(iii) if $g, h \in G$ with $|g|=|h|=n+1$ and $g D \cap h D \neq \varnothing$, then $g D \cap h D$ contains a vertex of $\partial B(n)$; and

(iv) each vertex of $\partial B(n)$ has valence 1 or 2 in $B(n)$.

Proof. As before, we may assume that the measure of an angle of $D$ is $2 \pi$ divided by the valence of the vertex at which it is based. The proof is by 

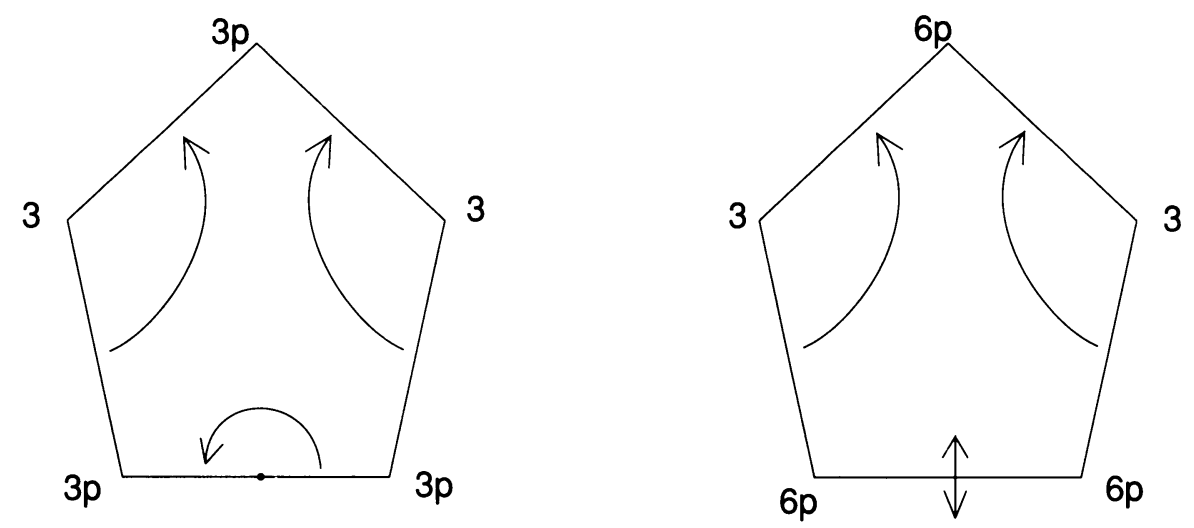

Figure 2. Pentagonal domains with two vertices of valence 3

induction, and uses Lemma 1.1. Since (i)-(iv) are obvious for $n=0$, assume that $n$ is a nonnegative integer and that (i)-(iv) hold for $n$. Let $\theta$ be the minimum measure of an angle of $D$.

Since exactly two of the vertices of $D$ have valence three, it follows from Figure 2 that the other vertices all have valence $3 p$ for some integer $p \geq 2$. Suppose that $g \in G$ with $|g|=n+1$ and $g D \cap B(n)$ not connected, and let $C, r$, and $t$ be chosen as in the proof of Lemma 1.2. Since the two vertices of $D$ with valence 3 are not adjacent, two adjacent vertices in $\partial B(n)$ cannot both have valence 3 in the tessellation. This implies that the sum of the $t-1$ vertices of $C$ in $B(n)-g D$ is at least $(t-1) \pi-\pi / 3$. By the Gauss-Bonnet formula,

$$
\pi\left(\frac{2}{3}-\frac{r}{3}-\frac{2 \theta}{\pi}\right) \geq \operatorname{area}(C) \geq \operatorname{area}(D) \geq \frac{2 \pi}{3} .
$$

Since this is a contradiction, $g D \cap B(n)$ is connected. This proves (ii) for $n+1$.

Now suppose that $g, h \in G$ such that $|g|=|h|=n+1, g D \cap h D \neq \varnothing$, and $g D \cap h D$ does not contain a vertex of $\partial B(n)$. Let $C, r, s$, and $t$ be chosen as in the proof of Lemma 1.2. Then

$\operatorname{area}(C) \leq \pi(r+s+t-2)-\frac{4 \pi}{3}(r+s-2)-(t-1) \pi+\frac{\pi}{3}-3 \theta=\pi\left(-\frac{r}{3}-\frac{s}{3}+2-\frac{3 \theta}{\pi}\right)$,

so $2 \cdot \operatorname{area}(D)>\operatorname{area}(C)$ and hence $C=D$. This is a contradiction, so $g D \cap h D$ contains a vertex of $\partial B(n)$ and (iii) holds for $n+1$. By Lemma 1.1, (i) and (iv) hold for $n+1$ and the proof follows by induction.

Lemma 1.5. Let $G$ be a cocompact, discrete group of isometries of $\mathbb{E}^{2}$ or $\mathbb{H}^{2}$ and let $D$ be a Dirichlet region for the action of $G$ such that $D$ has five edges. Suppose further that exactly three of the vertices of $D$ have valence 3 and that these three vertices are adjacent. The the following conditions hold for every nonnegative integer $n$ :

(i) $B(n)$ is homeomorphic to a ball;

(ii) if $g \in G$ with $|g|=n+1$, then $g D \cap B(n)$ is connected;

(iii) if $g, h \in G$ with $|g|=|h|=n+1$ and $g D \cap h D \neq \varnothing$, then $g D \cap h D$ contains a vertex of $\partial B(n)$; 
(iv) each vertex of $\partial B(n)$ has valence 1 or 2 in $B(n)$; and

(v) there is no edge $e$ in $\partial B(n)$ such that both of the vertices of $\partial e$ have angle measure $4 \pi / 3$ in $B(n)$.

Proof. As before, we may assume that the measure of an angle of $D$ is $2 \pi$ divided by the valence of the vertex at which it is based. The proof is by induction, and uses Lemma 1.1. Since (i)-(v) are obvious for $n=0$, assume that $n$ is a nonnegative integer and that $(\mathrm{i})-(\mathrm{v})$ hold for $\mathrm{n}$. Let $\theta$ be the minimum measure of an angle of $D$.

Suppose that $g \in G$ with $|g|=n+1$ and $g D \cap B(n)$ not connected, and let $C, r$, and $t$ be chosen as in the proof of Lemma 1.2. Suppose $v$ is a vertex in $\partial B(n)$ with angle measure $4 \pi / 3$ in $B(n)$. Then $v$ and both of the vertices adjacent to it, $v_{1}$ and $v_{2}$, in $\partial B(n)$ lie in $h D$ for some $h \in G$. Then by $(\mathrm{v})$ one of the vertices, which we may assume is $v_{1}$, adjacent to $v$ has angle measure $2 \pi / 3$ in $B(n)$ and the other vertex, $v_{2}$, adjacent to $v$ has angle measure at most $\pi$ in $B(n)$. If $v_{2}$ does not have valence 3 in the tessellation $\{g D: g \in G\}$, then the vertex adjacent to $v_{1}$ in $\partial B(n)$ that is not $v$ also does not have valence 3 in the tessellation. Thus the sum of the $t-1$ vertices of $C$ in $B(n)-g D$ is at least $(t-1) \pi-\pi / 3$. If $G$ acts on $\mathbb{H}^{2}$, then by the Gauss-Bonnet formula,

$$
\pi\left(\frac{2}{3}-\frac{r}{3}-\frac{2 \theta}{\pi}\right) \geq \operatorname{area}(C) \geq \operatorname{area}(D) \geq \frac{\pi}{3} .
$$

If $G$ acts on $\mathbb{E}^{2}$, then

$$
\pi(r+t-2) \geq(t-1) \pi-\frac{\pi}{3}+(r-1) \frac{4 \pi}{3}+2 \frac{\pi}{2},
$$

so $-1 / 3 \geq r / 3$. Since both of these are contradictions, $g D \cap B(n)$ is connected and (ii) holds for $n+1$.

Now suppose that $g, h \in G$ such that $|g|=|h|=n+1, g D \cap h D \neq \varnothing$, and $g D \cap h D$ does not contain a vertex of $\partial B(n)$. Let $C, r, s$, and $t$ be chosen as in the proof of Lemma 1.2. If $G$ acts on $\mathbb{H}^{2}$, then

$\operatorname{area}(C) \leq \pi(r+s+t-2)-\frac{4 \pi}{3}(r+s-2)-(t-1) \pi+\frac{\pi}{3}-3 \theta=\pi\left(-\frac{r}{3}-\frac{s}{3}+2-\frac{3 \theta}{\pi}\right)$, so $2 \cdot \operatorname{area}(D)>\operatorname{area}(C)$ and hence $C=D$. If $G$ acts on $\mathbb{E}^{2}$, then

$$
\pi(r+s+t-2) \geq(t-1) \pi-\frac{\pi}{3}+(r+s-2) \frac{4 \pi}{3}+3 \frac{\pi}{2},
$$

so $\frac{1}{2} \geq \frac{r+s}{3}$. These are both contradictions, so $g D \cap h D$ contains a vertex of $\partial B(n)$ and (iii) holds for $n+1$. By Lemma 1.1, (i) and (iv) hold for $B(n+1)$. Now suppose that $e$ is an edge in $\partial B(n+1)$ such that both of the vertices of $\partial e$ have angle measure $4 \pi / 3$ in $B(n+1)$, and let $g \in G$ with $|g|=n+1$ and $e \subset g D$. Then $g D \cap B(n)$ is a union of two edges intersecting in a vertex of valence 3. But then $g D$ has three vertices of valence 3 which are not adjacent, contradicting the hypothesis. This establishes $(v)$ for $n+1$ and the proof follows by induction.

\section{THE RECURSIVE STRUCTURE}

Let $G$ be a cocompact, discrete group of isometries of $\mathbb{E}^{2}$ or $\mathbb{H}^{2}$, and let $D$ be a Dirichlet region for the action of $G$. For the rest of this section, we will 
assume that either $D$ has at least six sides, $D$ has four sides and no vertex of $D$ has valence 3 , or $D$ has five sides and $D$ does not have three vertices that are not adjacent and that each have valence 3.

Our formula for the growth function will be in terms of a linear recursion on the angles of the boundaries of the combinatorial balls. By the previous section, if $v$ is a vertex of a combinatorial ball $B(n)$, then $v$ has valence 1 or 2 in $B(n)$. Thus the angle at $v$ is a translate of one of the angles of $D$ (primitive angles) or is a pair of primitive angles whose intersection is a translate of one of the edge-pairs of $\partial D$. We will define the recursive structure in terms of these primitive angles and edge-pairs.

Let $T$ be the tessellation $\{g D: g \in G\}$. A primitive angle is the equivalence class under $G$ of one of the angles of $D$. The primitive angles correspond exactly to the angles of $D$. Let $a_{1}, \ldots, a_{r}$ be an enumeration of the primitive angles, and let $\mathscr{A}=\left\{a_{1}, \ldots, a_{r}\right\}$. An edge-pair is the equivalence class under the action of $G$ of an edge in $T$ together with one of the vertices in its boundary. Let $p_{1}, \ldots, p_{s}$ be an enumeration of the edge-pairs, and let $\mathscr{P}=\left\{p_{1}, \ldots, p_{s}\right\}$. Then $r$ is the number of sides of $D$, and $s$ is $r$ plus the number of face-pairing elements of $G$ which are reflections. A cycle is the equivalence class under the action of $G$ of a vertex in the tessellation. Let $\mathscr{C}$ be the set of cycles. We will define the following functions:

$$
\begin{aligned}
& a 1: \mathscr{P} \rightarrow \mathscr{A}, \quad a 2: \mathscr{P} \rightarrow \mathscr{A}, \\
& c: \mathscr{A} \cup \mathscr{P} \rightarrow \mathscr{C}, \quad d: \mathscr{P} \rightarrow \mathscr{P}, \\
& l: \mathscr{A} \cup \mathscr{P} \rightarrow \mathbb{N}, \quad m: \mathscr{A} \cup \mathscr{P} \rightarrow \mathbb{N}, \\
& p 1: \mathscr{A} \rightarrow \mathscr{P}, \quad p 2: \mathscr{A} \rightarrow \mathscr{P}, \\
& r: \mathscr{P} \rightarrow\{1,2\} .
\end{aligned}
$$

Given a primitive angle $a$, let $p 1(a)$ and $p 2(a)$ be the two edge-pairs which contain $a$, let $c(a)$ be the cycle at which $a$ is based, let $l(a)$ be the number of cells $g D$ whose boundary contains a fixed representative vertex in the class of $c(a)$, and let $m(a)$ be the number of representatives of the primitive angle a based at a fixed representative vertex in the cycle $c(a)$. Given an edge-pair $p$, let $a 1(p)$ and $a 2(p)$ be the angles adjacent to $p$, let $c(p)$ be the cycle at which $p$ is based, let $l(p)$ be the number of cells $g D$ whose boundary contains a fixed representative vertex in the class of $c(p)$, let $m(p)$ be the number of representatives of the edge-pair $p$ based at a fixed representative vertex in the cycle $c(p)$, let $r(p)=2$ if a representative of $p$ is fixed by a reflection in $G$, and let $r(p)=1$ if a representative of $p$ is not fixed by a reflection of $G$. If $p$ is an edge-pair, then the dual pair $d(p)$ is the edge-pair determined by the same edge but with the opposite vertex. Note that $d$ is an involution on $\mathscr{P}$.

Let $v$ be a vertex in the tessellation. If $v$ is not fixed by a reflection in $G$, then there exist $k, p \in \mathbb{N}$, distinct elements $b_{1}, \ldots, b_{k} \in \mathscr{A}$, and distinct elements $q_{1}, \ldots, q_{k} \in \mathscr{P}$ such that the words of primitive angles and edge-pairs based at $v$, in cyclic order, are $\left(b_{1} \cdots b_{k}\right)^{p}$ and $\left(q_{1} \cdots q_{k}\right)^{p}$. If $v$ is fixed by a reflection in $G$, then there exist $k, p \in \mathbb{N}$, distinct elements $b_{1}, \ldots, b_{k} \in$ $\mathscr{A}$, and distinct elements $q_{1}, \ldots, q_{k+1} \in \mathscr{P}$ such that the words of primitive angles and edge-pairs based at $v$, in cyclic order, are $\left(b_{1} \cdots b_{k} b_{k} \cdots b_{1}\right)^{2 p}$ and $\left(q_{1} q_{2} \cdots q_{k} q_{k+1} q_{k} \cdots q_{2}\right)^{2 p}$ (if $k=1$, these are $\left(b_{1}\right)^{4 p}$ and $\left.\left(q_{1} q_{2}\right)^{2 p}\right)$. In the latter case, either $a 1\left(q_{1}\right)=a 2\left(q_{1}\right)=b_{1}$ and $a 1\left(q_{k+1}\right)=a 2\left(q_{k+1}\right)=b_{k}$ or 

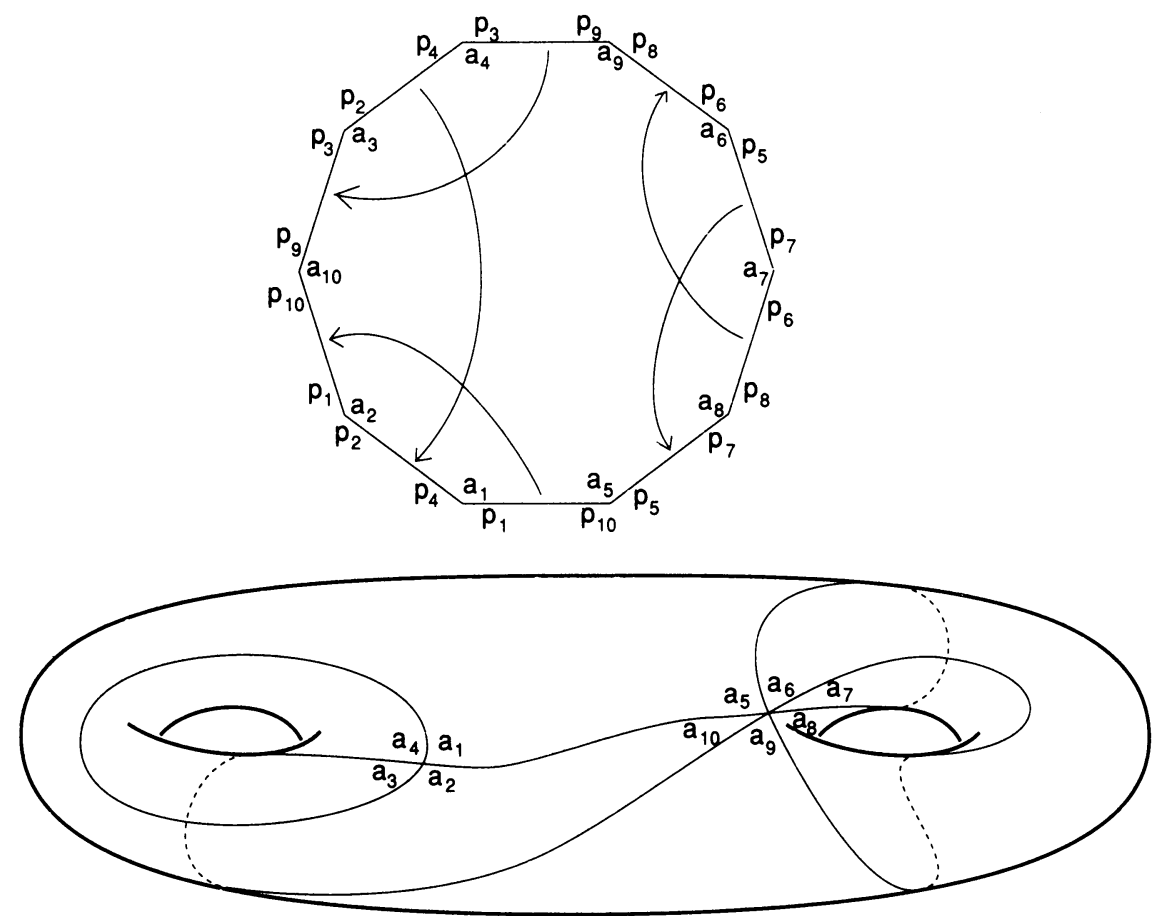

FIGURE 3. An example

$a 1\left(q_{1}\right)=a 2\left(q_{1}\right)=b_{k}$ and $a 1\left(q_{k+1}\right)=a 2\left(q_{k+1}\right)=b_{1}$.

Here is an example to illustrate these functions. In Figure 3 a closed, orientable surface of genus 2 is shown with a spine that corresponds to a 10 -sided Dirichlet domain. Since there are no generators of finite order, $m(a)=m(p)=$ 1 for each primitive angle $a$ and each edge-pair $p$. Since the group is orientable, $r(p)=1$ for each edge-pair $p$. Let $c_{1}$ be the equivalence class of a vertex of valence 4 and let $c_{2}$ be the equivalence class of a vertex of valence 6 . The functions $a 1, a 2, c, d, l, p 1$, and $p 2$ are as follows:

$\begin{array}{cccccccccc}i & a 1\left(p_{i}\right) & a 2\left(p_{i}\right) & c\left(a_{i}\right) & c\left(p_{i}\right) & d\left(p_{i}\right) & l\left(a_{i}\right) & l\left(p_{i}\right) & p 1\left(a_{i}\right) & p 2\left(a_{i}\right) \\ & & & & & & & & & \\ 1 & a_{1} & a_{2} & c_{1} & c_{1} & p_{10} & 4 & 4 & p_{1} & p_{4} \\ 2 & a_{2} & a_{3} & c_{1} & c_{1} & p_{4} & 4 & 4 & p_{2} & p_{1} \\ 3 & a_{3} & a_{4} & c_{1} & c_{1} & p_{9} & 4 & 4 & p_{3} & p_{2} \\ 4 & a_{4} & a_{1} & c_{1} & c_{1} & p_{2} & 4 & 4 & p_{4} & p_{3} \\ 5 & a_{5} & a_{6} & c_{2} & c_{2} & p_{7} & 6 & 6 & p_{5} & p_{10} \\ 6 & a_{6} & a_{7} & c_{2} & c_{2} & p_{8} & 6 & 6 & p_{6} & p_{5} \\ 7 & a_{7} & a_{8} & c_{2} & c_{2} & p_{5} & 6 & 6 & p_{7} & p_{6} \\ 8 & a_{8} & a_{9} & c_{2} & c_{2} & p_{6} & 6 & 6 & p_{8} & p_{7} \\ 9 & a_{9} & a_{10} & c_{2} & c_{2} & p_{3} & 6 & 6 & p_{9} & p_{8} \\ 10 & a_{10} & a_{5} & c_{2} & c_{2} & p_{1} & 6 & 6 & p_{10} & p_{9}\end{array}$

Let $\mathscr{V}$ be the vector space with basis $\mathscr{A} \cup \mathscr{P}$. We will define an inner product on $\mathscr{V}$ by choosing $\mathscr{A} \cup \mathscr{P}$ to be an orthonormal basis. The recursion matrix $A$ is a linear transformation $A: \mathscr{V} \rightarrow \mathscr{V}$. We will write $A$ as a matrix with respect to the basis $\mathscr{A} \cup \mathscr{P}$. 
In order to get closed form expressions for the recursion matrix and its inverse, it will be convenient to define, for a primitive angle $a$ and an edge-pair $p$, the following subsets of $\mathscr{V}$ :

$\operatorname{ACD}(a)$ (angle cycle distinct from $a)$;

$\operatorname{ACF}(a)$ (angle cycle far from $a$ );

$\operatorname{ACF}(p)$ (angle cycle far from $p$ );

$\operatorname{PCD}(p)$ (pair cycle distinct from $p$ );

$\operatorname{PCF}(a)$ (pair cycle far from $a$ ); and

$\operatorname{PCF}(p)$ (pair cycle far from $p$ ).

$$
\begin{aligned}
\operatorname{ACD}(a)= & \left(\sum_{\left\{a_{i}: c\left(a_{i}\right)=c(a)\right\}} m\left(a_{i}\right) a_{i}\right)-a, \\
\operatorname{ACF}(a)= & \left(\sum_{\left\{a_{i}: c\left(a_{i}\right)=c(a)\right\}} m\left(a_{i}\right) a_{i}\right) \\
& +a-a 1(p 1(a))-a 2(p 1(a))-a 1(p 2(a))-a 2(p 2(a)), \\
\operatorname{ACF}(p)= & \left(\sum_{\left\{a_{i}: c\left(a_{i}\right)=c(p)\right\}} m\left(a_{i}\right) a_{i}\right)-a 1(p)-a 2(p), \\
\operatorname{PCD}(p)= & \left(\sum_{\left\{p_{i}: c\left(p_{i}\right)=c(p)\right\}} m\left(p_{i}\right) p_{i}\right)-p, \\
\operatorname{PCF}(a)= & \left(\sum_{\left\{p_{i}: c\left(p_{i}\right)=c(a)\right\}} m\left(p_{i}\right) p_{i}\right)-p 1(a)-p 2(a), \\
\operatorname{PCF}(p)= & \left(\sum_{\left\{p_{i}: c\left(p_{i}\right)=c(p)\right\}} m\left(p_{i}\right) p_{i}\right), \\
& +p-p 1(a 1(p))-p 2(a 1(p))-p 1(a 2(p))-p 2(a 2(p)) .
\end{aligned}
$$

It will also be convenient to have analogues of $\operatorname{PCD}(p), \operatorname{PCF}(a)$, and $\operatorname{PCF}(p)$ that are sets and not subsets of $\mathscr{V}$. Since we need to allow for multipicity greater than 1, we define these in terms of edges emanating from a fixed vertex rather than in terms of equivalence classes of edges. Given a primitive angle $a$, let $\alpha$ be an angle in some $g D, g \in G$, with equivalence class $[\alpha]=a$, and let $v$ be the vertex at which $\alpha$ is based. Let $a c(a)=\{\beta: \beta$ is an angle in $T$ based at $v\}$ and let $p c(a)=\{(e, v): e$ is an edge in $T$ and $v \in \partial E\}$. Then $\operatorname{pcf}(a)=p c(a) \backslash\left\{\left(e_{1}, v\right),\left(e_{2}, v\right)\right\}$, where $e_{1}$ and $e_{2}$ are the two edges whose rays from $v$ make up the angle $\alpha$. Given an edge-pair $p$, let $\rho$ be an edge in some $g D$ and let $v$ be one of the boundary points of $\rho$ such that the equivalence class $[(\rho, v)]=p$. Then

$$
\operatorname{pcd}(p)=\{(e, v): e \text { is an edge in the tessellation and } v \in \partial E\} \backslash\{(\rho, v)\}
$$


and

$\operatorname{pcf}(p)=\{(e, v): e$ is an edge in the tessellation

$$
\text { and } v \in \partial E\} \backslash\left\{(\rho, v),\left(e_{1}, v\right),\left(e_{2}, v\right)\right\},
$$

where $e_{1}$ and $\rho$ lie in $g D$ for some $g \in G$ and $e_{2}$ and $\rho$ lie in $h D$ for some $h \in G$.

Since much of the complication arises when the group $G$ has generators of finite order, in Figure 4 we show a Dirichlet domain with six sides and edgepairing elements of order 2,2 , and 3. For each primitive angle $a, \operatorname{ACD}(a)$, $\operatorname{ACF}(a)$, and $\operatorname{PCF}(a)$ are as follows:

$$
\begin{array}{cccc}
i & \operatorname{ACD}\left(a_{i}\right) & \operatorname{ACF}\left(a_{i}\right) & \operatorname{PCF}\left(a_{i}\right) \\
& & & \\
1 & a_{1}+2 a_{2}+2 a_{3} & 2 a_{2}+a_{3} & p_{2}+2 p_{3}+p_{4} \\
2 & 2 a_{1}+a_{2}+2 a_{3} & 2 a_{1}+a_{3} & p_{1}+p_{3}+2 p_{4} \\
3 & 2 a_{1}+2 a_{2}+a_{3} & a_{1}+a_{2}+a_{3} & p_{1}+p_{2}+p_{3}+p_{4} \\
4 & a_{4}+2 a_{5} & a_{4} & p_{5}+p_{6} \\
5 & 2 a_{4}+a_{5} & a_{5} & p_{5}+p_{6} \\
6 & a_{6} & 0 & p_{7}
\end{array} .
$$

For each edge-pair $p, \operatorname{ACF}(P), \operatorname{PCD}(P)$, and $\operatorname{PCF}(P)$ are as follows:

$$
i \quad \operatorname{ACF}\left(p_{i}\right) \quad \operatorname{PCD}\left(p_{i}\right) \quad \operatorname{PCF}\left(p_{i}\right)
$$

$\begin{array}{cccc}1 & 2 a_{2}+2 a_{3} & p_{2}+2 p_{3}+2 p_{4} & p_{2}+2 p_{3} \\ 2 & 2 a_{1}+2 a_{3} & p_{1}+2 p_{3}+2 p_{4} & p_{1}+2 p_{4} \\ 3 & 2 a_{1}+a_{2}+a_{3} & p_{1}+p_{2}+p_{3}+2 p_{4} & p_{1}+p_{3}+p_{4} \\ 4 & a_{1}+2 a_{2}+a_{3} & p_{1}+p_{2}+2 p_{3}+p_{4} & p_{2}+p_{3}+p_{4} \\ 5 & a_{4}+a_{5} & p_{5}+2 p_{6} & p_{5} \\ 6 & a_{5}+a_{5} & 2 p_{5}+p_{6} & p_{6} \\ 7 & a_{6} & 2 p_{7} & 0\end{array}$
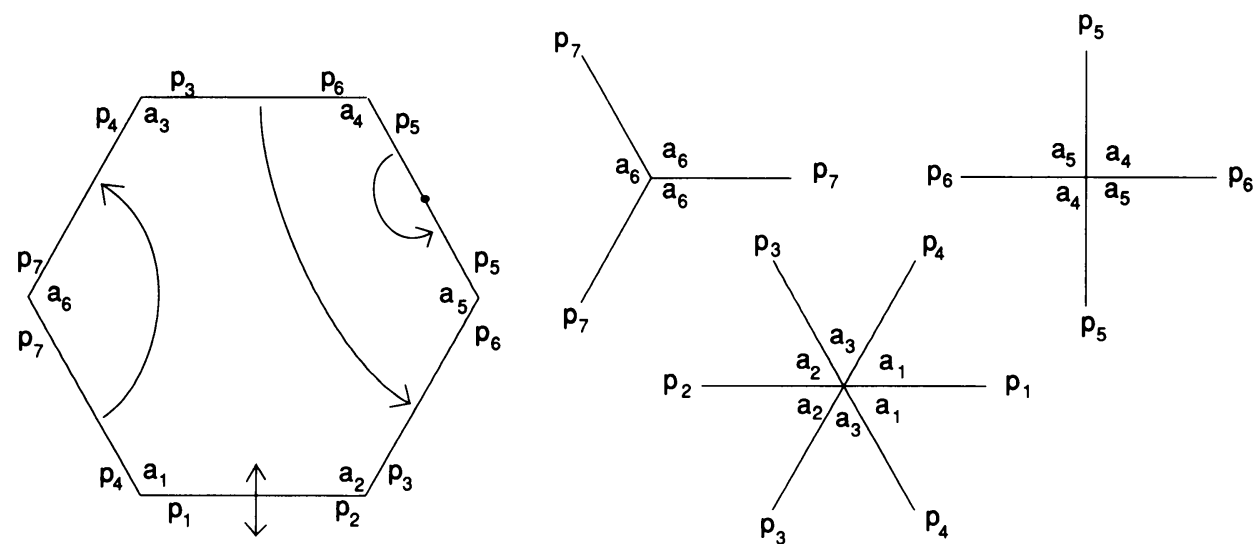

Figure 4. An example with torsion 
The recursion matrix $A$ is defined as follows. To get the $a_{k}$ th column, consider a vertex $v$ in $\partial B(n)$ for some $n$ which has valence 1 and primitive angle $a_{k}$. There are $l\left(a_{k}\right)-1 \quad g \in G$ with $|g|=n+1$ and $v \in g D$. Since the ones attached to $B(n)$ along an edge will have two vertices of $\partial B(n)$ in their boundary, $a_{k}$ counts them with multiplicity $1 / 2$. Since $D$ has one of each primitive angle, this gives $l\left(a_{k}\right)-2$ of each primitive angle as a first approximation for the primitive angles in $\partial B(n+1)$ coming from $a_{k}$. However, we need to subtract the primitive angles besides $a_{k}$ which are based in the cycle of $v$. Finally, for each edge-pair based at $v$ which is not in $B(n)$ we need to add the dual edge-pair and subtract the two primitive angles of the dual edge pair. So the $a_{k}$ th column of $A$ is

$$
\sum_{a_{i} \in \mathscr{A}}\left(l\left(a_{k}\right)-2\right) a_{i}-\operatorname{ACD}\left(a_{k}\right)+\sum_{p \in \operatorname{pcf}\left(a_{k}\right)} d([p])-a 1(d([p]))-a 2(d([p])) .
$$

The column corresponding to an edge-pair $p_{k}$ is similar. This time there are $l\left(p_{k}\right)-3$ of each primitive angle, minus the primitive angles based at $v$ which are not in $B(n)$, plus the dual edge-pairs of the edge-pairs based at $v$ which are not in $B(n)$, minus the primitive angles in those dual edge-pairs. So the $p_{k}$ th column of $A$ is

$$
\sum_{a_{i} \in \mathscr{A}}\left(l\left(p_{k}\right)-3\right) a_{i}-\operatorname{ACF}\left(p_{k}\right)+\sum_{p \in \operatorname{pcf}\left(p_{k}\right)} d([p])-a 1(d([p]))-a 2(d([p]))
$$

In the example from Figure 3,

$$
A=\left[\begin{array}{llllllllllllllllllll}
1 & 1 & 1 & 0 & 4 & 3 & 3 & 3 & 3 & 4 & 1 & 0 & 0 & 0 & 3 & 2 & 2 & 2 & 3 & 3 \\
1 & 1 & 0 & 1 & 4 & 3 & 3 & 3 & 3 & 4 & 1 & 0 & 0 & 0 & 3 & 2 & 2 & 2 & 3 & 3 \\
1 & 0 & 1 & 1 & 3 & 3 & 3 & 3 & 4 & 4 & 0 & 0 & 1 & 0 & 2 & 2 & 2 & 3 & 3 & 3 \\
0 & 1 & 1 & 1 & 3 & 3 & 3 & 3 & 4 & 4 & 0 & 0 & 1 & 0 & 2 & 2 & 2 & 3 & 3 & 3 \\
2 & 2 & 1 & 1 & 3 & 2 & 3 & 3 & 2 & 2 & 1 & 1 & 0 & 1 & 2 & 2 & 2 & 2 & 1 & 2 \\
2 & 2 & 2 & 2 & 1 & 2 & 2 & 3 & 2 & 1 & 1 & 1 & 1 & 1 & 1 & 2 & 2 & 2 & 1 & 0 \\
2 & 2 & 2 & 2 & 2 & 2 & 2 & 2 & 2 & 1 & 1 & 1 & 1 & 1 & 1 & 2 & 2 & 1 & 1 & 1 \\
2 & 2 & 2 & 2 & 2 & 3 & 2 & 2 & 1 & 1 & 1 & 1 & 1 & 1 & 2 & 2 & 2 & 1 & 0 & 1 \\
1 & 1 & 2 & 2 & 2 & 3 & 3 & 2 & 3 & 2 & 0 & 1 & 1 & 1 & 2 & 2 & 2 & 2 & 2 & 1 \\
1 & 1 & 1 & 1 & 3 & 3 & 3 & 3 & 3 & 4 & 0 & 1 & 0 & 1 & 2 & 2 & 2 & 2 & 3 & 3 \\
0 & 0 & 0 & 0 & 0 & 1 & 1 & 1 & 1 & 0 & 0 & 0 & 0 & 0 & 0 & 1 & 1 & 1 & 0 & 0 \\
0 & 1 & 1 & 0 & 0 & 0 & 0 & 0 & 0 & 0 & 0 & 1 & 0 & 0 & 0 & 0 & 0 & 0 & 0 & 0 \\
0 & 0 & 0 & 0 & 1 & 1 & 1 & 1 & 0 & 0 & 0 & 0 & 0 & 0 & 1 & 1 & 1 & 0 & 0 & 0 \\
1 & 0 & 0 & 1 & 0 & 0 & 0 & 0 & 0 & 0 & 0 & 0 & 0 & 1 & 0 & 0 & 0 & 0 & 0 & 0 \\
0 & 0 & 0 & 0 & 1 & 1 & 0 & 0 & 1 & 1 & 0 & 0 & 0 & 0 & 1 & 0 & 0 & 0 & 1 & 1 \\
0 & 0 & 0 & 0 & 1 & 1 & 1 & 0 & 0 & 1 & 0 & 0 & 0 & 0 & 1 & 1 & 0 & 0 & 0 & 1 \\
0 & 0 & 0 & 0 & 0 & 0 & 1 & 1 & 1 & 1 & 0 & 0 & 0 & 0 & 0 & 0 & 1 & 1 & 1 & 0 \\
0 & 0 & 0 & 0 & 1 & 0 & 0 & 1 & 1 & 1 & 0 & 0 & 0 & 0 & 0 & 0 & 0 & 1 & 1 & 1 \\
1 & 1 & 0 & 0 & 0 & 0 & 0 & 0 & 0 & 0 & 1 & 0 & 0 & 0 & 0 & 0 & 0 & 0 & 0 & 0 \\
0 & 0 & 1 & 1 & 0 & 0 & 0 & 0 & 0 & 0 & 0 & 0 & 1 & 0 & 0 & 0 & 0 & 0 & 0 & 0
\end{array}\right] .
$$


In the example from Figure 4,

$$
A=\left[\begin{array}{ccccccccccccc}
1 & 2 & 0 & 2 & 2 & 0 & 1 & 1 & 1 & 0 & 1 & 1 & 0 \\
2 & 1 & 0 & 1 & 1 & 1 & 1 & 1 & 0 & 1 & 1 & 0 & 0 \\
2 & 2 & 3 & 1 & 1 & 0 & 1 & 1 & 2 & 2 & 1 & 0 & 0 \\
2 & 3 & 3 & 0 & -1 & 1 & 1 & 3 & 2 & 2 & -1 & 0 & 0 \\
2 & 3 & 3 & -1 & 0 & 1 & 1 & 3 & 2 & 2 & -1 & 0 & 0 \\
2 & 0 & 2 & 2 & 2 & -1 & 3 & -1 & 1 & 1 & 1 & 1 & -1 \\
1 & 0 & 1 & 0 & 0 & 0 & 1 & 0 & 0 & 1 & 0 & 0 & 0 \\
0 & 1 & 1 & 0 & 0 & 0 & 0 & 1 & 1 & 0 & 0 & 0 & 0 \\
0 & 0 & 0 & 1 & 1 & 0 & 0 & 0 & 0 & 0 & 0 & 1 & 0 \\
0 & 0 & 0 & 0 & 0 & 1 & 0 & 0 & 0 & 0 & 0 & 0 & 0 \\
0 & 0 & 0 & 1 & 1 & 0 & 0 & 0 & 0 & 0 & 1 & 0 & 0 \\
2 & 1 & 1 & 0 & 0 & 0 & 2 & 0 & 1 & 1 & 0 & 0 & 0 \\
1 & 2 & 1 & 0 & 0 & 0 & 0 & 2 & 1 & 1 & 0 & 0 & 0
\end{array}\right] .
$$

Let $U$ be the row vector with $a_{j}$ entry 0 and $p_{j}$ entry 1 , let $W$ be the column vector with $a_{j}$ entry 1 and $p_{j}$ entry 0 , and let $V=A W$. It follows by induction that, for every nonnegative integer $n, A^{n} W$ has $a_{i}$ entry the number of times the primitive angle $a_{i}$ appears in $\partial B(n)$ and $p_{i}$ entry the number of times the edge-pair $p_{i}$ is the interior edge-pair in a pair of primitive angles in $\partial B(n)$. If $g \in G$ with $|g|=n, n \geq 1$, then $g D \cap B(n)$ contains exactly two vertices with valence 2 in $B(n)$. Since each vertex in $\partial B(n)$ with valence 2 is in the boundary of exactly two translates of $D$ by elements of word norm $n$, if $n \geq 1$ then the $n$th coefficient of the growth series for $G$ with full geometric generating set $\Sigma$ corresponding to $D$ is $s_{n}=U A^{n} W$.

Lemma 2.1. With the notation as above, the growth function is $f(z)=1+$ $U(I-z A)^{-1} z V$.

Proof. The growth series

$$
\begin{aligned}
g(z) & =1+s_{1} z+s_{2} z^{2}+\cdots+s_{n} z^{n}+\cdots \\
& =1+U V z+U A V z^{2}+\cdots+U A^{n} V z^{n}+\cdots \\
& =1+U\left(I+z A+\cdots+z^{n-1} A^{n-1}+\cdots\right) z V
\end{aligned}
$$

which is the Maclaurin series of the function $1+U(I-z A)^{-1} z V$.

Let $B$ be the matrix with $a_{k}$ th column $\operatorname{ACF}\left(a_{k}\right)-\operatorname{PCF}\left(a_{k}\right)$ and with $p_{k}$ th column

$$
\begin{aligned}
\left(\sum_{a_{i} \in \mathscr{A}}(3-\right. & \left.\left.l\left(a_{i}\right)\right) a_{i}\right)+\operatorname{ACF}\left(a 1\left(p_{k}\right)\right)+\operatorname{ACF}\left(a 2\left(p_{k}\right)\right)+\operatorname{ACF}\left(d\left(p_{k}\right)\right) \\
& +\left(\sum_{p_{i} \in \mathscr{P}} \frac{l\left(p_{i}\right)-2}{r\left(p_{i}\right)} p_{i}\right)-\operatorname{PCD}\left(d\left(p_{k}\right)\right)-\operatorname{PCF}\left(a 1\left(p_{k}\right)\right)-\operatorname{PCF}\left(a 2\left(p_{k}\right)\right) .
\end{aligned}
$$

We will show that $B=A^{-1}$. 
In the example from Figure $3, B$ is the matrix

$$
\left[\begin{array}{cccccccccccccccccccc}
0 & 0 & 1 & 0 & 0 & 0 & 0 & 0 & 0 & 0 & -1 & 0 & 0 & 0 & -1 & -1 & -1 & -1 & 0 & -1 \\
0 & 0 & 0 & 1 & 0 & 0 & 0 & 0 & 0 & 0 & -1 & 0 & 0 & 0 & -1 & -1 & -1 & -1 & 0 & -1 \\
1 & 0 & 0 & 0 & 0 & 0 & 0 & 0 & 0 & 0 & 0 & 0 & -1 & 0 & -1 & -1 & -1 & -1 & -1 & 0 \\
0 & 1 & 0 & 0 & 0 & 0 & 0 & 0 & 0 & 0 & 0 & 0 & -1 & 0 & -1 & -1 & -1 & -1 & -1 & 0 \\
0 & 0 & 0 & 0 & 0 & 0 & 1 & 1 & 1 & 0 & -3 & -3 & -2 & -3 & -2 & -1 & -1 & 0 & -2 & -3 \\
0 & 0 & 0 & 0 & 0 & 0 & 0 & 1 & 1 & 1 & -2 & -3 & -2 & -3 & -2 & -2 & -2 & -1 & -1 & -2 \\
0 & 0 & 0 & 0 & 1 & 0 & 0 & 0 & 1 & 1 & -2 & -3 & -2 & -3 & -2 & -2 & -2 & -2 & -1 & -1 \\
0 & 0 & 0 & 0 & 1 & 1 & 0 & 0 & 0 & 1 & -2 & -3 & -2 & -3 & -1 & -2 & -2 & -2 & -2 & -1 \\
0 & 0 & 0 & 0 & 1 & 1 & 1 & 0 & 0 & 0 & -2 & -3 & -3 & -3 & 0 & -1 & -1 & -2 & -3 & -2 \\
0 & 0 & 0 & 0 & 0 & 1 & 1 & 1 & 0 & 0 & -3 & -3 & -3 & -3 & -1 & 0 & 0 & -1 & -3 & -3 \\
0 & 0 & -1 & -1 & 0 & 0 & 0 & 0 & 0 & 0 & 2 & 0 & 0 & 0 & 2 & 2 & 2 & 2 & 1 & 2 \\
-1 & 0 & 0 & -1 & 0 & 0 & 0 & 0 & 0 & 0 & 1 & 1 & 1 & 0 & 2 & 2 & 2 & 2 & 1 & 1 \\
-1 & -1 & 0 & 0 & 0 & 0 & 0 & 0 & 0 & 0 & 0 & 0 & 2 & 0 & 2 & 2 & 2 & 2 & 2 & 1 \\
0 & -1 & -1 & 0 & 0 & 0 & 0 & 0 & 0 & 0 & 1 & 0 & 1 & 1 & 2 & 2 & 2 & 2 & 1 & 1 \\
0 & 0 & 0 & 0 & 0 & 0 & -1 & -1 & -1 & -1 & 3 & 4 & 3 & 4 & 3 & 2 & 2 & 1 & 2 & 3 \\
0 & 0 & 0 & 0 & -1 & 0 & 0 & -1 & -1 & -1 & 3 & 4 & 3 & 4 & 2 & 3 & 2 & 2 & 2 & 2 \\
0 & 0 & 0 & 0 & -1 & -1 & 0 & 0 & -1 & -1 & 3 & 4 & 3 & 4 & 2 & 2 & 3 & 2 & 2 & 2 \\
0 & 0 & 0 & 0 & -1 & -1 & -1 & 0 & 0 & -1 & 3 & 4 & 3 & 4 & 1 & 2 & 2 & 3 & 3 & 2 \\
0 & 0 & 0 & 0 & -1 & -1 & -1 & -1 & 0 & 0 & 3 & 4 & 4 & 4 & 1 & 1 & 1 & 2 & 4 & 3 \\
0 & 0 & 0 & 0 & 0 & -1 & -1 & -1 & -1 & 0 & 4 & 4 & 3 & 4 & 2 & 1 & 1 & 1 & 3 & 4
\end{array}\right] .
$$

In the example from Figure 4 ,

$$
B=\left[\begin{array}{ccccccccccccc}
0 & 2 & 1 & 0 & 0 & 0 & -1 & 1 & 0 & -2 & -3 & -1 & -2 \\
2 & 0 & 1 & 0 & 0 & 0 & 1 & -1 & -2 & 0 & -3 & -2 & -1 \\
1 & 1 & 1 & 0 & 0 & 0 & 1 & 1 & -1 & -1 & -3 & -2 & -2 \\
0 & 0 & 0 & 1 & 0 & 0 & -1 & -1 & 0 & -1 & 1 & 0 & -1 \\
0 & 0 & 0 & 0 & 1 & 0 & -1 & -1 & 0 & -1 & 1 & 0 & -1 \\
0 & 0 & 0 & 0 & 0 & 0 & 0 & 0 & 0 & 1 & 0 & 0 & 0 \\
0 & -1 & -1 & 0 & 0 & 0 & 1 & 0 & 0 & 1 & 2 & 1 & 1 \\
-1 & 0 & -1 & 0 & 0 & 0 & 0 & 1 & 1 & 0 & 2 & 1 & 1 \\
-2 & -1 & -1 & 0 & 0 & 0 & -2 & 0 & 2 & 1 & 4 & 3 & 2 \\
-1 & -2 & -1 & 0 & 0 & 0 & 0 & -2 & 1 & 2 & 4 & 2 & 3 \\
0 & 0 & 0 & -1 & -1 & 0 & 2 & 2 & 0 & 2 & -1 & 0 & 2 \\
0 & 0 & 0 & -1 & -1 & 0 & 2 & 2 & 1 & 2 & -2 & 0 & 2 \\
0 & 0 & 0 & 0 & 0 & -1 & 1 & 1 & 1 & -1 & 1 & 1 & -1
\end{array}\right] .
$$

It will be convenient to have expressions for the rows of $A$ and the rows of $B$. The main difficulty in doing this arises when at least one of the face-pairing elements of $D$ is a reflection. Let $c$ be a cycle with representative element a vertex $v$ of $D$ which is fixed by a reflection in $G$. If $a$ is a primitive angle with cycle $c(a)=c$, then the multiplicity $m(a)=2 k$ for some positive integer $k$. If $p$ is an edge-pair with $c(p)=c$, then $m(p)=2 k$ if $p$ does not have a representative edge which is fixed by a reflection in $G$ and $m(p)=k$ if $p$ does have a representative edge which is fixed by an element in $G$. Given a primitive angle $a_{j}$, let

$$
\operatorname{PCF}^{\prime}\left(a_{j}\right)=\sum_{p \in \operatorname{pcf}\left(a_{j}\right)} r([p])[p] .
$$

Given a edge-pair $p_{j}$, let

$$
\operatorname{PCF}^{\prime}\left(p_{j}\right)=\sum_{p \in \operatorname{pcf}\left(p_{j}\right)} r([p])[p] \text { and } \operatorname{PCD}^{\prime}\left(p_{j}\right)=\sum_{p \in \operatorname{pcd}\left(p_{j}\right)} r([p])[p] \text {. }
$$


The following table gives the correspondence between entries in the rows and entries in the columns.

$$
\begin{aligned}
& \text { COLUMNS } \\
& \text { ROWS } \\
& a_{k} \mapsto \sum_{a_{i} \in \mathscr{A}}\left(l\left(a_{k}\right)-2\right) a_{i} \\
& a_{j} \mapsto \sum_{a_{i} \in \mathscr{A}}\left(l\left(a_{i}\right)-2\right) a_{i} \\
& a_{k} \mapsto \operatorname{ACD}\left(a_{k}\right) \\
& a_{j} \mapsto \operatorname{ACD}\left(a_{j}\right) \\
& a_{k} \mapsto \sum_{p \in \operatorname{pcf}\left(a_{k}\right)} a 1(d([p]))+a 2(d([p])) \\
& a_{j} \mapsto \operatorname{ACF}\left(d\left(p 1\left(a_{j}\right)\right)\right)+\operatorname{ACF}\left(d\left(p 2\left(a_{j}\right)\right)\right) \\
& a_{k} \mapsto \operatorname{ACF}\left(a_{k}\right) \\
& a_{j} \mapsto \operatorname{ACF}\left(a_{j}\right) \\
& a_{k} \mapsto \sum_{p \in \operatorname{pcf}\left(a_{k}\right)} d([p]) \\
& p_{j} \mapsto \frac{\operatorname{ACF}\left(d\left(p_{j}\right)\right)}{r\left(d\left(p_{j}\right)\right)} \\
& a_{k} \mapsto \operatorname{PCF}\left(a_{k}\right) \\
& p_{j} \mapsto \frac{\operatorname{ACF}\left(p_{j}\right)}{r\left(p_{j}\right)} \\
& p_{k} \mapsto \sum_{a_{i} \in \mathscr{A}}\left(l\left(p_{k}\right)-3\right) a_{i} \\
& a_{j} \mapsto \sum_{p_{i} \in \mathscr{P}}\left(l\left(p_{i}\right)-3\right) p_{i} \\
& p_{k} \mapsto \sum_{a_{i} \in \mathscr{A}}\left(3-l\left(a_{i}\right)\right) a_{i} \\
& a_{j} \mapsto \sum_{p_{i} \in \mathscr{P}}\left(3-l\left(a_{j}\right)\right) p_{i} \\
& p_{k} \mapsto \operatorname{ACF}\left(p_{k}\right) \\
& a_{j} \mapsto \operatorname{PCF}^{\prime}\left(a_{j}\right) \\
& p_{k} \mapsto \operatorname{ACF}\left(d\left(p_{k}\right)\right) \\
& a_{j} \mapsto \sum_{p \in \operatorname{pcf}\left(a_{j}\right)} r([p]) d([p]) \\
& p_{k} \mapsto \sum_{p \in \operatorname{pcf}\left(p_{k}\right)} a 1(d([p]))+a 2(d([p])) \\
& a_{j} \mapsto \operatorname{PCF}^{\prime}\left(d\left(p 1\left(a_{j}\right)\right)\right)+\operatorname{PCF}^{\prime}\left(d\left(p 2\left(a_{j}\right)\right)\right) \\
& p_{k} \mapsto \operatorname{ACF}\left(a 1\left(p_{k}\right)\right)+\operatorname{ACF}\left(a 2\left(p_{k}\right)\right) \\
& a_{j} \mapsto \operatorname{PCF}^{\prime}\left(p 1\left(a_{j}\right)\right)+\operatorname{PCF}^{\prime}\left(p 2\left(a_{j}\right)\right) \\
& p_{k} \mapsto \sum_{p_{i} \in \mathscr{P}} \frac{l\left(p_{i}\right)-2}{r\left(p_{i}\right)} p_{i} \\
& p_{j} \mapsto \sum_{p_{i} \in \mathscr{P}} \frac{l\left(p_{j}\right)-2}{r\left(p_{j}\right)} p_{i} \\
& p_{k} \mapsto \sum_{p \in \operatorname{pcf}\left(p_{k}\right)} d([p]) \\
& p_{j} \mapsto \frac{\operatorname{PCF}^{\prime}\left(d\left(p_{j}\right)\right)}{r\left(d\left(p_{j}\right)\right)} \\
& p_{k} \mapsto \operatorname{PCF}\left(a 1\left(p_{k}\right)\right)+\operatorname{PCF}\left(a 2\left(p_{k}\right)\right) \\
& p_{j} \mapsto \frac{\operatorname{PCF}^{\prime}\left(a 1\left(p_{j}\right)\right)}{r\left(p_{j}\right)}+\frac{\operatorname{PCF}^{\prime}\left(a 2\left(p_{j}\right)\right)}{r\left(p_{j}\right)} \\
& p_{k} \mapsto \operatorname{PCD}\left(d\left(p_{k}\right)\right) \\
& p_{j} \mapsto \sum_{p \in \operatorname{pcd}\left(p_{j}\right)} \frac{r([p]) d([p])}{r\left(p_{j}\right)}
\end{aligned}
$$

The matrix $A$ has $a_{j}$ th row

$$
\begin{aligned}
& \left(\sum_{a_{i} \in \mathscr{A}}\left(l\left(a_{i}\right)-2\right) a_{i}\right)-\operatorname{ACD}\left(a_{j}\right)-\operatorname{ACF}\left(d\left(p 1\left(a_{j}\right)\right)\right)-\operatorname{ACF}\left(d\left(p 2\left(a_{j}\right)\right)\right) \\
& \quad+\left(\sum_{p_{i} \in \mathscr{P}}\left(l\left(p_{i}\right)-3\right) p_{i}\right)-\operatorname{PCF}^{\prime}\left(a_{j}\right)-\operatorname{PCF}^{\prime}\left(d\left(p 1\left(a_{j}\right)\right)\right)-\operatorname{PCF}^{\prime}\left(d\left(p 2\left(a_{j}\right)\right)\right)
\end{aligned}
$$

and has $p_{j}$ th row

$$
\frac{\operatorname{ACF}\left(d\left(p_{j}\right)\right)}{r\left(d\left(p_{j}\right)\right)}+\frac{\operatorname{PCF}^{\prime}\left(d\left(p_{j}\right)\right)}{r\left(d\left(p_{j}\right)\right)} .
$$

The matrix $B$ has $a_{j}$ th row

$$
\operatorname{ACF}\left(a_{j}\right)+\sum_{p_{i} \in \mathscr{P}}\left(3-l\left(a_{j}\right)\right) p_{i}+\operatorname{PCF}^{\prime}\left(p 1\left(a_{j}\right)\right)+\operatorname{PCF}^{\prime}\left(p 2\left(a_{j}\right)\right)+\sum_{p \in \operatorname{pcf}\left(a_{j}\right)} r([p]) d([p])
$$


and has $p_{j}$ th row

$$
\begin{aligned}
& -\frac{\operatorname{ACF}\left(p_{j}\right)}{\left.r\left(p_{j}\right)\right)}+\sum_{p_{i} \in \mathscr{P}} \frac{l\left(p_{j}\right)-2}{r\left(p_{j}\right)} p_{i} \\
& \quad-\sum_{p \in \operatorname{pcd}\left(p_{j}\right)} \frac{r([p]) d([p])}{r\left(p_{j}\right)}-\frac{\operatorname{PCF}^{\prime}\left(a 1\left(p_{j}\right)\right)}{r\left(p_{j}\right)}-\frac{\operatorname{PCF}^{\prime}\left(a 2\left(p_{j}\right)\right)}{r\left(p_{j}\right)}
\end{aligned}
$$

Lemma 2.2. Let $a \in \mathscr{A}$ and let $p \in \mathscr{P}$. Then

$$
\operatorname{ACF}(p) \cdot \operatorname{ACF}(a)-\operatorname{PCF}^{\prime}(p) \cdot \operatorname{PCF}(a)=0
$$

and

$$
\operatorname{ACF}(p) \cdot \operatorname{ACD}(a)-\operatorname{PCD}(p) \cdot \operatorname{PCF}^{\prime}(a)=0 .
$$

Proof. The lemma is obvious if $c(a) \neq c(p)$, so suppose that $c$ is a cycle such that $c(a)=c=c(p)$. Let

$$
\mathrm{AC}(c)=\sum_{\{b \in \mathscr{A}: c(b)=c\}} m(b) b,
$$

let

$$
\operatorname{PC}(c)=\sum_{\{q \in \mathscr{P}: c(q)=c\}} m(q) q
$$

and let

$$
\mathrm{PC}^{\prime}(c)=\sum_{\{q \in \mathscr{P}: c(q)=c\}} r(q) m(q) q
$$

Then

$$
\mathrm{AC}(c) \cdot \mathrm{AC}(c)=m(a)^{2} \cdot \#\{b \in \mathscr{A}: c(b)=c\}
$$

If no edge-pair $q$ with $c(q)=c$ has a representative fixed by a reflection in $G$, then $\mathrm{PC}(c)=\mathrm{PC}^{\prime}(c)$ and

$$
\mathrm{PC}^{\prime}(c) \cdot \mathrm{PC}(c)=m(p)^{2} \cdot \#\{q \in \mathscr{P}: c(q)=c\}=\mathrm{AC}(c) \cdot \mathrm{AC}(c) .
$$

Otherwise,

$$
\begin{aligned}
\mathrm{PC}^{\prime}(c) \cdot \operatorname{PC}(c) & =m(a)^{2}(\#\{b \in \mathscr{A}: c(b)=c\}-1)+2 \frac{m(a)}{2} 2 \frac{m(a)}{2} \\
& =\mathrm{AC}(c) \cdot \mathrm{AC}(c) .
\end{aligned}
$$

First suppose that no edge-pair $q$ with $c(q)=c$ has a representative fixed by a nontrivial element of $G$. Let

$$
t=\#\{b \in \mathscr{A}: c(b)=c\}=\#\{q \in \mathscr{P}: c(q)=c\},
$$

let $b_{1}, \ldots, b_{t}$ be an enumeration of the elements of $\{b \in \mathscr{A}: c(b)=c\}$, and let $q_{1}, \ldots, q_{t}$ be an enumeration of the elements of $\{q \in \mathscr{P}: c(q)=c\}$, chosen so that $p 1\left(b_{i}\right)=q_{i}, p 2\left(b_{i}\right)=q_{i+1}$ if $i<t$, and $p 2\left(b_{t}\right)=q_{1}$. Then we can define the functions $a 1$ and $a 2$ by $a 1\left(q_{1}\right)=b_{t}, a 1\left(q_{i}\right)=b_{i-1}$ if $i>1$, and $a 2\left(q_{i}\right)=b_{i}$. Given an integer $i \in\{1, \ldots, t\}$, define $i+, i-\in\{1, \ldots, t\}$ by $1-=t, i-=i-1$ if $i>1, t+=1$, and $i+=i+1$ if $i<t$. Define $i$ and $j$ by $a=b_{i}$ and $p=q_{j}$. Since

$$
\mathrm{AC}(p) \cdot \mathrm{AC}(a)=\mathrm{PC}^{\prime}(p) \cdot \mathrm{PC}(a)=\mathrm{PC}(p) \cdot \operatorname{PC}^{\prime}(a),
$$


the lemma reduces to showing that $\left(b_{j}+b_{j-}\right) \cdot\left(b_{i-}+b_{i}+b_{i+}\right)=\left(q_{j-}+q_{j}+\right.$ $\left.q_{j+}\right) \cdot\left(q_{i}+q_{i+}\right)$ and $\left(b_{j}+b_{j-}\right) \cdot b_{i}=q_{j} \cdot\left(q_{i}+q_{i+}\right)$.

$$
\begin{aligned}
\left(b_{j}+b_{j-}\right) \cdot\left(b_{i-}+b_{i}+b_{i+}\right) & =\delta_{j, i-}+\delta_{j, i}+\delta_{j, i+}+\delta_{j-, i-}+\delta_{j-, i}+\delta_{j-, i+} \\
& =\delta_{j+, i}+\delta_{j, i}+\delta_{j, i+}+\delta_{j+, i+}+\delta_{j-, i}+\delta_{j-, i+} \\
& =\left(q_{j-}+q_{j}+q_{j+}\right) \cdot\left(q_{i}+q_{i+}\right)
\end{aligned}
$$

and

$$
\left(b_{j}+b_{j-}\right) \cdot b_{i}=\delta_{j, i}+\delta_{j-, i}=\delta_{j, i}+\delta_{j, i+}=q_{j} \cdot\left(q_{i}+q_{i+}\right) .
$$

Now suppose that there is an edge-pair $q$ with $c(q)=c$ which has a representative fixed by a nontrivial element of $G$. Then exactly two edge-pairs $q$ with $c(q)=c$ have representatives fixed by a nontrivial element of $G$. Let $t=\#\{b \in \mathscr{A}: c(b)=c\}$, let $b_{1}, \ldots, b_{t}$ be an enumeration of the elements of $\{b \in \mathscr{A}: c(b)=c\}$, and let $q_{1}, \ldots, q_{t+1}$ be an enumeration of the elements of $\{q \in \mathscr{P}: c(q)=c\}$, chosen so that $p 1\left(b_{i}\right)=q_{i}, p 2\left(b_{i}\right)=q_{i+1}$, and $r\left(q_{1}\right)=r\left(q_{t+1}\right)=2$ (so $r\left(q_{i}\right)=1$ if $\left.1<i<t+1\right)$. Then we can define the functions $a 1$ and $a 2$ by $a 1\left(q_{1}\right)=b_{1}, a 1\left(q_{i}\right)=b_{i-1}$ if $i>1, a 2\left(q_{i}\right)=b_{i}$ if $i \leq t$, and $a 2\left(q_{t+1}\right)=b_{t}$. Given an integer $i \in\{1, \ldots, t\}$, define $i+, i-\in\{1, \ldots, t\}$ by $1-=1, i-=i-1$ if $i>1, t+=t$, and $i+=i+1$ if $i<t$. Given an integer $j \in\{1, \ldots, t+1\}$, define $j \oplus, j \ominus \in\{1, \ldots, t+1\}$ by $1 \ominus=2$, $j \ominus=j-1$ if $j>1, j \oplus=j+1$ if $j \leq t$, and $(t+1) \oplus=t$. Define $i$ and $j$ by $a=b_{i}$ and $p=q_{j}$. By symmetry, we can assume that $1 \leq j \leq(t+2) / 2$. Since (1) holds, the lemma reduces to showing that

$$
\left(b_{j}+b_{j-}\right) \cdot\left(b_{i-}+b_{i}+b_{i+}\right)=\left(r\left(q_{j \ominus}\right) q_{j \ominus}+r\left(q_{j}\right) q_{j}+r\left(q_{j \oplus}\right) q_{j \oplus}\right) \cdot\left(q_{i}+q_{i \oplus}\right)
$$

and

$$
\left(b_{j}+b_{j-}\right) \cdot b_{i}=q_{j} \cdot\left(r\left(q_{i}\right) q_{i}+r\left(q_{i \oplus}\right) q_{i \oplus}\right) .
$$

Both equations are clear if $t=1$, so assume that $t \geq 2$. If $j=1$, then the equations are

$$
2 b_{1} \cdot\left(b_{i-}+b_{i}+b_{i+}\right)=\left(2 q_{1}+2 q_{2}\right) \cdot\left(q_{i}+q_{i \oplus}\right)
$$

and

$$
2 b_{1} \cdot b_{i}=q_{1} \cdot\left(r\left(q_{i}\right) q_{i}+r\left(q_{i \oplus}\right) q_{i \oplus}\right),
$$

which are easily checked by examining the cases $i=1, i=2$, and $i \geq 3$. If $j=2$, the equations are

$$
\left(b_{2}+b_{1}\right) \cdot\left(b_{i-}+b_{i}+b_{i+}\right)=\left(2 q_{1}+q_{2}+r\left(q_{3}\right) q_{3}\right) \cdot\left(q_{i}+q_{i \oplus}\right)
$$

and

$$
\left(b_{2}+b_{1}\right) \cdot b_{i}=q_{2} \cdot\left(r\left(q_{i}\right) q_{i}+r\left(q_{i \oplus}\right) q_{i \oplus}\right)
$$

which again follow by checking the possibilities for $i$. If $3 \leq j \leq(t+2) / 2$, the equations are

$$
\left(b_{j}+b_{j-}\right) \cdot\left(b_{i-}+b_{i}+b_{i+}\right)=\left(q_{j-}+q_{j}+q_{j+}\right) \cdot\left(q_{i}+q_{i \oplus}\right)
$$

and

$$
\left(b_{j}+b_{-j}\right) \cdot b_{i}=q_{j} \cdot\left(r\left(q_{i}\right) q_{i}+r\left(q_{i \oplus}\right) q_{i \oplus}\right),
$$

which are easily verified. 
Lemma 2.3. Let $a_{k}, a_{m} \in \mathscr{A}$. Then

$$
\operatorname{ACD}\left(a_{k}\right) \cdot \operatorname{ACF}\left(a_{m}\right)-\operatorname{PCF}^{\prime}\left(a_{k}\right) \cdot \operatorname{PCF}\left(a_{m}\right)=-\delta_{k, m} .
$$

Proof. As for Lemma 2.2, the lemma is obvious if $c\left(a_{m}\right) \neq c\left(a_{k}\right)$, so assume that $c$ is a cycle with $c\left(a_{m}\right)=c=c\left(a_{k}\right)$.

If no edge-pair $q$ with $c(q)=c$ has a representative fixed by a nontrivial element of $G$, let $t, b_{1}, \ldots, b_{t}, q_{1}, \ldots, q_{t}$ be defined as in the proof of Lemma 2.2. Let $a_{k}=b_{i}$ and let $a_{m}=b_{j}$. In this case the proof reduces to showing that (in the notation of the proof of Lemma 2.2) $b_{i} \cdot\left(b_{j-}+b_{j}+b_{j+}\right)-\left(q_{i}+\right.$ $\left.q_{i+}\right) \cdot\left(q_{j}+q_{j+}\right)=-\delta_{i, j}$, which is clear since $\delta_{i, j}=\delta_{i+, j+}$.

If there is an edge-pair $q$ with $c(q)=c$ which has a representative fixed by a nontrivial element of $G$, let $t, b_{1}, \ldots, b_{t}, q_{1}, \ldots, q_{t+1}$ be defined as in the proof of Lemma 2.2. Let $a_{k}=b_{i}$ and let $a_{m}=b_{j}$. In this case the proof reduces to showing that (in the notation of the proof of Lemma 2.2)

$$
b_{i} \cdot\left(b_{j-}+b_{j}+b_{j+}\right)-\left(r\left(q_{i}\right) q_{i}+r\left(q_{i \oplus}\right) q_{i \oplus}\right) \cdot\left(q_{j}+q_{j \oplus}\right)=-\delta_{i, j} .
$$

By symmetry, we can assume that $1 \leq i \leq(t+1) / 2$. The equation is clear if $t=1$, so assume that $t \geq 2$. If $i=1$, the equation is

$$
b_{1} \cdot\left(b_{j-}+b_{j}+b_{j+}\right)-\left(2 q_{1}+q_{2}\right) \cdot\left(q_{j}+q_{j \oplus}\right)=-\delta_{1, j},
$$

which is easily checked by considering the cases $j=1, j=2$, and $j \geq 3$. If $i \geq 2$, then $t \geq 3$ and the equation is

$$
b_{i} \cdot\left(b_{j-}+b_{j}+b_{j+}\right)-\left(q_{i}+q_{i+1}\right) \cdot\left(q_{j}+q_{j \oplus}\right)=-\delta_{i, j} .
$$

This follows by examining the possibilites for $j$.

Lemma 2.4. Let $p_{k}, p_{m} \in \mathscr{P}$. Then

$$
\operatorname{ACF}\left(p_{k}\right) \cdot \operatorname{ACF}\left(p_{m}\right)-\operatorname{PCF}^{\prime}\left(p_{k}\right) \operatorname{PCD}\left(p_{m}\right)=r\left(p_{k}\right) \delta_{k, m} .
$$

Proof. As for Lemma 2.2, the lemma is obvious if $c\left(p_{k}\right) \neq c\left(p_{m}\right)$, so assume that $c$ is a cycle with $c\left(p_{k}\right)=c=c\left(p_{m}\right)$.

If no edge-pair $q$ with $c(q)=c$ has a representative fixed by a nontrivial element of $G$, let $t, b_{1}, \ldots, b_{t}, q_{1}, \ldots, q_{t}$ be defined as in the proof of Lemma 2.2. Let $p_{k}=q_{i}$ and let $p_{m}=q_{j}$. Since (1) holds, in this case the proof reduces to showing that (in the notation of Lemma 2.2) $\left(b_{i-}+b_{i}\right) \cdot\left(b_{j-}+b_{j}\right)-\left(q_{i-}+\right.$ $\left.q_{i}+q_{i+}\right) \cdot q_{j}=\delta_{i, j}$, which is clear since $\delta_{i, j}=\delta_{i-, j-}$.

If there is an edge-pair $q$ with $c(q)=c$ which has a representative fixed by a nontrivial element of $G$, let $t, b_{1}, \ldots, b_{t}, q_{1}, \ldots, q_{t+1}$ be defined as in the proof of Lemma 2.2. Let $p_{k}=q_{i}$ and let $p_{m}=q_{j}$. By symmetry, we can assume that $1 \leq i \leq(t+2) / 2$. As for the previous cases, we can simplify the equation since (1) holds. If $t=1$, then the equation reduces to $2 b_{1} \cdot 2 b_{1}-$ $\left(2 q_{i}+4 q_{2-i}\right) \cdot q_{j}=2 \delta_{i, j}$, which is clearly true. If $t \geq 2$ and $j=t+1$, the equation reduces to (in the notation of Lemma 2.2)

$$
\left(b_{i-}+b_{i}\right) \cdot 2 b_{t}-\left(r\left(q_{i \ominus}\right) q_{i \ominus}+r\left(q_{i}\right) q_{i}+r\left(q_{i \oplus}\right) q_{i \oplus}\right) \cdot q_{t+1}=0,
$$

which is established by checking the two cases $i<t$ and $i=t$. Thus we can assume that $t \geq 2$ and $i, j \leq t$. Then the proof reduces to showing that

$$
\left(b_{i-}+b_{i}\right) \cdot\left(b_{j-}+b_{j}\right)-\left(r\left(q_{i \ominus}\right) q_{i \ominus}+r\left(q_{i}\right) q_{i}+r\left(q_{i \oplus}\right) q_{i \oplus}\right) \cdot q_{j}=r\left(q_{i}\right) \delta_{i, j} \text {. }
$$

Again, this is checked by examining the possible cases. 
We now show that $B=A^{-1}$. If $a_{j}, a_{k} \in \mathscr{A}$, then the entry of $A B$ in the $a_{j}$ th row and $a_{k}$ th column is

$$
\begin{aligned}
& \left(\sum_{a_{i} \in \mathscr{A}}\left(l\left(a_{i}\right)-2\right) a_{i}\right) \cdot \operatorname{ACF}\left(a_{k}\right)-\left(\sum_{p_{i} \in \mathscr{P}}\left(l\left(p_{i}\right)-3\right) p_{i}\right) \cdot \operatorname{PCF}\left(a_{k}\right) \\
& \quad-\operatorname{ACD}\left(a_{j}\right) \cdot \operatorname{ACF}\left(a_{k}\right)+\operatorname{PCF}^{\prime}\left(a_{j}\right) \cdot \operatorname{PCF}\left(a_{k}\right) \\
& \quad-\operatorname{ACF}\left(d\left(p 1\left(a_{j}\right)\right)\right) \cdot \operatorname{ACF}\left(a_{k}\right)+\operatorname{PCF}^{\prime}\left(d\left(p 1\left(a_{j}\right)\right)\right) \cdot \operatorname{PCF}\left(a_{k}\right) \\
& \quad-\operatorname{ACF}\left(d\left(p 2\left(a_{j}\right)\right)\right) \cdot \operatorname{ACF}\left(a_{k}\right)+\operatorname{PCF}^{\prime}\left(d\left(p 2\left(a_{j}\right)\right)\right) \cdot \operatorname{PCF}\left(a_{k}\right) .
\end{aligned}
$$

By Lemmas 2.2 and 2.3, this is $\left.\left(l\left(a_{k}\right)-2\right)\right)\left(l\left(a_{k}\right)-3\right)-\left(l\left(a_{k}\right)-3\right)\left(l\left(a_{k}\right)-2\right)+$ $\delta_{j, k}=\delta_{j, k}$. If $p_{j} \in \mathscr{P}$ and $a_{k} \in \mathscr{A}$, then the entry of $A B$ in the $p_{j}$ th row and $a_{k}$ th column is

$$
\frac{\operatorname{ACF}\left(d\left(p_{j}\right)\right)}{r\left(d\left(p_{j}\right)\right)} \cdot \operatorname{ACF}\left(a_{k}\right)-\frac{\operatorname{PCF}^{\prime}\left(d\left(p_{j}\right)\right)}{r\left(d\left(p_{j}\right)\right)} \cdot \operatorname{PCF}\left(a_{k}\right)
$$

This is 0 by Lemma 2.2. If $p_{j}, p_{k} \in \mathscr{P}$, then entry of $A B$ in the $p_{j}$ th row and $p_{k}$ th column is

$$
\begin{aligned}
& \frac{\operatorname{ACF}\left(d\left(p_{j}\right)\right)}{r\left(d\left(p_{j}\right)\right)} \cdot\left(\sum_{a_{i} \in \mathscr{A}}\left(3-l\left(a_{i}\right)\right) a_{i}\right)+\frac{\operatorname{PCF}^{\prime}\left(d\left(p_{j}\right)\right)}{r\left(d\left(p_{j}\right)\right)} \cdot\left(\sum_{p_{i} \in \mathscr{P}} \frac{l\left(p_{i}\right)-2}{r\left(p_{i}\right)} p_{i}\right) \\
& \quad+\frac{\operatorname{ACF}\left(d\left(p_{j}\right)\right)}{r\left(d\left(p_{j}\right)\right)} \cdot \operatorname{ACF}\left(d\left(p_{k}\right)\right)-\frac{\operatorname{PCF}^{\prime}\left(d\left(p_{j}\right)\right)}{r\left(d\left(p_{j}\right)\right)} \cdot \operatorname{PCD}\left(d\left(p_{k}\right)\right) \\
& \quad+\frac{\operatorname{ACF}\left(d\left(p_{j}\right)\right)}{r\left(d\left(p_{j}\right)\right)} \cdot \operatorname{ACF}\left(a 1\left(p_{j}\right)\right)-\frac{\operatorname{PCF}^{\prime}\left(d\left(p_{j}\right)\right)}{r\left(d\left(p_{j}\right)\right)} \cdot \operatorname{PCF}\left(a 1\left(p_{k}\right)\right) \\
&+\frac{\operatorname{ACF}\left(d\left(p_{j}\right)\right)}{r\left(d\left(p_{j}\right)\right)} \cdot \operatorname{ACF}\left(a 2\left(p_{j}\right)\right)-\frac{\operatorname{PCF}^{\prime}\left(d\left(p_{j}\right)\right)}{r\left(d\left(p_{j}\right)\right)} \cdot \operatorname{PCF}\left(a 2\left(p_{k}\right)\right) .
\end{aligned}
$$

The first line of the sum is 0 , and it follows from Lemmas 2.2 and 2.4 that the sum is $\delta_{j, k}$. If $a_{j} \in \mathscr{A}$ and $p_{k} \in \mathscr{P}$, the entry of $A B$ in the $a_{j}$ th row and $p_{k}$ th column is

$$
\begin{aligned}
& \left(\sum_{a_{i} \in \mathscr{A}}\left(l\left(a_{i}\right)-2\right) a_{i}\right)\left(\sum_{a_{i} \in \mathscr{A}}\left(3-l\left(a_{i}\right)\right) a_{i}\right) \\
& +\left(\sum_{p_{i} \in \mathscr{P}}\left(l\left(p_{i}\right)-3\right) p_{i}\right) \cdot\left(\sum_{p_{i} \in \mathscr{P}} \frac{l\left(p_{i}\right)-2}{r\left(p_{i}\right)} p_{i}\right) \\
& +\left(\sum_{a_{i} \in \mathscr{A}}\left(l\left(a_{i}\right)-2\right) a_{i}\right) \cdot \operatorname{ACF}\left(a 1\left(p_{k}\right)\right)-\left(\sum_{p_{i} \in \mathscr{P}}\left(l\left(p_{i}\right)-3\right) p_{i}\right) \cdot \operatorname{PCF}\left(a 1\left(p_{k}\right)\right)
\end{aligned}
$$




$$
\begin{aligned}
& +\left(\sum_{a_{i} \in \mathscr{A}}\left(l\left(a_{i}\right)-2\right) a_{i}\right) \cdot \operatorname{ACF}\left(a 2\left(p_{k}\right)\right)-\left(\sum_{p_{i} \in \mathscr{P}}\left(l\left(p_{i}\right)-3\right) p_{i}\right) \cdot \operatorname{PCF}\left(a 2\left(p_{k}\right)\right) \\
& -\left(\sum_{a_{i} \in \mathscr{A}}\left(3-l\left(a_{i}\right)\right) a_{i}\right) \cdot \operatorname{ACF}\left(d\left(p 1\left(a_{j}\right)\right)\right) \\
& -\left(\sum_{p_{i} \in \mathscr{P}} \frac{l\left(p_{i}\right)-2}{r\left(p_{i}\right)} p_{i}\right) \cdot \operatorname{PCF}^{\prime}\left(d\left(p 1\left(a_{j}\right)\right)\right) \\
& -\left(\sum_{a_{i} \in \mathscr{A}}\left(3-l\left(a_{i}\right)\right) a_{i}\right) \cdot \operatorname{ACF}\left(d\left(p 2\left(a_{j}\right)\right)\right) \\
& -\left(\sum_{p_{i} \in \mathscr{P}} \frac{l\left(p_{i}\right)-2}{r\left(p_{i}\right)} p_{i}\right) \cdot \operatorname{PCF}\left(d\left(p 2\left(a_{j}\right)\right)\right) \\
& +\left(\sum_{a_{i} \in \mathscr{A}}\left(l\left(a_{i}\right)-2\right) a_{i}\right) \cdot \operatorname{ACF}\left(d\left(p_{k}\right)\right)-\left(\sum_{p_{i} \in \mathscr{P}}\left(l\left(p_{i}\right)-3\right) p_{i}\right) \cdot \operatorname{PCD}\left(d\left(p_{k}\right)\right) \\
& -\left(\sum_{a_{i} \in \mathscr{A}}\left(3-l\left(a_{i}\right)\right) a_{i}\right) \cdot \operatorname{ACD}\left(a_{j}\right)-\left(\sum_{p_{i} \in \mathscr{P}} \frac{l\left(p_{i}\right)-2}{r\left(p_{i}\right)} p_{i}\right) \cdot \operatorname{PCF}\left(a_{j}\right) \\
& -\operatorname{ACD}\left(a_{j}\right) \cdot \operatorname{ACF}\left(d\left(p_{k}\right)\right)+\operatorname{PCF}\left(a_{j}\right) \cdot \operatorname{PCD}\left(d\left(p_{k}\right)\right. \\
& -\operatorname{ACF}\left(d\left(p 1\left(a_{j}\right)\right)\right) \cdot \operatorname{ACF}\left(a 1\left(p_{k}\right)\right)+\operatorname{PCF}^{\prime}\left(d\left(p 1\left(a_{j}\right)\right)\right) \cdot \operatorname{PCF}\left(a 1\left(p_{k}\right)\right) \\
& -\operatorname{ACF}\left(d\left(p 1\left(a_{j}\right)\right)\right) \cdot \operatorname{ACF}\left(a 2\left(p_{k}\right)\right)+\operatorname{PCF}^{\prime}\left(d\left(p 1\left(a_{j}\right)\right)\right) \cdot \operatorname{PCF}\left(a 2\left(p_{k}\right)\right) \\
& -\operatorname{ACF}\left(d\left(p 2\left(a_{j}\right)\right)\right) \cdot \operatorname{ACF}\left(a 1\left(p_{k}\right)\right)+\operatorname{PCF}^{\prime}\left(d\left(p 2\left(a_{j}\right)\right)\right) \cdot \operatorname{PCF}\left(a 1\left(p_{k}\right)\right) \\
& -\operatorname{ACF}\left(d\left(p 2\left(a_{j}\right)\right)\right) \cdot \operatorname{ACF}\left(a 2\left(p_{k}\right)\right)+\operatorname{PCF}^{\prime}\left(d\left(p 2\left(a_{j}\right)\right)\right) \cdot \operatorname{PCF}\left(a 2\left(p_{k}\right)\right) \\
& -\operatorname{ACD}\left(a_{j}\right) \cdot \operatorname{ACF}\left(a 1\left(p_{k}\right)\right)+\operatorname{PCF}\left(a_{j}\right) \cdot \operatorname{PCF}\left(a 1\left(p_{k}\right)\right) \\
& -\operatorname{ACD}\left(a_{j}\right) \cdot \operatorname{ACF}\left(a 2\left(p_{k}\right)\right)+\operatorname{PCF}^{\prime}\left(a_{j}\right) \cdot \operatorname{PCF}\left(a 2\left(p_{k}\right)\right) \\
& -\operatorname{ACF}\left(d\left(p 1\left(a_{j}\right)\right)\right) \cdot \operatorname{ACF}\left(d\left(p_{k}\right)\right)+\operatorname{PCF}^{\prime}\left(d\left(p 1\left(a_{j}\right)\right)\right) \cdot \operatorname{PCD}\left(d\left(p_{k}\right)\right) \\
& -\operatorname{ACF}\left(d\left(p 2\left(a_{j}\right)\right)\right) \cdot \operatorname{ACF}\left(d\left(p_{k}\right)\right)+\operatorname{PCF}^{\prime}\left(d\left(p 2\left(a_{j}\right)\right)\right) \cdot \operatorname{PCD}\left(d\left(p_{k}\right)\right) \\
&
\end{aligned}
$$

The first 5 lines are 0 , line 6 is 1 , line 7 is -1 , and lines $8-12$ are 0 by Lemma 2.2. By Lemmas 2.3 and 2.4, the sum of the last 4 lines is 0 . Thus $A B=I$ and $B=A^{-1}$.

\section{THE PROOF OF THE RECIPROCITY THEOREM}

Let $G$ be a cocompact group of isometries of $\mathbb{E}^{2}$ or $\mathbb{H}^{2}$ and let $D$ be a Dirichlet region for the action of $G$. We assume, as we did for $\S 2$, that either $D$ has at least six sides, $D$ has four sides and no vertex of $D$ has valence 3, or $D$ has five sides and $D$ does not have three vertices that are not adjacent and that each have valence three. In this section we prove that the growth function $f$ is reciprocal. The proof follows immediately from Lemma 3.5 and the following lemma from [5]. 
Lemma 3.1. Let $f(z)=1+U(I-z A)^{-1} z V$, where $A$ is an invertible square matrix, $U$ is a row vector, and $V$ is a column vector, and let $W=A^{-1} V$. Then $f(z)=f\left(z^{-1}\right)$ for all $z \neq 0$ if and only if $U A^{n} W=-U A^{-n} W$ for all nonnegative integers $n$.

Proof. The Maclaurin series for $f(z)$ is

$$
1+\sum_{n=0}^{\infty} U A^{n} V z^{n+1}=1+\sum_{n=1}^{\infty} U A^{n} W z^{n} .
$$

Since

$$
\begin{aligned}
f\left(z^{-1}\right) & =1+U\left(I-z^{-1} A\right)^{-1} z^{-1} V=1+U\left(A^{-1}-z^{-1} I\right)^{-1} A^{-1} V z^{-1} \\
& =1-U\left(I-z A^{-1}\right)^{-1} W,
\end{aligned}
$$

the Maclaurin series for $f\left(z^{-1}\right)$ is $1-U W-\sum_{n=1}^{\infty} U A^{-n} W z^{n}$. The result follows by comparing the two series term by term.

For each nonnegative integer $n, a \in \mathscr{A}$, and $p \in \mathscr{P}$, let $\alpha(n, a), \gamma(n, a)$, $\beta(n, p)$, and $\delta(n, p)$ be the integers defined by

$$
A^{n} W=\sum_{i=1}^{r} \alpha\left(n, a_{i}\right) a_{i}+\sum_{i=1}^{s} \beta\left(n, p_{i}\right) p_{i}
$$

and

$$
A^{-n} W=\sum_{i=1}^{r} \gamma\left(n, a_{i}\right) a_{i}+\sum_{i=1}^{s} \delta\left(n, p_{i}\right) p_{i} .
$$

Given a nonnegative integer $n$ and $a \in \mathscr{A}$, let

$$
\kappa(n, a)=\alpha(n, a)+r(p 1(a)) \beta(n, p 1(a))+r(p 2(a)) \beta(n, p 2(a))
$$

and let

$$
\lambda(n, a)=\gamma(n, a)+r(p 1(a)) \delta(n, p 1(a))+r(p 2(a)) \delta(n, p 2(a)) .
$$

Lemma 3.2. If $n$ is a nonnegative integer, then $\kappa(n, a)$ depends only on the cycle containing $a$.

Proof. The proof is by induction on $n$. If $n=0$ and $a \in \mathscr{A}$, then $\kappa(n, a)=1$ and the result is obvious. Assume that $n \geq 0$ and that for each $a \in \mathscr{A}, \kappa(n, a)$ depends only on the cycle of $a$. Let $a \in \mathscr{A}$. Then

$$
\begin{aligned}
\kappa(n+1, a)= & \sum_{i=1}^{r} \alpha\left(n, a_{i}\right)\left(l\left(a_{i}\right)-2\right)+\sum_{i=1}^{s} \beta\left(n, p_{i}\right)\left(l\left(p_{i}\right)-3\right) \\
& -\sum_{b \in a c(a)} \alpha(n,[b])-\sum_{p \in p c(a)} r([p]) \beta(n,[p])+\kappa(n, a)
\end{aligned}
$$

depends only on the cycle of $a$.

Lemma 3.3. If $n$ is a nonnegative integer and $a \in \mathscr{A}$, then $\lambda(n, a)$ depends only on the cycle containing $a$.

Proof. The proof is by induction on $n$. If $n=0$ and $a \in \mathscr{A}$, then $\lambda(n, a)=1$ and the result is obvious. Assume that $n \geq 0$ and that if $a \in \mathscr{A}$ then $\lambda(n, a)$ 
depends only on the cycle of $a$. Let $a \in \mathscr{A}$. Then $\lambda(n+1, a)=X \cdot A^{-n} U$, where

$$
\begin{aligned}
X= & \operatorname{ACF}(a)-\operatorname{ACF}(p 1(a))-\operatorname{ACF}(p 2(a)) \\
& +\sum_{i=1}^{s}(-l(a)+l(p 1(a))+l(p 2(a))-1) p_{i} \\
& +\operatorname{PCF}^{\prime}(p 1(a))-\operatorname{PCF}^{\prime}(a 1(p 1(a)))-\operatorname{PCF}^{\prime}(a 2(p 1(a))) \\
& +\operatorname{PCF}^{\prime}(p 2(a))-\operatorname{PCF}^{\prime}(a 1(p 2(a)))-\operatorname{PCF}^{\prime}(a 2(p 2(a))) \\
& +\sum_{p \in \operatorname{pcf}(a)} r([p]) d([p])-\sum_{p \in \operatorname{pcd}(p 1(a))} r([p]) d([p])-\sum_{p \in \operatorname{pcd}(p 2(a))} r([p]) d([p]) \\
= & \sum_{i=1}^{s}(l(a)-1) p_{i}-A C(a)-2 P C^{\prime}(a) \\
& -\sum_{p \in p c(a)} r([p]) d([p])+a+r(p 1(a)) p 1(a)+r(p 2(a) p 2(a),
\end{aligned}
$$

so

$$
\begin{aligned}
\lambda(n+1, a)= & (l(a)-1) \sum_{i=1}^{s} \delta\left(n, p_{i}\right)-\sum_{b \in a c(a)} \gamma(n,[b]) \\
& -2 \sum_{p \in p c(a)} r([p]) \delta(n,[p])-\sum_{p \in p c(a)} r([p]) \delta(n, d([p]))+\lambda(n, a) \\
= & (l(a)-1) \sum_{i=1}^{s} \delta\left(n, p_{i}\right)-(l(a)-1) \lambda(n, a) \\
& -\sum_{p \in p c(a)} r([p]) \delta(n, d([p])),
\end{aligned}
$$

which depends only on the cycle of $a$.

Lemma 3.4. For all $a \in \mathscr{A}, p \in \mathscr{P}$, and nonnegative integers $n, \beta(n, d(p))=$ $-\delta(n, p)$ and $\kappa(n, a)=\lambda(n, a)-\sum_{i=1}^{s} \delta(n, p)$.

Proof. The proof is by induction on $n$. The case $n=0$ is obvious since $\alpha(0, a)=\gamma(0, a)=1$ and $\beta(0, p)=\delta(0, p)=0$. Assume that $n$ is a nonnegative integer such that $\beta(n, d(p))=-\delta(n, p)$ and $\kappa(n, a)=\lambda(n, a)-$ $\sum_{i=1}^{s} \delta(n, p)$ for all $a \in \mathscr{A}$ and $p \in \mathscr{P}$. Let $a \in \mathscr{A}$ and let $p \in \mathscr{P}$. Let $\alpha=\sum_{i=1}^{r} \alpha\left(n, a_{i}\right) a_{i}$, let $\beta=\sum_{i=1}^{s} \beta\left(n, p_{i}\right) p_{i}$, let $\gamma=\sum_{i=1}^{r} \gamma\left(n, a_{i}\right) a_{i}$, and let $\delta=\sum_{i=1}^{s} \delta\left(n, p_{i}\right) p_{i}$. Then

$$
\begin{aligned}
r(p) & (\beta(n+1, d(p))+\delta(n+1, p)) \\
= & \operatorname{ACF}(p) \cdot \alpha+\operatorname{PCF}^{\prime}(p) \cdot \beta-\sum_{q \in p c d(p)} r([q]) \delta(n, d([q]))-\operatorname{ACF}(p) \cdot \gamma \\
& -\operatorname{PCF}^{\prime}(a 1(p)) \cdot \delta-\operatorname{PCF}^{\prime}(a 2(p)) \cdot \delta+\sum_{i=1}^{s}(l(p)-2) \delta\left(n, p_{i}\right) \\
= & (l(p)-2) \kappa(n, b)-(l(p)-2) \gamma(n, b)+(l(p)-2) \sum_{i=1}^{s} \delta\left(n, p_{i}\right)=0
\end{aligned}
$$


for some $b \in \mathscr{A}$ with $c(b)=c(p)$ by the induction hypotheses, so $\beta(n+1, d(p))=-\delta(n+1, p)$.

$$
\begin{aligned}
\kappa(n+ & 1, a)-\gamma(n+1, a)+\sum_{i=1}^{s} \delta\left(n+1, p_{i}\right) \\
= & \kappa(n+1, a)-\gamma(n+1, a)-\sum_{i=1}^{s} \beta\left(n+1, d\left(p_{i}\right)\right) \\
= & \sum_{i=1}^{r}\left(l\left(a_{i}\right)-2\right) \alpha\left(n, a_{i}\right)+\sum_{i=1}^{s}\left(l\left(p_{i}\right)-3\right) \beta\left(n, p_{i}\right) \\
& -\sum_{b \in a c(a)} \alpha(n,[b])-\sum_{q \in p c(a)} r([q]) \beta(n,[q])+\kappa(n, a) \\
& -(l(a)-1) \sum_{i=1}^{s} \delta\left(n, p_{i}\right)+(l(a)-1) \lambda(n, a) \\
& -\sum_{q \in p c(a)} r([q]) \beta(n,[q])-\sum_{i=1}^{s} \frac{\mathrm{ACF}\left(p_{i}\right)}{r\left(p_{i}\right)} \cdot \alpha-\sum_{i=1}^{s} \frac{\operatorname{PCF}^{\prime}\left(p_{i}\right)}{r\left(p_{i}\right)} \cdot \beta \\
= & \sum_{i=1}^{r}\left(l\left(a_{i}\right)-2\right) \alpha\left(n, a_{i}\right)-\sum_{i=1}^{s} \frac{\operatorname{ACF}\left(p_{i}\right)}{r\left(p_{i}\right)} \cdot \alpha \\
& +\sum_{i=1}^{s}\left(l\left(p_{i}\right)-3\right) \beta\left(n, p_{i}\right)-\sum_{i=1}^{s} \frac{\operatorname{PCF}^{\prime}\left(p_{i}\right)}{r\left(p_{i}\right)} \cdot \beta \\
& -(l(a)-1) \kappa(n, a)-(l(a)-1) \sum_{i=1}^{s} \delta\left(n, p_{i}\right)+(l(a)-1) \lambda(n, a) \\
= & 0 . \quad \square
\end{aligned}
$$

Lemma 3.5. For all nonnegative integers $n, U A^{n} W=-U A^{-n} W$.

Proof. If $n$ is a nonnegative integer, then

$$
U A^{n} W=\sum_{p \in \mathscr{P}} \beta(n, p)=\sum_{p \in \mathscr{P}}-\delta(n, d(p))=\sum_{p \in \mathscr{P}}-\delta(n, p)=-U A^{-n} W,
$$

where the second equality follows from Lemma 3.4.

\section{TRIANGULAR DOMAINS}

If the fundamental domain $D$ is a triangle, it will no longer be true that the vertices of $\partial B(n)$ will have valence one or two in $B(n)$. A vertex $v$ in $\partial B(n)$ will have valence one only if $n=0$. As seen in Figure 5, if $e$ is an edge in $\partial B(n)$ and $g \in G$ with $|g|=n+1$ and $g D \cap B(n)=e$, then the vertex $v$ of $g D$ with $v \notin e$ will have valence at least three in $B(n+1)$. With the full geometric generating set, $g D$ will be a buried domain exactly if $v \in \operatorname{int}(B(n+1))$. Figure 5 illustrates how this occurs if $D$ has an angle of measure $\pi / 2$ or $2 \pi / 3$. Our analysis will show that there are buried domains 

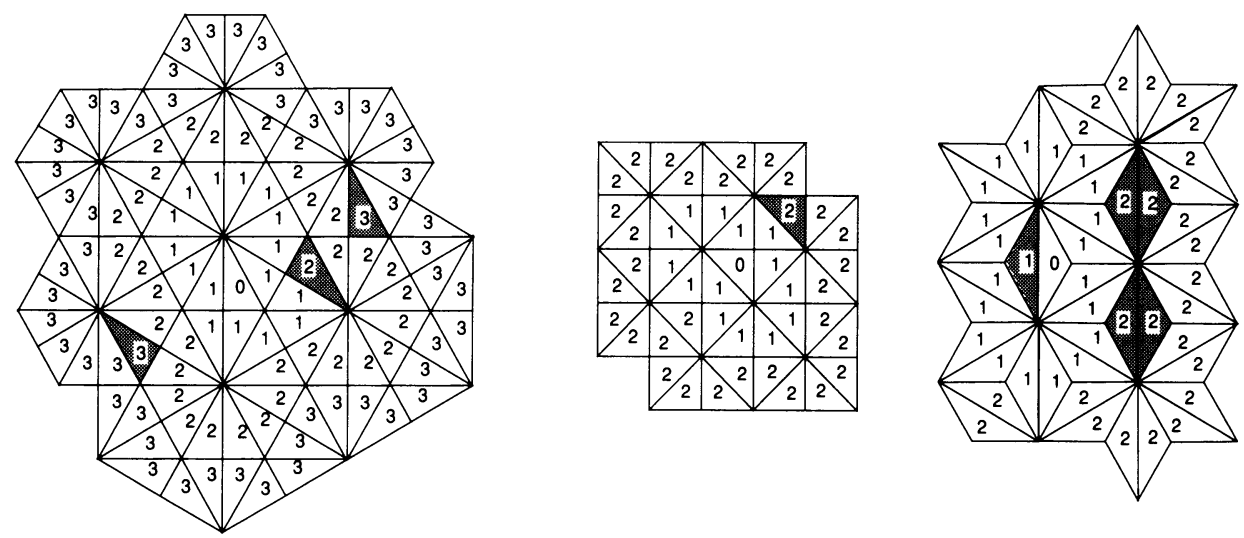

Figure 5. $\Delta^{*}(2,3,6), \Delta^{*}(2,4,4)$, AND $\Delta(2,3,6)$

exactly if one of the vertices of $D$ has angle measure $\pi / 2$ or $2 \pi / 3$, and that reciprocity of the growth function fails in exactly these cases.

Let $G$ be a cocompact, discrete group of isometries of $\mathbb{E}^{2}$ or $\mathbb{H}^{2}$ with fundamental domain $D$ a triangle. We can assume without loss of generality that the measure of an angle of the triangle is $2 \pi / p$, where $p$ is the valence of the vertex in the tessellation $\{g D: g \in G\}$. Let $v_{1}, v_{2}$, and $v_{3}$ be the vertices of $D$, and let $e_{1}, e_{2}$, and $e_{3}$ be the edges of $D$, chosen so that $\partial e_{1}=v_{1} \cup v_{2}$, $\partial e_{2}=v_{2} \cup v_{3}$, and $\partial e_{3}=v_{3} \cup v_{1}$. The combinatorics of the combinatorial balls is not as simple as in the earlier sections, but the analysis is still tractable since there are so few sides. We will do this by cases as follows:

(i) angle measures $\pi / p, \pi / q$, and $\pi / r$, with $p, q, r \geq 3$;

(ii) all angle measures $2 \pi / 3 p$, with $p \geq 3, p$ odd;

(iii) angle measures $2 \pi /(2 q+1), \pi / p$, and $\pi / p$, with $q \geq 3$ and $p \geq 3$;

(iv) angle measures $2 \pi / 5, \pi / p$, and $\pi / p$, with $p \geq 4$;

(v) angle measures $\pi / 2, \pi / p$, and $\pi / q$, with $p, q \geq 4$;

(vi) angle measures $\pi / 2, \pi / 3$, and $\pi / q$, with $q \geq 6$; and

(vii) angle measures $2 \pi / 3, \pi / p$, and $\pi / p$, with $p \geq 6$.

We start with an analogue of Lemma 1.2.

Lemma 4.1. Let $X$ be $\mathbb{E}^{2}$ or $\mathbb{H}^{2}$, let $G$ be a cocompact group of isometries of $X$, and let $D$ be a Dirichlet region for the action of $G$ such that $D$ is a triangle and each vertex of $D$ has angle measure a submultiple of $\pi$. Let $B$ be a convex subset of $X$ which is a union of images of $D$ under the action of $G$, and let $B^{\prime}=B \cup\{g D: g \in G$ and $g D \cap B \neq \varnothing\}$. Let $g, h \in G$ with $g D \subset B^{\prime}-\operatorname{int}(B)$ and $h D \subset B^{\prime}-\operatorname{int}(B)$. Then

(i) $g D \cap B$ is connected,

(ii) if $g D \cap h D \neq \varnothing$, then either

(a) $g D \cap h D$ contains a vertex of $B$,

(b) there is an element $g^{\prime} \in G$ such that the vertices of $g^{\prime} D$ are $g D \cap$ $h D, g D \cap B$, and $h D \cap B$, or

(c) there are elements $g^{\prime}, h^{\prime} \in G$ such that $g^{\prime} D \cap h^{\prime} D$ contains a vertex $v$ of $\partial B$ with valence 2 in $B$ and angle measure $\pi$ in $B$ and the vertices of $g^{\prime} D \cup h^{\prime} D$ are $v, g D \cap h D, g D \cap B$, and $h D \cap B$, 
(iii) $B^{\prime}$ is homeomorphic to a ball, and

(iv) each vertex of $\partial B^{\prime}$ has valence 2,3 , or 4 in $B^{\prime}$.

Proof. Since $g D$ and $B$ are convex, $g D \cap B$ is convex and hence is connected. Suppose that $g D \cap h D \neq \varnothing$ and $g D \cap h D$ does not contain a vertex of $B$. Then there is a polygon $C$ such that $\operatorname{int}(C)$ is a component of $X-(g D \cup h D \cup B)$. Since $B$ is convex and the angles of $D$ have measures at most $\pi / 2, g D \cap B$ and $h D \cap B$ are vertices. Let $e$ be the edge of $C \cap B$ which contains the vertex $g D \cap B$, and let $e^{\prime}$ be the edge of $C \cap B$ which contains the vertex $h D \cap B$. Let $g^{\prime}$ be the element of $G$ such that $g^{\prime} D \subset C$ and $e \subset g^{\prime} D$, and let $h^{\prime}$ be the element of $G$ such that $h^{\prime} D \subset C$ and $e^{\prime} \subset h^{\prime} D$. Let $w$ be the vertex of $g^{\prime} D$ that is not in $B$ and let $w^{\prime}$ be the vertex of $h^{\prime} D$ that is not in $B$. Since each vertex of $D$ has angle measure a submultiple of $\pi$, each edge of $g^{\prime} D$ and $h^{\prime} D$ is contained in a geodesic which lies in the 1-skeleton of the tessellation. Thus $w=w^{\prime}$. If $g^{\prime}=h^{\prime}$ then we have case (ii.b), and if $g^{\prime} \neq h^{\prime}$ we have case (ii.c). Property (iii) now follows as in the proof of Lemma 1.1, and (iv) follows from (ii).

We first do case (i). Suppose that the angle measures at $v_{1}, v_{2}$, and $v_{3}$ are $\pi / p, \pi / q$, and $\pi / r$ (respectively), with $p, q, r \geq 3$. Using Lemma 4.1, it follows by induction that for every positive integer $n, B(n)$ is convex and each vertex of $B(n)$ has valence 2 or 3 in $B(n)$. We will compute the growth series and hence the growth function by putting a linear recursion on the vertices of the boundaries of the combinatorial balls. Since when $D$ is a triangle the tessellation depends only on $D$ and not on the group $G$, we may assume that $G$ is a Coxeter group and that the vertices of $D$ are in distinct orbits under the action of $G$ (this is convenient for describing the types). We will divide the boundary vertices into nine types. Let $n$ be a positive integer, and let $w$ be a vertex of $\partial B(n)$. The vertex $w$ is of type $\tau_{1}\left(\tau_{2}\right)$ if $w \sim_{G} v_{1}, w$ has valence 2 in $B(n)$, and the two vertices adjacent to $w$ in $\partial B(n)$ are $G$-equivalent to $v_{2} \quad\left(v_{3}\right)$. The vertex $w$ is of type $\tau_{3}$ if $w \sim_{G} v_{1}$ and $w$ has valence 3 in $B(n)$. The vertex $w$ is of type $\tau_{4}\left(\tau_{5}\right)$ if $w \sim_{G} v_{2}, w$ has valence 2 in $B(n)$, and the two vertices adjacent to $w$ in $\partial B(n)$ are $G$-equivalent to $v_{1}\left(v_{3}\right)$. The vertex $w$ is of type $\tau_{6}$ if $w \sim_{G} v_{2}$ and $w$ has valence 3 in $B(n)$. The vertex $w$ is of type $\tau_{7}\left(\tau_{8}\right)$ if $w \sim_{G} v_{3}, w$ has valence 2 in $B(n)$, and the two vertices adjacent to $w$ in $\partial B(n)$ are $G$-equivalent to $v_{1}\left(v_{2}\right)$. The vertex $w$ is of type $\tau_{9}$ if $w \sim_{G} v_{3}$ and $w$ has valence 3 in $B(n)$.

Given a vertex $w$ of $\partial B(n)$ of type $\tau_{j}$, let $b_{i j}$ be the number of vertices of $\partial B(n+1)$ that have type $\tau_{i}$ and are connected by an edge to $w$, and let $m(j)$ be the number of vertices of $\partial B(n-1)$ that are connected by an edge to $w$. It is straightforward to show that $b_{i j}$ and $m(j)$ depend only on the type of $w$ and not on $n$ or the particular element $w$. The recursion matrix $A$ has entry $a_{i j}=b_{i j} / m(i)$. The initial vector $V$ has entry $v_{j}$ the number of elements of type $\tau_{j}$ of length 1 . For example, if $w$ is a vertex of $\partial B(n)$ of type $\tau_{1}$, then there are $p$ edges in $B(n+1)$ which have one boundary vertex $w$ and are $G$-equivalent to $e_{1}$. Two of these edges are in $\partial B(n)$, and the other $p-2$ will have other boundary vertex a vertex in $\partial B(n+1)$ of type $\tau_{5}$. There are also $p$ edges in $B(n+1)$ that have one boundary vertex $w$ and are $G$-equivalent to $e_{2}$. One of these is in $\operatorname{int}(B(n+1))$, two of these have other vertex a vertex in $\partial B(n+1)$ of type $\tau_{9}$, and the other $p-3$ have other vertex a boundary 
vertex of $B(n+1)$ of type $\tau_{8}$. Since vertices with valence 3 are produced by two elements of the previous length, the first column of the recursion matrix $A$ is the column vector with nonzero entries $p-2$ in the fifth row, $p-3$ in the eighth row, and $1=2 \cdot \frac{1}{2}$ in the ninth row. By similar arguments one shows that the recursion matrix is

$$
A=\left[\begin{array}{ccccccccc}
0 & 0 & 0 & 0 & 0 & 0 & r-2 & r-3 & r-3 \\
0 & 0 & 0 & q-2 & q-3 & q-3 & 0 & 0 & 0 \\
0 & 0 & 0 & 0 & 1 & 1 / 2 & 0 & 1 & 1 / 2 \\
0 & 0 & 0 & 0 & 0 & 0 & r-3 & r-2 & r-3 \\
p-2 & p-3 & p-3 & 0 & 0 & 0 & 0 & 0 & 0 \\
0 & 1 & 1 / 2 & 0 & 0 & 0 & 1 & 0 & 1 / 2 \\
0 & 0 & 0 & q-3 & q-2 & q-3 & 0 & 0 & 0 \\
p-3 & p-2 & p-3 & 0 & 0 & 0 & 0 & 0 & 0 \\
1 & 0 & 1 / 2 & 1 & 0 & 1 / 2 & 0 & 0 & 0
\end{array}\right]
$$

and the transpose of the initial vector $V$ is

$$
\left[\begin{array}{lllllllll}
r-2 & q-2 & 1 & r-2 & p-2 & 1 & q-2 & p-2 & 1
\end{array}\right] .
$$

For every positive integer $n, A^{n-1} V$ gives the number of vertices of $\partial B(n)$ by type. If $n$ is a positive integer and $g \in G$ with $|g|=n$, then either $g D \cap \partial B(n)$ is a vertex and this vertex has valence 3 in $\partial B(n)$ or $g D \cap \partial B(n)$ is an edge. A vertex $w \in \partial B(n)$ of valence 2 in $B(n)$ is in the boundary of two domains $g_{1} D$ and $g_{2} D$ with $\left|g_{i}\right|=n$, and $g_{1} D$ and $g_{2} D$ each have two vertices in $\partial B(n)$. A vertex $w \in \partial B(n)$ of valence 3 in $B(n)$ is in the boundary of three domains $g D$ with $|g|=n$; two of these have two vertices in $\partial B(n)$ and the third has one vertex in $\partial B(n)$. Thus the number of elements of $G$ of length $n$ is $U A^{n-1} V$, where

$$
U=\left[\begin{array}{lllllllll}
1 & 1 & 2 & 1 & 1 & 2 & 1 & 1 & 2
\end{array}\right] .
$$

The growth function

$$
f(z)=\frac{\left(z^{6}+1\right)+\left(2 s_{1}-6\right)\left(z^{5}+z\right)+\left(3 s_{2}-8 s_{1}+15\right)\left(z^{4}+z^{2}\right)+\left(4 s_{3}-6 s_{2}+12 s_{1}-20\right) z^{3}}{z^{6}+\left(4 s_{1}-s_{2}-9\right) z^{4}+\left(4 s_{2}-2 s_{3}-8 s_{1}+16\right) z^{3}+\left(4 s_{1}-s_{2}-9\right) z^{2}+1},
$$

where $s_{1}=p+q+r, s_{2}=p q+p r+q r$, and $s_{3}=p q r$.

We next do case (ii), where the three vertices of $D$ are in the same $G$-orbit and each has angle measure $2 \pi / 3 p$, where $p$ is an odd integer and $p \geq 3$. Using Euler characteristic arguments as in $\S 1$, it is straightforward to show inductively that if $n$ is a positive integer then $B(n)$ is convex and each vertex of $\partial B(n)$ has valence 2 or 3 in $B(n)$. In this case our linear recursion has two types. A vertex $v$ of $\partial B(n)$ has type $\tau_{1}$ if it has valence two in $B(n)$ and has type $\tau_{2}$ if it has valence 3 . The recursion matrix $A$, initial vector $V$, and weight vector $U$ are

$$
A=\left[\begin{array}{cc}
3 p-5 & 3 p-6 \\
1 & 1
\end{array}\right], \quad V=\left[\begin{array}{c}
9 p-12 \\
3
\end{array}\right], \quad \text { and } \quad U=\left[\begin{array}{ll}
1 & 2
\end{array}\right] .
$$

The growth function

$$
f(z)=\frac{z^{2}+(6 p-2) z+1}{z^{2}+(4-3 p) z+1}
$$


The final case with each combinatorial ball convex is case (iii), where the vertex $v_{1}$ of $D$ has angle measure $2 \pi /(2 q+1), q \geq 3$, and the vertices $v_{2}$ and $v_{3}$ are in the same $G$-orbit and each have angle measure $\pi / p, p \geq 3$. Again, one can show inductively that if $n$ is a positive integer then $B(n)$ is convex and each vertex of $\partial B(n)$ has valence 2 or 3 in $B(n)$. The linear recursion has five types. A vertex $v$ of $\partial B(n)$ has type $\tau_{1}\left(\tau_{2}\right)$ if $v \sim_{G} v_{1}$ and $v$ has valence $2(3)$ in $B(n)$. The vertex $v$ is of type $\tau_{3}\left(\tau_{4}\right)$ if $v \sim_{G} v_{2}$ has valence 2 in $B(n)$, and the two vertices adjacent to it in $\partial B(n)$ each are $G$-equivalent to $v_{1}\left(v_{2}\right)$. The vertex $v$ is of type $\tau_{5}$ if $v \sim_{G} v_{2}$ and $v$ has valence 3 in $B(n)$. The weight vector $U=\left[\begin{array}{lllll}1 & 2 & 1 & 1 & 2\end{array}\right]$, and the recursion matrix $A$ and initial vector $V$ are

$$
A=\left[\begin{array}{ccccc}
0 & 0 & p-2 & p-3 & p-3 \\
0 & 0 & 0 & 1 & 1 / 2 \\
0 & 0 & p-3 & p-2 & p-3 \\
2 q-4 & 2 q-5 & 0 & 0 & 0 \\
1 & 1 & 1 & 0 & 1 / 2
\end{array}\right] \quad \text { and } \quad V=\left[\begin{array}{c}
2 p-4 \\
1 \\
2 p-4 \\
2 q-3 \\
2
\end{array}\right]
$$

The growth function

$$
f(z)=\frac{z^{4}+(2 q+3 p-3) z^{3}+(4 p q-4 p-4 q+4) z^{2}+(2 q+3 p-3) z+1}{z^{4}+(2-p) z^{3}+(-2 p q+4 q+3 p-4) z^{2}+(2-p) z+1}
$$

Case (iv) is similar to case (iii). In case (iv) $v_{1}$ has angle measure $2 \pi / 5$, $v_{2}$ and $v_{3}$ are $G$-equivalent, and $v_{2}$ and $v_{3}$ each have angle measure $\pi / p$ with $p \geq 4$. The types from case (iii) each have analogues here, but there are additional types. The combinatorial balls need not be convex because of vertices in $\partial B(n)$ with valence 3 in $B(n)$ and angle measure $6 \pi / 5$, and vertices with angle measure $6 \pi / 5$ produce vertices at the next stage with angle measure $4 \pi / p$. There is more collapsing than usual since the combinatorial balls are not convex and there are such few sides to $D$, and so the types need to include more information about adjacent vertices. Let $n$ be a positive integer such that $B(n)$ is homeomorphic to a ball, and let $v$ be a vertex of $\partial B(n)$. Then $v$ has type $\tau_{1}\left(\tau_{2}\right)$ if $v \sim_{G} v_{1}$ and $v$ has valence $2(3)$ in $B(n)$. If $v \sim_{G} v_{2}$, $v$ has valence 2 in $B(n)$, and the two vertices adjacent to it in $\partial B(n)$ are $G$-equivalent to $v_{1}$, then $v$ has type $\tau_{3}$ if the two adjacent vertices have type $\tau_{1}$ and $v$ has type $\tau_{4}$ if one adjacent vertex has type $\tau_{1}$ and the other has type $\tau_{2}$. The type of $v$ in $\tau_{5}$ if $v \sim_{G} v_{2}, v$ has valence two in $B(n)$, and the two adjacent vertices to $v$ is $\partial B(n)$ are $G$-equivalent to $v_{2}$. The vertex $v$ is of type $\tau_{6}$ if $v \sim_{G} v_{2}, v$ has valence 3 in $B(n)$, and one of the adjacent vertices has type $\tau_{1}$. Finally, $v$ is of type $\tau_{7}$ if $v \sim_{G} v_{2}, v$ has valence 4 in $B(n)$, and the two vertices adjacent to it in $\partial B(n)$ have type $\tau_{1}$. Let $B(n)^{\prime}$ be $B(n) \cup\left\{g T:|g|=n+1\right.$ and $g T \cap B(n)$ contains a vertex of type $\left.\tau_{2}\right\}$. It is now straightforward to show by induction that if $n$ is a positive integer then $B(n)$ is homeomorphic to a ball, $B(n)^{\prime}$ is convex, and each vertex of $\partial B(n)$ has one of the above seven types. The weight vector $U=\left[\begin{array}{lllllll}1 & 2 & 1 & 1 & 1 & 2 & 3\end{array}\right]$, and 
the recursion matrix and initial vector are

$$
A=\left[\begin{array}{ccccccc}
0 & 0 & p-2 & p-2 & p-3 & p-3 & p-3 \\
0 & 0 & 0 & 0 & 1 & 1 / 2 & 0 \\
0 & 0 & p-3 & p-3 & p-4 & p-4 & p-4 \\
0 & 0 & 0 & 0 & 2 & 1 & 0 \\
0 & 0 & 0 & 0 & 0 & 0 & 0 \\
1 & 0 & 1 & 1 / 2 & 0 & 1 / 2 & 1 \\
0 & 1 / 3 & 0 & 1 / 3 & 0 & 0 & 0
\end{array}\right] \quad \text { and } \quad V=\left[\begin{array}{c}
2 p-4 \\
1 \\
2 p-6 \\
2 \\
1 \\
2 \\
0
\end{array}\right]
$$

The growth function

$$
f(z)=\frac{z^{4}+(3 p+1) z^{3}+(4 p-4) z^{2}+(3 p+1) z+1}{z^{4}+(2-p) z^{3}+(4-p) z^{2}+(2-p) z+1}
$$

The last three cases are different from the first four because of the presence of buried domains (see Figure 5). As for the previous case, since the combinatorial balls are not convex the data for the types has to include more information about the adjacent vertices. We next analyze case $(v)$, where the angle measures of vertices $v_{1}, v_{2}$, and $v_{3}$ are $\pi / 2, \pi / p$, and $\pi / q$ (respectively), with $p, q \geq 4$. As for case (i), we can assume that $v_{2}$ and $v_{3}$ are in distinct $G$-orbits. Let $n$ be a positive integer such that $B(n)$ is homeomorphic to a ball, and let $w$ be a vertex of $\partial B(n)$. The vertex $w$ is of type $\tau_{1}\left(\tau_{2}\right)$ if $w \sim_{G} v_{1}, w$ has valence 2 in $B(n)$, and the two vertices adjacent to $w$ in $\partial B(n)$ are $G$-equivalent to $v_{2}\left(v_{3}\right)$. The vertex $w$ is of type $\tau_{3}$ if $w \sim_{G} v_{1}, w$ has valence 3 in $B(n)$, and the two adjacent vertices each have valence 2 in $B(n)$. If $w \sim_{G} v_{2}$ and $w$ has valence 2 in $B(n)$, then $w$ has type $\tau_{4}$ if the two vertices adjacent to $w$ in $\partial B(n)$ have type $\tau_{1}$, and $w$ has type $\tau_{5}$ if one adjacent vertex has type $\tau_{1}$ and the other has type $\tau_{3}$. The vertex $w$ is of type $\tau_{6}$ if $w \sim_{G} v_{2}$, $w$ has valence 3 in $B(n)$, and one of the adjacent vertices to $w$ has type $\tau_{1}$. The vertex $w$ is of type $\tau_{7}$ if $w \sim_{G} v_{2}, w$ has valence 4 in $B(n)$, and both adjacent vertices to $w$ in $\partial B(n)$ have type $\tau_{1}$. If $w \sim_{G} v_{3}$ and $w$ has valence 2 in $B(n)$, then $w$ has type $\tau_{8}$ if the two vertices adjacent to $w$ in $\partial B(n)$ have type $\tau_{2}$, and $w$ has type $\tau_{9}$ if one adjacent vertex has type $\tau_{2}$ and the other has type $\tau_{3}$. The vertex $w$ is of type $\tau_{10}$ if $w \sim_{G} v_{3}, w$ has valence 3 in $B(n)$, and one of the adjacent vertices to $w$ has type $\tau_{2}$. The vertex $w$ is of type $\tau_{11}$ if $w \sim_{G} v_{3}, w$ has valence 4 in $B(n)$, and both adjacent vertices to $w$ in $\partial B(n)$ have type $\tau_{2}$. Let $B(n)^{\prime}=B(n) \cup\{g D: g \in$ $G,|g|=n+1$, and $g D \cap B(n)$ contains a vertex of type $\left.\tau_{3}\right\}$. Using Lemma 4.1 (with $B(n)^{\prime}$ in the place of $B$ ), one can show inductively that each $B(n)^{\prime}$ is convex, each $B(n)$ is homeomorphic to a ball, and each vertex of $\partial B(n)$ has valence 2,3 , or 4 and has one of the above 11 types. Note that if $g \in G$ with $|g|=n$ and $g D \subset B(n-1)^{\prime}$, then $g D \subset \operatorname{int}(B(n))$ (these are the buried domains). To compute the growth series, we create a new type, $\tau_{12}$, which corresponds to a buried domain and not to a vertex of $\partial B(n)$. The weight 
vector $U=\left[\begin{array}{llllllllllll}1 & 1 & 2 & 1 & 1 & 2 & 3 & 1 & 1 & 2 & 3 & 1\end{array}\right]$, the recursion matrix

$$
A=\left[\begin{array}{ccccccccccccc}
0 & 0 & 0 & 0 & 0 & 0 & 0 & q-2 & q-3 & q-3 & q-3 & 0 \\
0 & 0 & 0 & p-2 & p-3 & p-3 & p-3 & 0 & 0 & 0 & 0 & 0 \\
0 & 0 & 0 & 0 & 1 / 2 & 1 / 2 & 0 & 0 & 1 / 2 & 1 / 2 & 0 & 0 \\
0 & 0 & 0 & 0 & 0 & 0 & 0 & q-3 & q-4 & q-4 & q-4 & 0 \\
0 & 0 & 0 & 0 & 0 & 0 & 0 & 0 & 1 & 1 & 0 & 0 \\
0 & 0 & 0 & 0 & 0 & 0 & 0 & 0 & 0 & 0 & 0 & 0 \\
0 & 1 / 3 & 0 & 0 & 0 & 0 & 0 & 2 / 3 & 1 / 3 & 1 / 3 & 2 / 3 & 0 \\
0 & 0 & 0 & p-3 & p-4 & p-4 & p-4 & 0 & 0 & 0 & 0 & 0 \\
0 & 0 & 0 & 0 & 1 & 1 & 0 & 0 & 0 & 0 & 0 & 0 \\
0 & 0 & 0 & 0 & 0 & 0 & 0 & 0 & 0 & 0 & 0 & 0 \\
1 / 3 & 0 & 0 & 2 / 3 & 1 / 3 & 1 / 3 & 2 / 3 & 0 & 0 & 0 & 0 & 0 \\
0 & 0 & 1 & 0 & 0 & 0 & 0 & 0 & 0 & 0 & 0 & 0
\end{array}\right]
$$

and the transpose of the initial vector $V$ is

$$
\left[\begin{array}{ccccccccccccc}
q-2 & p-2 & 1 & q-3 & 1 & 1 & 0 & p-3 & 1 & 1 & 0 & 0
\end{array}\right]
$$

The growth function

$$
f(z)=\frac{-z^{6}-2 z^{5}+(p q-2 s+3) z^{4}+(2 p q-2 s+4) z^{3}+(3 p q-2 s-3) z^{2}+(2 s-2) z+1}{z^{4}+(-p q+2 s-2) z^{2}+1},
$$

where $s=p+q$.

We next do case (vi), where the angle measures of the vertices $v_{1}, v_{2}$, and $v_{3}$ are $\pi / 2, \pi / 3$, and $\pi / q$ (respectively), with $q \geq 6$. We assume as before that $v_{2}$ and $v_{3}$ are in distinct $G$-orbits. As for case $(\mathrm{v})$, a vertex $w$ of $\partial B(n)$ can have angle measure greater than $\pi$ in $B(n)$ if it is $G$-equivalent to $v_{1}$ and has valence 3 in $B(n)$. However, in this case it can also have angle sum greater than $\pi$ in $B(n)$ if it is $G$-equivalent to $v_{2}$ and has valence 4 in $B(n)$. If one computed the recursion matrix and initial vector as for the previous case, then the type of a vertex would not be determined solely by the data from the vertex and its two adjacent vertices. In order not to have to use more information to determine the type, for this case we change conventions in how we compute the recursion matrix. Given a type $\tau_{j}$ corresponding to a vertex and not to a buried domain, let $v$ be a representative vertex of $\tau$, and let $n$ be a natural number such that $v \in \partial B(n)$. If $v$ has valence 4 , then the recursion matrix $A$ has entry $a_{i j}=b_{i j} / m(i)$, where $b_{i j}$ is the number of elements of $G$ of length $n+1$ that have type $\tau_{i}$ and are connected by an edge to $v$, and if $b_{i j} \neq 0$ then $m(i)$ is the number of vertices of $\partial B(n)$ that have valence 4 and are connected by an edge to a vertex $w \in \partial B(n+1)$ with type $\tau_{i}$. If $v$ does not have valence 4, then the recursion matrix $A$ has entry $a_{i j}=b_{i j} / m(i)$, where $b_{i j}$ is the number of elements of $G$ of length $n+1$ that have type $\tau_{i}$, are connected by an edge to $v$, and are not connected by an edge to a vertex in $\partial B(n)$ that has valence 4 , and $m(i)$ is the number of vertices of $\partial B(n)$ that are connected by an edge to a vertex $w \in \partial B(n+1)$ with type $\tau_{i}$. That is, vertices of valence 4 count all of their output.

Let $n$ be a positive integer such that $B(n)$ is homeomorphic to a ball, let $w$ be a vertex of $\partial B(n)$, let $w_{1}$ and $w_{2}$ be the two adjacent vertices to $w$ in $\partial B(n)$, and let $x_{1}$ and $x_{2}$ be the two vertices adjacent to $w_{1}$ and $w_{2}$ (respectively) besides $w$. If $w \sim_{G} v_{1}, w$ has valence 2 in $B(n), w_{1}$, and $w_{2}$ are $G$-equivalent to $v_{2}$, and $w_{1}$ and $x_{1}$ have valence 2 in $B(n)$, then $w$ has type $\tau_{1}$ if $w_{2}$ and $x_{2}$ have valence 2 in $B(n)$, type $\tau_{2}$ if $w_{2}$ has valence 2 in 
$B(n)$ and $x_{2}$ has valence 3 in $B(n)$, type $\tau_{3}$ if $w_{2}$ has valence 3 in $B(n)$, and type $\tau_{4}$ if $w_{2}$ has valence 4 in $B(n)$ and $x_{2}$ has valence 2 in $B(n)$. If $w$ is $G$-equivalent to $v_{1}$ and $w_{1}$ and $w_{2}$ are $G$-equivalent to $v_{3}$, then $w$ has type $\tau_{5}$. If $w$ is $G$-equivalent to $v_{1}, w$ has valence 3 in $B(n)$, and $w_{1}$ and $w_{2}$ each have valence 2 in $B(n)$, then $w$ has type $\tau_{6}$. If $w$ is $G$-equivalent to $v_{2}, w$ has valence 2 in $B(n)$, and $w_{1}$ and $w_{2}$ are $G$-equivalent to $v_{1}$, then $w$ has type $\tau_{7}$ if $w_{1}$ and $w_{2}$ each have valence 2 in $B(n)$ and type $\tau_{8}$ if one of $w_{1}$ and $w_{2}$ has valence 2 in $B(n)$ and the other has valence 3 in $B(n)$. The vertex $w$ has type $\tau_{9}$ if $w$ is $G$-equivalent to $v_{2}, w$ has valence 3 in $B(n), w_{1}$ has valence 2 and is $G$-equivalent to $v_{1}$, and $w_{2}$ has valence 3 in $B(n)$ and is $G$-equivalent to $v_{3}$. The type of $w$ is $\tau_{10}$ if $w$ is $G$-equivalent to $v_{2}, w$ has valence 4 in $B(n)$, and $w_{1}$ and $w_{2}$ are $G$-equivalent to $v_{1}$ and have valence 2 in $B(n)$. If $w$ is $G$-equivalent to $v_{3}, w_{1}$ is $G$-equivalent to $v_{1}$ and has valence 2 in $B(n)$, and $w_{2}$ is $G$-equivalent to $v_{1}$ and has valence 3 in $B(n)$, then $w$ has type $\tau_{11}$ if it has valence 2 in $B(n)$ and $\tau_{14}$ if it has valence 4 in $B(n)$. The type of $v$ is $\tau_{12}$ if $w \sim_{G} v_{3}, w$ has valence 3 in $B(n), w_{1} \sim_{G} v_{1}, w_{1}$ has valence 2 in $B(n), w_{2} \sim_{G} v_{2}$, and $w_{2}$ has valence 3 in $B(n)$. If $w \sim_{G} v_{3}, w_{1}$ and $w_{2}$ are $G$-equivalent to $v_{1}$, and $w_{1}$ and $w_{2}$ have valence 2 in $B(n)$, then $w$ has type $\tau_{13}$ if it has valence 4 in $B(n)$ and type $\tau_{15}$ if it has valence 6 in $B(n)$. As for the previous case, there is one more type, $\tau_{16}$, that corresponds to a buried domain and not to a vertex of $\partial B(n)$. One can show inductively that each $B(n)$ is homeomorphic to a ball and that there is a linear recursion with the above types. The weight vector $U=$ $\left[\begin{array}{llllllllllllllll}1 & 1 & 1 & 1 & 1 & 2 & 1 & 1 & 2 & 3 & 1 & 2 & 3 & 3 & 5 & 1\end{array}\right]$, the recursion matrix is

$$
\left[\begin{array}{ccccccccccccccccc}
0 & 0 & 0 & 0 & 0 & 0 & 0 & 0 & 0 & 0 & q-5 & q-5 & q-5 & q-6 & q-6 & 0 \\
0 & 0 & 0 & 0 & 0 & 0 & 0 & 0 & 0 & 0 & 1 & 1 & 0 & 1 & 0 & 0 \\
0 & 0 & 0 & 0 & 0 & 0 & 0 & 0 & 0 & 0 & 0 & 0 & 0 & 0 & 0 & 0 \\
0 & 0 & 0 & 0 & 0 & 0 & 0 & 0 & 0 & 0 & 1 & 1 & 2 & 1 & 2 & 0 \\
0 & 0 & 0 & 0 & 0 & 0 & 1 & 0 & 0 & 0 & 0 & 0 & 0 & 0 & 0 & 0 \\
0 & 0 & 0 & 0 & 0 & 0 & 0 & 1 / 2 & 1 / 2 & 0 & 1 / 2 & 1 / 2 & 0 & 1 / 2 & 0 & 0 \\
0 & 0 & 0 & 0 & 0 & 0 & 0 & 0 & 0 & 0 & q-4 & q-4 & q-4 & q-5 & q-5 & 0 \\
0 & 0 & 0 & 0 & 0 & 0 & 0 & 0 & 0 & 0 & 1 & 1 & 0 & 1 & 0 & 0 \\
0 & 0 & 0 & 0 & 0 & 0 & 0 & 0 & 0 & 0 & 0 & 0 & 0 & 0 & 0 & 0 \\
0 & 0 & 0 & 0 & 1 & 0 & 0 & 0 & 0 & 0 & 0 & 0 & 0 & 0 & 0 & 0 \\
0 & 0 & 0 & 0 & 0 & 0 & 0 & 0 & 0 & 0 & 0 & 0 & 0 & 0 & 0 & 0 \\
0 & 0 & 0 & 0 & 0 & 0 & 0 & 0 & 0 & 0 & 0 & 0 & 0 & 0 & 0 & 0 \\
1 & 0 & 0 & 0 & 0 & 0 & 0 & 0 & 0 & 0 & 0 & 0 & 0 & 0 & 0 & 0 \\
0 & 1 & 1 & 0 & 0 & 0 & 0 & 0 & 0 & 0 & 0 & 0 & 0 & 0 & 0 & 0 \\
0 & 0 & 0 & 1 / 2 & 0 & 0 & 0 & 0 & 0 & 0 & 0 & 0 & 0 & 0 & 0 & 0 \\
0 & 0 & 0 & 0 & 0 & 1 & 0 & 0 & 0 & 0 & 0 & 0 & 0 & 0 & 0 & 0
\end{array}\right]
$$

and the transpose of the initial vector is

$$
\left[\begin{array}{lllllllllllllllll}
q-4 & 1 & 1 & 0 & 1 & 1 & q-3 & 1 & 1 & 0 & 1 & 1 & 0 & 0 & 0 & 0
\end{array}\right]
$$

The growth function

$$
f(z)=\frac{-z^{6}-2 z^{5}+(q-3) z^{4}+(4 q-2) z^{3}+(7 q-9) z^{2}+(2 q+4) z+1}{z^{4}+(4-q) z^{2}+1} .
$$

Finally, we analyze case (vii), where $v_{1}$ has valence 3 and $v_{2}$ and $v_{3}$ have valence $2 p, p \geq 6$. Let $n$ be a positive integer such that $B(n)$ is homeomorphic to a ball, and let $v$ be a vertex of $\partial B(n)$. Then $v$ has type $\tau_{1}$ if $v \sim_{G} v_{1}$ and $v$ has valence 2 in $B(n)$, type $\tau_{2}$ if $v \sim_{G} v_{2}$ and $v$ has valence 
2 in $B(n)$, and type $\tau_{3}$ if $v \sim_{G} v_{2}$ and $v$ has valence 4 in $B(n)$. For each natural number $n$, let $B(n)^{\prime}=B(n) \cup\{g D: g \in G,|g|=n+1$ and $g D \cap B(n)$ is a union of two edges $\}$. One can show inductively that each $B(n)^{\prime}$ is convex, each $B(n)$ is homeomorphic to a ball, and each vertex of $\partial B(n)$ has one of the above three types. There is a fourth type, $\tau_{4}$, which corresponds to a buried domain and not to a vertex. There are buried domains in $B(n)^{\prime}$ and buried domains in $B(n+1)-\operatorname{int}\left(B(n)^{\prime}\right)$. One can show by induction that each $B(n)$ is homeomorphic to a ball and that there is a linear recursion with the above types. The weight vector $U=\left[\begin{array}{llll}1 & 1 & 3 & 1\end{array}\right]$, and the recusion matrix and initial vector are

$$
A=\left[\begin{array}{cccc}
0 & p-4 & p-5 & 0 \\
0 & p-5 & p-6 & 0 \\
0 & 1 & 1 & 0 \\
2 & 0 & 0 & 0
\end{array}\right] \text { and } V=\left[\begin{array}{c}
2 p-4 \\
2 p-6 \\
2 \\
1
\end{array}\right]
$$

The growth function

$$
f(z)=\frac{-3 z^{3}+(3 p+1) z^{2}+(3 p+1) z+1}{z^{2}+(4-p) z+1} .
$$

\section{THE EXCEPTIONAL QUADRANGULAR DOMAINS}

In this section, we consider the cases where $D$ has four sides and a vertex with valence 3 . We will see that these are exactly the cases for which $D$ has four sides and there are either buried domains or positive integers $n$ and vertices in $\partial B(n)$ with valence 3 in $B(n)$. A domain $g D$ with $|g|=n$ is buried if $g D \subset \operatorname{int}(B(n))$. This will occur if $g D \cap B(n-1)$ contains a vertex $v$ whose valence in $B(n)$ is one less than its valence in the tessellation, and such that the vertex of $g D$ which is not in $B(n-1)$ has valence 3 in the tessellation.

Suppose that $D$ has four sides, and let $v_{1}, v_{2}, v_{3}$, and $v_{4}$ denote the vertices of $D$, chosen so that $v_{1}$ and $v_{3}$ are not adjacent (so the vertices are in clockwise or counterclockwise order). We will assume that the vertex $v_{1}$ has valence 3. Then $G$ contains an element of order 3 that fixes $v_{1}$. As before, we will assume that the angle measure of a vertex of $D$ is $2 \pi$ divided by its valence. If $v_{3}$ is fixed by an element of $G$ of order $q, q>2$, then the vertices $v_{2}$ and $v_{4}$ each have angle measure $\pi / r$ and $G$ is the triangle group $\Delta(3, q, r)$. In this case $1 / q+1 / r \leq 2 / 3$. If $v_{3}$ is not $G$-equivalent to $v_{2}$ and $v_{4}$, then the tessellation is determined by $G$ and the growth function is the same as the growth function for a triangle group $\Delta(3, q, r)$.

We first compute the growth function for the triangle group $\Delta(3, q, r)$ with a four-sided fundamental domain as described above. We will do this by cases as follows:

(i) $q \geq 5$ and $r \geq 3$; and

(ii) $q=3$ and $r \geq 3$;

(iii) $q=4$ and $r \geq 3$; and

(iv) $q \geq 6$ and $r=2$.

We start with an analogue of Lemma 1.2.

Lemma 5.1. Let $q, r$ be positive integers with $r \geq 3, q=3$, or $q \geq 5$, and $q \neq 3$ if $r=3$, let $G=\Delta(3, q, r)$, and let $D$ be a fundamental domain 
for the action of $G$ as described above. The following conditions hold for every nonnegative integer $n$.

(i) $B(n)$ is homeomorphic to a ball.

(ii) If $g \in G$ with $|g|=n$, then $g D \cap B(n-1)$ is connected.

(iii) If $g, h \in G$ with $|g|=|h|=n, g D \cap h D \neq \varnothing$, and $g D \cap h D \cap$ $B(n-1)=\varnothing$, then there is an element $g^{\prime} \in G$ with $\left|g^{\prime}\right|=n$ and $\left(g D \cup h D \cup g^{\prime} D\right) \cap B(n-1)$ connected.

(iv) $A$ vertex $v \in \partial B(n)$ that is not G-equivalent to $v_{3}$ has valence 1 or 2 . $A$ vertex $v \in \partial B(n)$ that is G-equivalent to $v_{3}$ has valence 1,2 or (if $q>3) 3$.

Proof. The case $n=0$ is clear, so assume that $n \geq 0$ and that (i)-(iv) hold for $n$. Let $\theta$ be the minimum measure of an angle of $D$, let $K=0$ if $X=E^{2}$, and let $K=1$ if $X=H^{2}$. Let $g \in G$ with $|g|=n+1$. We prove by contradiction that $g D \cap B(n)$ is connected. Suppose not. Then there is a polygon $C$ such that $\operatorname{int}(C)$ is a component of $X-(g D \cup B(n))$. Let $w_{1}$ and $w_{2}$ be the vertices of $C \cap g D \cap B(n)$. Let $s$ be the number of edges of $\partial C$ which are in $g D$ and let $t$ be the number of edges of $\partial C$ which are in $B(n) . \quad \partial C$ has $s+t$ vertices. The two vertices of $\partial C$ in $g D \cap B(n)$ each have angle measure at least $\theta$, and have angle measure at least $\pi / r$ if they are $G$-equivalent to $v_{2}$. The $s-1$ vertices in $g D-B(n)$ each have angle measure at least $4 \pi / 3$. Since a vertex of $\partial B(n)$ which is $G$-equivalent to $v_{1}$ or $v_{3}$ is adjacent to vertices that are $G$-equivalent to $v_{2}$, by (iv) the sum of the angle measure of the $t-1$ vertices of $C$ in $B(n)-g D$ is at least

$$
\frac{t-2}{2}\left(\frac{2 \pi}{3}+\frac{(2 r-2) \pi}{r}\right)+\pi \frac{2}{3}=(t-2) \pi \frac{4 r-3}{3 r}+\pi \frac{2}{3}
$$

if $w_{1}$ and $w_{2}$ are $G$-equivalent to $v_{2}$ and is at least

$$
\frac{t-2}{2}\left(\frac{2 \pi}{3}+\frac{(2 r-2) \pi}{r}\right)
$$

otherwise. By the Gauss-Bonnet formula, if $w_{1}$ and $w_{2}$ are both $G$-equivalent to $v_{2}$ then

$$
\begin{aligned}
K \cdot \operatorname{area}(C) & =\pi(s+t-2)-\text { angle sum of vertices of } C \\
& \leq \pi(s+t-2)-\frac{(s-1) 4 \pi}{3}-(t-2) \pi \frac{4 r-3}{3 r}-\frac{2 \pi}{3}-\frac{2 \pi}{r} \\
& =\pi\left(-\frac{s}{3}-\frac{t}{3}+\frac{t}{r}+\frac{4}{3}-\frac{4}{r}\right),
\end{aligned}
$$

and if $w_{1}$ or $w_{2}$ is not $G$-equivalent to $v_{2}$ then

$$
\begin{aligned}
K \cdot \operatorname{area}(C) & \leq \pi(s+t-2)-\frac{(s-1) 4 \pi}{3}-(t-1) \pi \frac{4 r-3}{3 r}-2 \theta \\
& =\pi\left(-\frac{s}{3}-\frac{t-1}{3}+\frac{t-1}{r}+\frac{1}{3}-\frac{2 \theta}{\pi}\right) .
\end{aligned}
$$

Since $s+t \geq 4$, the right-hand side of both inequalities is negative. This gives a contradiction, so $g D \cap B(n)$ is connected and (ii) holds for $n+1$.

Now suppose that $g, h \in G$ with $|g|=|h|=n+1, g \neq h$, and $g D \cap$ $h D \neq \varnothing$. Suppose that $g D \cap h D$ does not contain a vertex of $\partial B(n)$ and that 
there is no element $g^{\prime} \in G$ with $\left|g^{\prime}\right|=n+1$ and $\left(g D \cup h D \cup g^{\prime} D\right) \cap B(n)$ connected. Then there is a polygon $C$ such that $\operatorname{int}(C)$ is a component of $X-(g D \cup h D \cup B(n))$ and area $(C) \geq 2 \cdot \operatorname{area}(D)$. Let $s_{1}$ be the number of edges of $C$ in $g D$, let $s_{2}$ be the number of edges of $C$ in $h D$, and let $t$ be the number of edges of $C$ in $B(n)$. Then $s_{1}>0, s_{2}>0$, and $t>0$. Since $D$ has only four sides, we can assume that $s_{1} \leq 2$ and $s_{2} \leq 2$. Let $w_{1}$ be the vertex $C \cap g D \cap B(n)$, let $w_{2}=C \cap h D \cap B(n)$, and let $w_{3}$ be the vertex $C \cap g D \cap h D$. Let $\alpha$ be the angle measure of $w_{3}$ in $C$.

First suppose that $w_{1}$ and $w_{2}$ are both $G$-equivalent to $v_{2}$. Then

$$
\begin{aligned}
K \cdot \operatorname{area}(C) \leq & \pi\left(s_{1}+s_{2}+t-2\right)-\frac{\left(s_{1}+s_{2}-2\right) 4 \pi}{3} \\
& -(t-2) \pi \frac{4 r-3}{3 r}-\frac{2 \pi}{3}-\frac{2 \pi}{r}-\alpha \\
= & \pi\left(-\frac{s_{1}+s_{2}}{3}-\frac{t}{3}+\frac{t}{r}+\frac{8}{3}-\frac{4}{r}-\frac{\alpha}{\pi}\right) .
\end{aligned}
$$

Since $\operatorname{area}(C) \geq 2 \cdot \operatorname{area}(D) \geq 2 \pi(2-2 / 3-2 / r-2 / q)$,

$$
-\frac{s_{1}+s_{2}}{3}-\frac{t}{3}+\frac{t}{r}-\frac{\alpha}{\pi}+\frac{4}{q} \geq 0 .
$$

Every edge in the tessellation connects a vertex $G$-equivalent to $v_{2}$ to a vertex that is not $G$-equivalent to $v_{2}$, so $s_{1}=s_{2}=2$ or $s_{1}=s_{2}=1$. We get a contradiction if $s_{1}=s_{2}=2$. If $s_{1}=s_{2}=1$, then $\alpha \geq 2 \pi / q$ and the inequality has no solution.

Now suppose that $w_{1}$ is $G$-equivalent to $v_{2}$ and $w_{2}$ is not $G$-equivalent to $v_{2}$. Then $s_{1} \neq s_{2}$, so $s_{1}+s_{2}=3$ and

$$
\begin{aligned}
K \cdot \operatorname{area}(C) & \leq \pi(t+1)-\frac{4 \pi}{3}-(t-1) \pi \frac{4 r-3}{3 r}-\frac{2 \pi}{q}-\frac{\pi}{r}-\theta \\
& =\pi\left(1-\frac{t}{3}+\frac{t}{r}-\frac{2}{r}-\frac{2}{q}-\frac{\theta}{\pi}\right) .
\end{aligned}
$$

Since $\operatorname{area}(C) \geq 2 \cdot \operatorname{area}(D)$,

$$
-\frac{t}{3}+\frac{t}{r}-\frac{5}{3}+\frac{2}{r}+\frac{2}{q}-\frac{\theta}{\pi} \geq 0 .
$$

This is impossible.

Finally, suppose that $w_{1}$ is not $G$-equivalent to $v_{2}$ and $w_{2}$ is not $G$ equivalent to $v_{2}$. Then

$$
\begin{aligned}
K \cdot \operatorname{area}(C) & \leq \pi\left(s_{1}+s_{2}+t-2\right)-\frac{\left(s_{1}+s_{2}-2\right) 4 \pi}{3}-(t-1) \pi \frac{4 r-3}{3 r}-\frac{4 \pi}{q}-\theta \\
& =\pi\left(-\frac{s_{1}+s_{2}}{3}-\frac{t}{3}+\frac{t}{r}+2-\frac{1}{r}-\frac{4}{q}-\frac{\alpha}{\pi}\right) .
\end{aligned}
$$

Since $\operatorname{area}(C) \geq 2 \cdot \operatorname{area}(D)$,

$$
-\frac{s_{1}+s_{2}}{3}-\frac{t}{3}+\frac{t}{r}-\frac{2}{3}+\frac{3}{r}-\frac{\alpha}{\pi} \geq 0 .
$$

This is also impossible, and so (iii) holds for $B(n+1)$.

If $g, h \in G$ with $|g|=n+1=|h|$ and $g D \cap h D$ is nonempty and does not contain a point of $\partial B(n)$, then the element $g^{\prime}$ with $\left(g D \cup g^{\prime} D \cap h D\right) \cap B(n)$ 
connected must have $g^{\prime} D \cap B(n)$ a union of two edges whose common vertex has valence 2 in $B(n)$ and angle measure $4 \pi / 3$ in $B(n)$. This will correspond to a buried domain exactly if $q=3$. Condition (iv) for $B(n+1)$ now follows from (iii). Let $h_{1}, \ldots, h_{m}$ be a listing of the elements $h$ of $G$ of length $n+1$ such that $h D \cap B(n)$ contains a vertex of $\partial B(n)$ with valence 2 in $B(n)$ and angle measure $4 \pi / 3$ in $B(n)$, and let $h_{m+1}, \ldots, h_{k}$ be a listing of the other elements of length $n+1$. Then for each $j \in\{1, \ldots, k\}, B(n) \cup\left\{h_{i} D: i \in\{1, \ldots, j\}\right\}$ is homeomorphic to a ball. Thus $B(n+1)$ is homeomorphic to a ball and the proof is complete.

Now suppose that $r \geq 3$ and $q \geq 5$. Then some of the vertices of $\partial B(n)$ that are $G$-equivalent to $v_{1}$ will have valence 1 and some will have valence 2, and the type data of the adjacent vertices need to take this valence into account. Let $n \in \mathbb{Z}$ with $n \geq 1$, and let $v$ be a vertex of $\partial B(n)$. If $v \sim_{G} v_{1}$, then $v$ has type $\tau_{1}\left(\tau_{2}\right)$ if it has valence $1(2)$ in $B(n)$. If $v \sim_{G} v_{3}$, then $v$ has type $\tau_{3}\left(\tau_{4}\right)$ if it has valence $2(3)$ in $B(n)$. If $v \sim_{G} v_{2}$, then $v$ has type $\tau_{5}$ if it has valence 1 in $B(n)$ and one of the adjacent vertices to $v$ in $\partial B(n)$ has type $\tau_{2}$, and $v$ has type $\tau_{6}$ if it has valence 2 in $B(n)$ and the two adjacent vertices in $\partial B(n)$ are $G$-equivalent to $v_{3}$. Suppose $v \sim_{G} v_{2}$, the valence of $v$ in $\partial B(n)$ is 2 , and the two vertices, $w_{1}$ and $w_{2}$, that are adjacent to $v$ in $\partial B(n)$ are each $G$-equivalent to $v_{1}$. The type of $v$ is $\tau_{7}$ if $w_{1}$ and $w_{2}$ both have type $\tau_{1}, \tau_{8}$ if one of the adjacent vertices has type $\tau_{1}$ and the other has type $\tau_{2}$, and type $\tau_{9}$ if both $w_{1}$ and $w_{2}$ have type $\tau_{2}$. If $w$ is a vertex of $\partial B(n+1)$ and $w$ has valence 1 in $B(n+1)$, then there is a unique element $g \in G$ with $|g|=n+1$ and $w \subset g D$. Then $g D \cap B(n)$ is a vertex, and we say that $w$ is in the output of that vertex. If $w$ has valence at least 2 in $B(n+1)$, then $w$ will be connected by edges to at least one vertex of $\partial B(n)$. We will say that $w$ is in the output of each such vertex $v \subset \partial B(n)$, and we will count their contributions equally in producing $w$. Vertices of types $\tau_{8}$ and $\tau_{9}$ arise as part of the output of vertices of types $\tau_{3}$ and $\tau_{4}$. If $q=5$, then the output of a vertex of type $\tau_{4}$ has type $\tau_{9}$. If $q \geq 6$, then the output of a vertex of type $\tau_{4}$ is $q-5$ vertices of type $\tau_{1}, q-6$ vertices of type $\tau_{7}$, and two vertices of type $\tau_{8}$, and type $\tau_{9}$ does not occur. There is a linear recursion with these nine types. If $g \in G$ with $|g|=n$, then either $g D$ contains a single vertex in $\partial B(n)$ and it has type $\tau_{4}$, or $g D \cap \partial B(n)$ contains exactly 2 vertices with valence 2 in $B(n)$. Since a vertex $v \subset B(n)$ with valence two in $B(n)$ will be in the boundary of two domains $g D$ with $|g|=n$, one can use weight vector $U=\left[\begin{array}{lllllllll}0 & 1 & 1 & 2 & 0 & 1 & 1 & 1 & 1\end{array}\right]$. If $q=5$, then the recursion matrix and initial vector are

$$
\left[\begin{array}{ccccccccc}
0 & 0 & 1 & 0 & 0 & 0 & 0 & 0 & 0 \\
0 & 0 & 0 & 0 & r-1 & r-1 & r-2 & r-2 & r-2 \\
0 & 0 & 0 & 0 & r-2 & r-2 & r-1 & r-2 & r-3 \\
0 & 0 & 0 & 0 & 1 / 2 & 0 & 0 & 1 / 2 & 1 \\
0 & 0 & 0 & 0 & 2 r-3 & 2 r-4 & 2 r-4 & 2 r-4 & 2 r-4 \\
1 & 0 & 0 & 0 & 0 & 0 & 0 & 0 & 0 \\
0 & 0 & 0 & 0 & 0 & 0 & 0 & 0 & 0 \\
0 & 0 & 2 & 0 & 0 & 0 & 0 & 0 & 0 \\
0 & 0 & 0 & 1 & 0 & 0 & 0 & 0 & 0
\end{array}\right] \text { and }\left[\begin{array}{c}
2 \\
2 r-2 \\
2 r-2 \\
0 \\
4 r-6 \\
1 \\
1 \\
2 \\
0
\end{array}\right]
$$




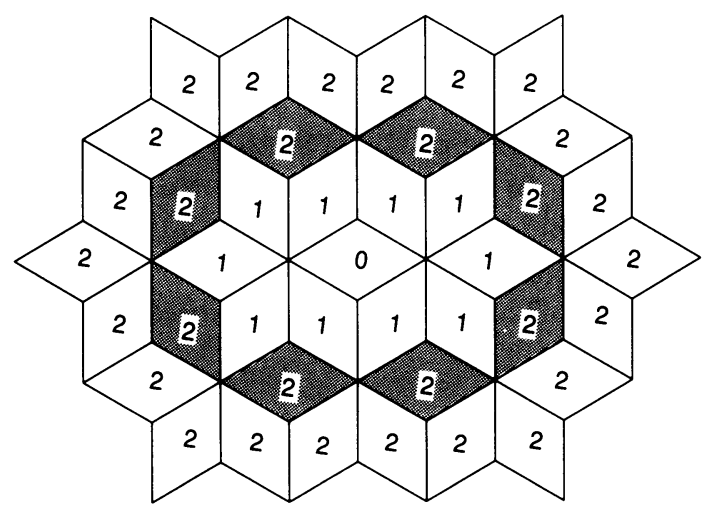

FIGURE 6. $\Delta(3,3,3)$

If $q \geq 6$, then the recursion matrix and initial vector are

$$
\left[\begin{array}{ccccccccc}
0 & 0 & q-4 & q-5 & 0 & 0 & 0 & 0 & 0 \\
0 & 0 & 0 & 0 & r-1 & r-1 & r-2 & r-2 & r-2 \\
0 & 0 & 0 & 0 & r-2 & r-2 & r-1 & r-2 & r-3 \\
0 & 0 & 0 & 0 & 1 / 2 & 0 & 0 & 1 / 2 & 1 \\
0 & 0 & 0 & 0 & 2 r-3 & 2 r-4 & 2 r-4 & 2 r-4 & 2 r-4 \\
1 & 0 & 0 & 0 & 0 & 0 & 0 & 0 & 0 \\
0 & 0 & q-5 & q-6 & 0 & 0 & 0 & 0 & 0 \\
0 & 0 & 2 & 2 & 0 & 0 & 0 & 0 & 0 \\
0 & 0 & 0 & 0 & 0 & 0 & 0 & 0 & 0
\end{array}\right]
$$

and

$$
\left[\begin{array}{c}
q-3 \\
2 r-2 \\
2 r-2 \\
0 \\
4 r-6 \\
1 \\
q-4 \\
2 \\
0
\end{array}\right]
$$

In both cases, the growth function

$$
f(z)=\frac{z^{4}+(2 r+q-1) z^{3}+(q r-r+q) z^{2}+(2 r+q-1) z+1}{z^{4}+(4-2 r) z^{3}+(-q r+r+q+2) z^{2}+(4-2 r) z+1} .
$$

We next do case (ii) with $q=3$ (see Figure 6). There is a linear recursion, with three types, on the vertices of $\partial B(n), n \geq 1$. Let $n$ be a positive integer, and let $v$ be a vertex of $\partial B(n)$. The vertex $v$ is of type $\tau_{1}$ if $v$ is $G$-equivalent to $v_{1}$ or $v_{3}$ and has valence 2 in $B(n)$. If $v \sim_{G} v_{2}$, then $v$ has type $\tau_{2}$ if $v$ has valence 1 in $\partial B(n)$ and has type $\tau_{3}$ if $v$ has valence 2 in $\partial B(n)$. For counting purposes, there is a fourth type $\tau_{4}$ that corresponds to a buried domain. If $v$ has type $\tau_{1}$ and $g \in G$ with $|g|=n+1$ and $v \subset g D$, then $g D$ will be a buried domain. It is easy to see that $\partial B(1)$ contains $4 r-4$ vertices of type $\tau_{1}, 4 r-6$ vertices of type $\tau_{2}$, and two vertices of type $\tau_{3}$. One can guess 
at a linear recursion among these types by drawing the local pictures around a vertex in $\partial B(n)$ and ignoring the possibilities of interactions with vertices that are far away. The output of a vertex of type $\tau_{1}$ is a single element of type $\tau_{4}$ corresponding to the buried domain. If $v$ is a vertex of $\partial B(n)$ of type $\tau_{2}$, then there will be $2 r-1$ elements of $g \in G$ with $|g|=n+1$ and $v \subset g D$. Two of these will be buried domains coming from the adjacent vertices of type $\tau_{1}$. There will be two vertices of type $\tau_{3}$ coming from domains adjacent to the buried domains, but $v$ only accounts for producing $1 / 2$ of each of them. The other $2 r-5$ elements will each produce a single vertex of type $\tau_{2}$, and there will be $2 r-4$ vertices of type $\tau_{1}$. Similarly, a vertex $v$ of type $\tau_{3}$ would be expected to have output $2 r-5$ vertices of type $\tau_{1}, 2 r-6$ vertices of type $\tau_{2}$, and two vertices of type $\tau_{3}$. If $r>3$, then Lemma 5.1 implies that this gives a linear recursion. The problem if $r=3$ is to show that there are no further identifications in going from $B(n)$ to $B(n+1)$. The easiest way (following [5]) to show that this works is to recursively construct a space $Y$ such that $Y$ is the union of combinatorial balls constructed exactly according to the local data. Since $Y$ is simply connected (each combinatorial ball is homeomorphic to a ball) and each vertex has angle sum $2 \pi, Y$ is homeomorphic to $X$ and there is a linear recursion as described above. The weight vector $U=\left[\begin{array}{llll}1 & 0 & 1 & 1\end{array}\right]$, and the recursion matrix and initial vector are

$$
A=\left[\begin{array}{cccc}
0 & 2 r-4 & 2 r-5 & 0 \\
0 & 2 r-5 & 2 r-6 & 0 \\
0 & 1 & 1 & 0 \\
1 & 0 & 0 & 0
\end{array}\right] \text { and } V=\left[\begin{array}{c}
4 r-4 \\
4 r-6 \\
2 \\
0
\end{array}\right]
$$

The growth function

$$
f(z)=\frac{-2 z^{3}+(4 r-1) z^{2}+(2 r+2) z+1}{z^{2}+(4-2 r) z+1} .
$$

We next do case (iii), with $q=4$. As for the first case, some of the vertices of $\partial B(n)$ that are $G$-equivalent to $v_{1}$ will have valence 1 and some will have valence 2 , and the type data of the adjacent vertices need to take this valence into account. Let $n$ be a positive integer such that $B(n)$ is homeomorphic to a ball, and let $v$ be a vertex of $\partial B(n)$. If $v \sim_{G} v_{1}$, then $v$ has type $\tau_{1}\left(\tau_{2}\right)$ if it has valence $1(2)$ in $B(n)$. If $v \sim_{G} v_{3}$, then $v$ has type $\tau_{3}, \tau_{4}$, or $\tau_{5}$ if it has valence 1,2 , or 3 (respectively) in $B(n)$. If $v \sim_{G} v_{2}$, then $v$ has type $\tau_{6}$ if it has valence 1 in $B(n)$ and the adjacent vertices to $v$ in $\partial B(n)$ have types $\tau_{2}$ and $\tau_{4}$, and $v$ has type $\tau_{7}$ if it has valence one in $B(n)$ and the adjacent vertices to $v$ in $\partial B(n)$ have types $\tau_{2}$ and $\tau_{5}$, and $v$ has type $\tau_{8}$ if it has valence 2 in $B(n)$ and the two adjacent vertices in $\partial B(n)$ have types $\tau_{4}$. Suppose $v \sim_{G} v_{2}$, the valence of $v$ in $\partial B(n)$ is 2 , and the two vertices, $w_{1}$ and $w_{2}$, that are adjacent to $v$ in $\partial B(n)$ are each $G$-equivalent to $v_{1}$. The type of $v$ is $\tau_{9}$ if one of the adjacent vertices has type $\tau_{1}$ and the other has type $\tau_{2}$, and is type $\tau_{10}$ if both $w_{1}$ and $w_{2}$ have type $\tau_{2}$. Finally, there is a type $\tau_{11}$ that corresponds to a buried domain. This case was not covered by Lemma 5.1, and we leave it to the reader to show that one can recursively construct a space $Y$ from combinatorial balls by using only the local data such that each combinatorial ball in $Y$ is homeomorphic to a ball, there is a linear recursion on the vertices in $Y$ with the above types, and $Y$ is homeomorphic 
to $X$. The weight vector $U=\left[\begin{array}{lllllllllll}0 & 1 & 0 & 1 & 2 & 0 & 0 & 1 & 1 & 1 & 1\end{array}\right]$, and $A$ and $V$ are

$$
\left[\begin{array}{ccccccccccc}
0 & 0 & 1 & 0 & 0 & 0 & 0 & 0 & 0 & 0 & 0 \\
0 & 0 & 0 & 0 & 0 & r-1 & r-2 & r-1 & r-2 & r-2 & 0 \\
0 & 0 & 0 & 0 & 0 & 0 & 0 & 0 & 0 & 0 & 0 \\
0 & 0 & 0 & 0 & 0 & r-2 & r-2 & r-2 & r-2 & r-3 & 0 \\
0 & 0 & 0 & 0 & 0 & 1 / 2 & 1 / 2 & 0 & 1 / 2 & 1 & 0 \\
0 & 0 & 0 & 0 & 0 & 2 r-4 & 2 r-5 & 2 r-4 & 2 r-5 & 2 r-6 & 0 \\
0 & 0 & 0 & 0 & 0 & 1 & 1 & 0 & 1 & 2 & 0 \\
1 & 0 & 0 & 0 & 0 & 0 & 1 / 2 & 0 & 0 & 0 & 0 \\
0 & 0 & 2 & 0 & 0 & 0 & 0 & 0 & 0 & 0 & 0 \\
0 & 0 & 0 & 1 & 0 & 0 & 0 & 0 & 0 & 0 & 0 \\
0 & 0 & 0 & 0 & 1 & 0 & 0 & 0 & 0 & 0 & 0
\end{array}\right]
$$

and

$$
\left[\begin{array}{c}
1 \\
2 r-2 \\
0 \\
2 r-2 \\
0 \\
4 r-6 \\
0 \\
1 \\
2 \\
0 \\
0
\end{array}\right]
$$

The growth function

$$
f(z)=\frac{-z^{6}+(2 r-4) z^{5}+(3 r-3) z^{4}+(4 r+2) z^{3}+(r+3) z^{2}+(2 r+2) z+1}{z^{4}+(3-2 r) z^{3}+(4-r) z^{2}+(3-2 r) z+1} .
$$

The next case is (iv), where $r=2$ and $q \geq 6$. Let $n$ be a positive integer such that $B(n)$ is homeomorphic to a ball, let $w$ be a vertex of $\partial B(n)$, and let $w_{1}$ and $w_{2}$ be the adjacent vertices to $w$ in $\partial B(n)$. The type of $w$ is $\tau_{1}$ if $w \sim_{G} v_{1}, w$ has valence 1 in $B(n)$, and $w_{1}$ and $w_{2}$ are $G$-equivalent to $v_{2}$ and have valence 2 in $B(n)$. The type of $w$ is $\tau_{2}$ if $w \sim_{G} v_{1}, w$ has valence 2 in $B(n), w_{1}$ and $w_{2}$ are $G$-equivalent to $v_{2}$, and $w_{1}$ or $w_{2}$ has valence 2 in $B(n)$. If $w \sim_{G} v_{3}$, then $w$ has type $\tau_{3}$ if it has valence 2 in $B(n)$ and type $\tau_{4}$ if it has valence 3 in $B(n)$. If $w \sim_{G} v_{2}$, then $w$ has type $\tau_{5}$ if it has valence 1 in $B(n)$ and $w_{1}$ or $w_{2}$ is $G$-equivalent to $v_{1}$ and has valence 2 in $B(n)$. The type of $w$ is $\tau_{6}$ if $w \sim_{G} v_{2}, w$ has valence 2 in $B(n)$, and $w_{1}$ and $w_{2}$ are $G$-equivalent to $v_{3}$. If $w \sim_{G} v_{2}, w$ has valence 2 in $B(n)$, and $w_{1}$ and $w_{2}$ are $G$-equivalent to $v_{1}$, then $w$ has type $\tau_{7}$ if $w_{1}$ and $w_{2}$ have valence 1 in $B(n)$ and type $\tau_{8}$ if 1 of $w_{1}$ and $w_{2}$ has valence 1 in $B(n)$ and the other has valence 2 in $B(n)$. One can show inductively that each $B(n)$ is a ball, and that there is a linear recursion with the above types for which the weight vector $U=\left[\begin{array}{llllllll}0 & 1 & 1 & 2 & 0 & 1 & 1 & 1\end{array}\right]$ and the recursion matrix and 
initial vector are

$$
A=\left[\begin{array}{cccccccc}
0 & 0 & q-4 & q-5 & 0 & 0 & 0 & 0 \\
0 & 0 & 0 & 0 & 1 & 1 & 0 & 0 \\
0 & 0 & 0 & 0 & 0 & 0 & 1 & 0 \\
0 & 0 & 0 & 0 & 1 / 2 & 0 & 0 & 1 / 2 \\
0 & 0 & 0 & 0 & 1 & 0 & 0 & 0 \\
1 & 0 & 0 & 0 & 0 & 0 & 0 & 0 \\
0 & 0 & q-5 & q-6 & 0 & 0 & 0 & 0 \\
0 & 0 & 2 & 2 & 0 & 0 & 0 & 0
\end{array}\right] \quad \text { and } \quad V=\left[\begin{array}{c}
q-3 \\
2 \\
2 \\
0 \\
2 \\
1 \\
q-4 \\
2
\end{array}\right]
$$

The growth function

$$
f(z)=\frac{z^{4}+(q+3) z^{3}+(3 q-2) z^{2}+(q+3) z+1}{z^{4}+(4-q) z^{2}+1} .
$$

Now suppose that $D$ has four sides, that $v_{1}$ is fixed by an element of order three, and that $v_{2}, v_{3}$, and $v_{4}$ are in the same $G$-orbit. Let $e_{1}, e_{2}, e_{3}$, and $e_{4}$ be the edges of $D$ with boundaries $\left\{v_{1}, v_{2}\right\},\left\{v_{2}, v_{3}\right\},\left\{v_{3}, v_{4}\right\}$ and $\left\{v_{4}, v_{1}\right\}$, (respectively). Up to symmetry, there are three cases:

(i) there is an orientation-reversing element of $G$ taking $e_{2}$ to $e_{3}$;

(ii) $e_{2}$ and $e_{3}$ are each fixed setwise by rotations of order 2 in $G$; and

(iii) $e_{2}$ is fixed by a reflection in $G$ and $e_{3}$ is fixed by a rotation of order 2 in $G$.

In each case, $X=\mathbb{H}^{2}$ and the angle measure of $v_{2}$ in $D$ is $2 \pi / 3 p$, where $p$ is at least 2 .

Lemma 5.2. Let $D$ be a four-sided fundamental domain for the action of $G$ as described above. The following conditions hold for every nonnegative integer $n$.

(i) $B(n)$ is homeomorphic to a ball.

(ii) If $g \in G$ with $|g|=n$, then $g D \cap B(n-1)$ is connected.

(iii) If $g, h \in G$ with $|g|=|h|=n, g D \cap h D \neq \varnothing$, and $g D \cap h D \cap$ $B(n-1)=\varnothing$, then there is an element $g^{\prime} \in G$ with $\left|g^{\prime}\right|=n$ and $\left(g D \cup h D \cup g^{\prime} D\right) \cap B(n)$ connected.

(iv) $A$ vertex $v \in \partial B(n)$ has valence 1 or 2 , or 3 in $B(n)$. A vertex $v \in \partial B(n)$ that is $G$-equivalent to $v_{1}$ has valence 2 in $B(n)$ and is not adjacent to a vertex of valence 3 in $B(n)$.

Proof. The case $n=0$ is clear, so assume that $n \geq 0$ and that (i)-(iv) hold for $n$. Let $g \in G$ with $|g|=n+1$. We prove by contradiction that $g D \cap B(n)$ is connected. Suppose not. Then there is a polygon $C$ such that $\operatorname{int}(C)$ is a component of $X-(g D \cup B(n))$. Let $s$ be the number of edges of $\partial C$ which are in $g D$ and let $t$ be the number of edges of $\partial C$ which are in $B(n) . \partial C$ has $s+t$ vertices. The two vertices of $\partial C$ in $g D \cap B(n)$ each have angle measure at least $2 \pi / 3 p$. The $s-1$ vertices in $g D-B(n)$ each have angle measure at least $4 \pi / 3$. By (iv) the sum of the angle measures of the $t-1$ vertices of $C$ in $B(n)-g D$ is at least $(t-1) \pi-\pi / 3$. Then

$$
\frac{4 \pi}{3}-\frac{2 \pi}{p}=\operatorname{area}(D) \leq \operatorname{area}(C) \leq(s+t-2) \pi-\frac{4 \pi}{3 p}-\frac{(s-1) 4 \pi}{3}-(t-1) \pi+\frac{\pi}{3} .
$$

This is a contradiction, so $g D \cap B(n)$ is connected and (ii) holds for $n+1$. 
Now suppose that $g, h \in G$ with $|g|=|h|=n+1, g \neq h, g D \cap h D \neq$ $\varnothing$, and $g D \cap h D \cap B(n)=\varnothing$. Suppose that $g D \cap h D$ does not contain a vertex of $\partial B(n)$ and that there is no element $g^{\prime} \in G$ with $\left|g^{\prime}\right|=n+1$ and $\left(g D \cup h D \cup g^{\prime} D\right) \cap B(n)$ connected. Then there is a polygon $C$ such that $\operatorname{int}(C)$ is a component of $X-(g D \cup h D \cup B(n))$ and $\operatorname{area}(C) \geq 2 \cdot \operatorname{area}(D)$. Let $s_{1}$ be the number of edges of $C$ in $g D$, let $s_{2}$ be the number of edges of $C$ in $h D$, and let $t$ be the number of edges of $C$ in $B(n)$. Then

$$
\begin{aligned}
2\left(\frac{4 \pi}{3}-\frac{2 \pi}{p}\right) \leq & \operatorname{area}(C) \leq\left(s_{1}+s_{2}+t-2\right) \pi \\
& -\frac{\left(s_{1}-1\right) 4 \pi}{3}-\frac{\left(s_{2}-1\right) 4 \pi}{3}-(t-1) \pi+\frac{\pi}{3}-\frac{2 \pi}{p} .
\end{aligned}
$$

This simplifies to $2 \pi / 3 \leq 2 \pi / p-\left(s_{1}+s_{2}\right) \pi / 3$, a contradiction, so (iii) holds for $n+1$. It now follows as in the proof of Lemma 5.1 that $B(n+1)$ is homeomorphic to a ball.

It follows from (iii) that a vertex $w \in \partial B(n+1)$ has valence at most 3 in $B(n+1)$. If $w$ has valence 3 in $B(n+1)$, then there are elements $g$, $g^{\prime}$, and $h \in G$ such that $|g|=\left|g^{\prime}\right|=|h|=n+1, w \in\left(g D \cap g^{\prime} D \cap h D\right)$, and $g^{\prime} D \cap B(n-1)$ contains a vertex of angle measure $4 \pi / 3$ in $B(n)$. Then $g D \cap B(n)$ and $h D \cap B(n)$ are each a single vertex, so that each of the two vertices adjacent to $w$ in $\partial B(n+1)$ has valence 1 in $B(n+1)$. Thus (iv) holds for $n+1$, completing the induction step.

Using Lemma 5.2, it is easy to compute linear recursions for the above three cases. In cases (i) and (ii), the linear recursion has seven types. Let $v$ be a vertex of $\partial B(n)$ with $n \geq 1$. If $v \sim_{G} v_{1}$, then $v$ has type $\tau_{1}\left(\tau_{2}\right)$ if it has valence $1(2)$ in $B(n)$. If $v \sim_{G} v_{2}$, then $v$ has type $\tau_{3}$ if it has valence 1 in $B(n)$ and one of the adjacent vertices to $v$ in $\partial B(n)$ has type $\tau_{2}$. If $v \sim_{G} v_{2}$ and $v$ has valence 2 in $B(n)$, then the type of $v$ is $\tau_{4}$ if the two adjacent vertices to $v$ in $\partial B(n)$ are $G$-equivalent to $v_{2}, \tau_{5}$ if one of the adjacent vertices had type $\tau_{1}$, and is $\tau_{6}$ if one of the adjacent vertices has type $\tau_{2}$. The type of $v$ is $\tau_{7}$ if $v \sim_{G} v_{2}, v$ has valence 3 in $B(n)$, and the two adjacent vertices to $v$ in $\partial B(n)$ have type $\tau_{1}$. The weight vector $U=\left[\begin{array}{lllllll}0 & 1 & 0 & 1 & 1 & 1 & 2\end{array}\right]$, and the recursion matrix and initial vector are

$$
A=\left[\begin{array}{ccccccc}
0 & 0 & p-1 & p-2 & p-1 & p-1 & p-1 \\
0 & 0 & p-1 & p-1 & p-1 & p-1 & p-2 \\
0 & 0 & 2 p-2 & 2 p-2 & 2 p-3 & 2 p-3 & 2 p-4 \\
1 & 0 & 0 & 0 & 0 & 0 & 0 \\
0 & 0 & 2 p-3 & 2 p-4 & 2 p-2 & 2 p-3 & 2 p-2 \\
0 & 0 & 1 & 2 & 0 & 0 & 0 \\
0 & 0 & 1 / 2 & 0 & 0 & 1 / 2 & 0
\end{array}\right], \quad V=\left[\begin{array}{c}
3 p-3 \\
3 p-2 \\
6 p-6 \\
1 \\
6 p-6 \\
2 \\
0
\end{array}\right] .
$$

The growth function

$$
f(z)=\frac{z^{4}+(5 p-1) z^{3}+2 p z^{2}+(5 p-1) z+1}{z^{4}+(4-4 p) z^{3}+2 z^{2}+(4-4 p) z+1} .
$$

We next do case (iii), where $e_{2}$ is fixed by a reflection and $e_{3}$ is invariant under a rotation of order 2 . While the growth function is the same as for the first two cases, the combinatorics is more complicated because of the reflection. We 
need to be careful of orientations, and we assume without loss of generailty that $v_{1}, v_{2}, v_{3}$, and $v_{4}$ are in counterclockwise order. Given a vertex $v \in \partial B(n)$ with $n \geq 1$, let $l v(v)(r v(v))$ be the adjacent vertex to $v$ in $\partial B(n)$ that is counterclockwise (clockwise) to $v$, and let $l e(v)(r e(v))$ be the adjacent edge to $v$ in $\partial B(n)$ that is counterclockwise (clockwise) to $v$. (So $\partial l e(v)=$ $l v(v) \cup v$ and $\partial r e(v)=r v(v) \cup v$.) If $v$ is $G$-equivalent to $v_{1}$, then $v$ has type $\tau_{1}\left(\tau_{2}\right)$ if $v$ has valence 1 (two) in $B(n)$. The type of $v$ is $\tau_{3}\left(\tau_{4}\right)$ if $v \sim_{G} v_{2}, v$ has valence 1 in $B(n), r v(v)$ has type $\tau_{2}$, and $l e(v)$ is $G$ equivalent to $e_{3}\left(e_{2}\right)$. If $v \sim_{G} v_{2}$ and $v$ has valence 2 in $B(n)$, then the type of $v$ is $\tau_{i}$ for some $i$ with $5 \leq i \leq 10$. The type is $\tau_{5}$ if $l e(v)$ is $G$ equivalent to $e_{3}$ and $r e(v)$ is $G$-equivalent to $e_{2}, \tau_{6}$ if $l v(v)$ and $r v(v)$ each have type $\tau_{1}, \tau_{7}$ if $l v(v)$ has type $\tau_{1}$ and $r v(v)$ has type $\tau_{2}, \tau_{8}$ if $l e(v)$ is $G$-equivalent to $e_{2}$ and $r v(v)$ has type $\tau_{1}, \tau_{9}$ if $l e(v)$ is $G$-equivalent to $e_{2}$ and $r v(v)$ has type $\tau_{2}$, and $\tau_{10}$ if $l e(v)$ and $r e(v)$ are $G$-equivalent to $e_{3}$. Finally, $v$ has type $\tau_{11}$ if $v \sim_{G} v_{2}, v$ has valence 3 in $B(n)$, $l e(v)$ is $G$-equivalent to $e_{3}$, and $r v(v)$ has type $\tau_{1}$. Let $p=2 q$. The weight vector $U=\left[\begin{array}{lllllllllll}0 & 1 & 0 & 0 & 1 & 1 & 1 & 1 & 1 & 1 & 2\end{array}\right]$, the recursion matrix

$$
A=\left[\begin{array}{ccccccccccc}
0 & 0 & p-1 & p & p-1 & p & p & p-2 & p-2 & p-2 & p-2 \\
0 & 0 & p-1 & p-1 & p-1 & p-2 & p-2 & p-1 & p-1 & p & p-1 \\
0 & 0 & p-1 & p-2 & p-2 & p-2 & p-2 & p-1 & p-1 & p & p-1 \\
0 & 0 & p-1 & p-1 & p-1 & p-2 & p-2 & p-1 & p-1 & p-2 & p-2 \\
1 & 0 & 0 & 0 & 0 & 0 & 0 & 0 & 0 & 0 & 0 \\
0 & 0 & q-1 & q & q-1 & q & q & q-1 & q-1 & q-1 & q-1 \\
0 & 0 & 1 & 0 & 1 & 0 & 0 & 0 & 0 & 0 & 0 \\
0 & 0 & p-1 & p-1 & p-1 & p & p-1 & p-2 & p-2 & p-2 & p-2 \\
0 & 0 & 0 & 0 & 0 & 0 & 0 & 1 & 1 & 0 & 0 \\
0 & 0 & q-1 & q-1 & q-1 & q-1 & q-1 & q & q-1 & q & q \\
0 & 0 & 1 / 2 & 1 / 2 & 0 & 0 & 1 / 2 & 0 & 1 / 2 & 0 & 0
\end{array}\right]
$$

and the transpose of the initial vector is

$$
\left[\begin{array}{ccccccccccc}
6 q-3 & 6 q-2 & 6 q-3 & 6 q-3 & 1 & 3 q-2 & 1 & 6 q-3 & 1 & 3 q-1 & 0
\end{array}\right] \text {. }
$$

The growth function

$$
f(z)=\frac{z^{4}+(5 p-1) z^{3}+2 p z^{2}+(5 p-1) z+1}{z^{4}+(4-4 p) z^{3}+2 z^{2}+(4-4 p) z+1} .
$$

\section{THE EXCEPTIONAL PENTANGUlaR DOMAINS}

To complete the analysis, it remains to consider the cases where $D$ has five sides and there are three vertices of $D$ that are not adjacent and have valence 3. We will see that these are exactly the cases for which $D$ has five sides and there are buried domains, and that the growth functions are not reciprocal. Let $v_{1}, v_{2}, v_{3}, v_{4}$, and $v_{5}$ be the vertices of $D$ (in clockwise or counterclockwise order), and assume that $v_{1}, v_{2}$, and $v_{4}$ each have valence 3 . Then $v_{3}$ is fixed by a generator of order $q$ and $v_{5}$ is fixed by a generator of order $r$, and $1 / q+1 / r \leq 1 / 2$. As shown in Figure $1, G$ is the triangle group $\Delta(2, q, r)$. We will analyze the growth function in two cases, as follows:

(i) $q \geq 4$ and $r \geq 4$; and

(ii) $q=3$ and $r \geq 6$.

These two cases are the most complicated of the exceptional cases. Let $n$ be a positive integer such that $B(n)$ is homeomorphic to a ball, and let $v$ be 
a vertex of $\partial B(n)$. Let $w_{1}$ and $w_{2}$ be the adjacent vertices to $v$ in $\partial B(n)$, and let $x_{1}$ and $x_{2}$ be vertices of $\partial B(n)$ such that $x_{1}$ and $v$ are adjacent to $w_{1}$ and $x_{2}$ and $v$ are adjacent to $w_{2}$. Then the combinatorics of the vertices in $\partial B(n+1)$ that are in domains $g D$ that also contain $v$ depend not only on the equivalence classes and valences in $B(n)$ of $v, w_{1}$, and $w_{2}$, but also on whether $w_{1}$ and $x_{1}$ (or $w_{2}$ and $x_{2}$ ) both have valence 2 in $B(n)$ and valence 3 in the tessellation. Since one can show inductively that there are never three adjacent vertices in $\partial B(m)$, for some positive integer $m$, that have valence 2 in $B(m)$ and valence 3 in the tessellation, we can restrict the number of types (and hence the size of the recursion matrix) by changing the convention in how we compute the columns of the recursion matrix. The type of $v$ will depend only on $v, w_{1}$, and $w_{2}$, and not on $x_{1}$ or $x_{2}$. However, in computing the column of the recursion matrix corresponding to a vertex of type $\tau_{i}$, we will choose a representative vertex $w$ in $B(m)$ for which it is not true that there are vertices $w_{1}$ and $x_{1}$ which both have valence 2 in $B(m)$ and valence 3 in the tessellation and such that $x_{1}$ and $w$ are adjacent to $w_{1}$ in $\partial B(m)$. If $w$ and an adjacent vertex $w_{1}$ both have valence 2 in $B(m)$ and valence 3 in the tessellation, then the $i$ th column of $A$ will have some negative entries to correct for the mistakes in computing the output of the other vertex $x_{1}$ that is adjacent to $w_{1}$.

We first do case (i), where $q \geq 4$ and $r \geq 4$ (see Figure 7). Let $v$ be a vertex of $\partial B(n)$ with $n$ a positive integer such that $B(n)$ is homeomorphic to a ball, and let $w_{1}$ and $w_{2}$ be the adjacent vertices to $v$ in $\partial B(n)$ such that $w_{1}, v$, and $w_{2}$ are in the same cyclic order as $v_{1}, v_{2}, v_{3}, v_{4}$, and $v_{5}$. First suppose that $v \sim_{G} v_{1}$ and $v$ has valence 1 in $B(n)$. Then $v$ has type $\tau_{1}$ if $w_{1} \sim_{G} v_{3}$ and $w_{2} \sim_{G} v_{5}$, type $\tau_{2}$ if $w_{1} \sim_{G} v_{5}, w_{2} \sim_{G} v_{1}$, and $w_{2}$ has valence 2 in $B(n)$, and týpe $\tau_{3}$ if $w_{1} \sim_{G} v_{1}, w_{2} \sim_{G} v_{3}$, and $w_{1}$ has valence 2 in $B(n)$. Now suppose that $v \sim_{G} v_{1}$ and $v$ has valence 2 in $B(n)$. The type of $v$ is $\tau_{4}\left(\tau_{5}\right)$ if $w_{1} \sim_{G} v_{1}, w_{2} \sim_{G} v_{5}$, and $w_{1}$ has valence 1 (2) in $B(n)$.

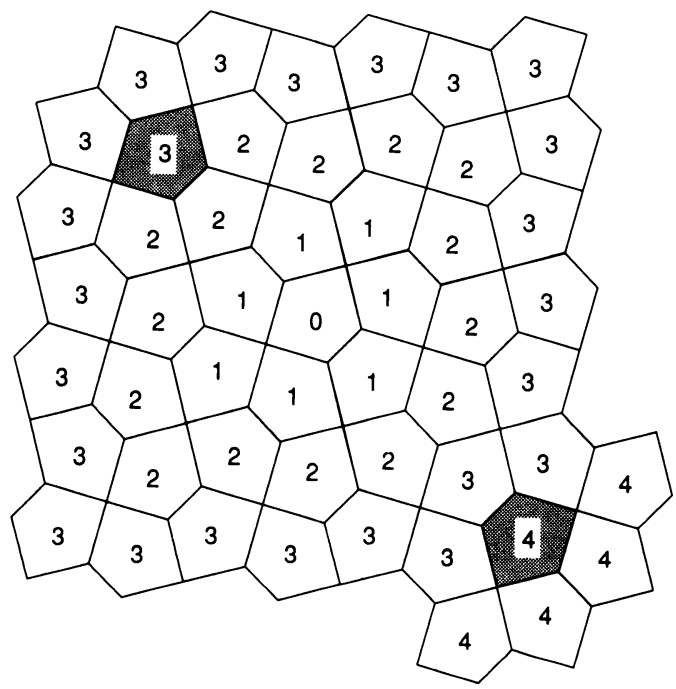

FIGURE 7. $\Delta(2,4,4)$ 
If $w_{1} \sim_{G} v_{3}$ and $w_{2} \sim_{G} v_{1}$, then $v$ has type $\tau_{6}$ if $w_{2}$ has valence 1 in $B(n)$ and type $\tau_{7}$ if $w_{2}$ has valence 2 in $B(n)$. The type of $v$ is $\tau_{8}$ if $w_{1} \sim_{G} v_{5}$ and $w_{2} \sim_{G} v_{3}$. Next suppose that $v \sim_{G} v_{5}$ and $v$ has valence 1 in $B(n)$. Then $w_{1} \sim_{G} v_{1}$ and $w_{2} \sim_{G} v_{1}$. The type of $v$ is $\tau_{9}$ if $w_{1}$ has valence 2 in $B(n)$ and $w_{2}$ has valence 1 in $B(n), \tau_{10}$ if $w_{1}$ has valence 1 in $B(n)$ and $w_{2}$ has valence 2 in $B(n)$, and $\tau_{11}$ if $w_{1}$ has valence 2 in $B(n)$ and $w_{2}$ has valence 2 in $B(n)$. Next suppose that $v \sim_{G} v_{3}$ and $v$ has valence 1 in $B(n)$. Then $w_{1} \sim_{G} v_{1}$ and $w_{2} \sim_{G} v_{1}$. The type of $v$ is $\tau_{12}$ if $w_{1}$ has valence 2 in $B(n)$ and $w_{2}$ has valence 1 in $B(n), \tau_{13}$ if $w_{1}$ has valence 1 in $B(n)$ and $w_{2}$ has valence 2 in $B(n)$, and $\tau_{14}$ if $w_{1}$ has valence 2 in $B(n)$ and $w_{2}$ has valence 2 in $B(n)$. Next suppose that $v \sim_{G} v_{5}$ and $v$ has valence 2 in $B(n)$. Then $w_{1} \sim_{G} v_{1}$ and $w_{2} \sim_{G} v_{1}$. The type of $v$ is $\tau_{15}$ if $w_{1}$ has valence 1 in $B(n)$ and $w_{2}$ has valence 1 in $B(n), \tau_{16}$ if $w_{1}$ has valence 1 in $B(n)$ and $w_{2}$ has valence 2 in $B(n)$, and $\tau_{17}$ if $w_{1}$ has valence 2 in $B(n)$ and $w_{2}$ has valence 1 in $B(n)$. Next suppose that $v \sim_{G} v_{3}$ and $v$ has valence 2 in $B(n)$. Then $w_{1} \sim_{G} v_{1}$ and $w_{2} \sim_{G} v_{1}$. The type of $v$ is $\tau_{18}$ if $w_{1}$ has valence 1 in $B(n)$ and $w_{2}$ has valence 1 in $B(n), \tau_{19}$ if $w_{1}$ has valence 1 in $B(n)$ and $w_{2}$ has valence 2 in $B(n)$, and $\tau_{20}$ if $w_{1}$ has valence 2 in $B(n)$ and $w_{2}$ has valence 1 in $B(n)$. Once can show inductively that each combinatorial ball is homeomorphic to a ball and that there is a linear recursion on the vertices of the boundaries of the combinatiorial balls with the above types. There is one final type, $\tau_{21}$, that corresponds to a buried domain and not to a vertex. The weight vector $U$ is

\section{$\left[\begin{array}{lllllllllllllllllllll}0 & 0 & 0 & 1 & 1 & 1 & 1 & 1 & 0 & 0 & 0 & 0 & 0 & 0 & 1 & 1 & 1 & 1 & 1 & 1 & 1\end{array}\right]$,}

the transpose of the initial vector $V$ is

$\left[\begin{array}{lllllllllllllllllllll}1 & q-2 & r-2 & q-2 & 0 & r-2 & 0 & 1 & q-3 & 0 & 1 & 0 & r-3 & 1 & 1 & 0 & 0 & 1 & 0 & 0 & 0\end{array}\right]$,

and the recursion matrix $A$ is

$\left[\begin{array}{cccccccccccccccccccccc}0 & 0 & 0 & 0 & 0 & 0 & 0 & 0 & 0 & 0 & 0 & 0 & 0 & 0 & 0 & 0 & 0 & 0 & 0 & 0 & 0 \\ 0 & 0 & 1 / 2 & 0 & 0 & 0 & -1 & 0 & 0 & 0 & 0 & q-3 & q-5 / 2 & q-3 & 0 & 0 & 0 & q-7 / 2 & q-7 / 2 & q-4 & 0 \\ 0 & 1 / 2 & 0 & 0 & -1 & 0 & 0 & 0 & r-5 / 2 & r-3 & r-3 & 0 & 0 & 0 & r-7 / 2 & r-4 & r-7 / 2 & 0 & 0 & 0 & 0 \\ 0 & 0 & 0 & 0 & 0 & 0 & -1 & 0 & 0 & 0 & 0 & q-3 & q-2 & q-3 & 0 & 0 & 0 & q-3 & q-3 & q-4 & 0 \\ 0 & 0 & 0 & 0 & 0 & 0 & 0 & 0 & 0 & 0 & 0 & 1 & 0 & 1 & 0 & 0 & 0 & 0 & 0 & 1 & 0 \\ 0 & 0 & 0 & 0 & -1 & 0 & 0 & 0 & r-2 & r-3 & r-3 & 0 & 0 & 0 & r-3 & r-4 & r-3 & 0 & 0 & 0 & 0 \\ 0 & 0 & 0 & 0 & 0 & 0 & 0 & 0 & 0 & 1 & 1 & 0 & 0 & 0 & 0 & 1 & 0 & 0 & 0 & 0 & 0 \\ 1 & 0 & 0 & 0 & 1 / 2 & 0 & 1 / 2 & 0 & 0 & 0 & 0 & 0 & 0 & 0 & 0 & 0 & 0 & 0 & 0 & 0 & 0 \\ 0 & 0 & 0 & 0 & 0 & 0 & -1 & 0 & 0 & 0 & 0 & q-3 & q-3 & q-3 & 0 & 0 & 0 & q-4 & q-4 & q-4 & 0 \\ 0 & 0 & 0 & 0 & 0 & 0 & 0 & 0 & 0 & 0 & 0 & 0 & 0 & 0 & 0 & 0 & 0 & 0 & 0 & 0 & 0 \\ 1 / 2 & 0 & 0 & 0 & 0 & 0 & 1 & 0 & 0 & 0 & 0 & 1 / 2 & 0 & 0 & 0 & 0 & 0 & 1 / 2 & 0 & 1 / 2 & 0 \\ 0 & 0 & 0 & 0 & 0 & 0 & 0 & 0 & 0 & 0 & 0 & 0 & 0 & 0 & 0 & 0 & 0 & 0 & 0 & 0 & 0 \\ 0 & 0 & 0 & 0 & -1 & 0 & 0 & 0 & r-3 & r-3 & r-3 & 0 & 0 & 0 & r-4 & r-4 & r-4 & 0 & 0 & 0 & 0 \\ 1 / 2 & 0 & 0 & 0 & 1 & 0 & 0 & 0 & 0 & 1 / 2 & 0 & 0 & 0 & 0 & 1 / 2 & 1 / 2 & 0 & 0 & 0 & 0 & 0 \\ 0 & 0 & 0 & 0 & 0 & 0 & 0 & 0 & 0 & 0 & 0 & 0 & 0 & 0 & 0 & 0 & 0 & 0 & 0 & 0 & 0 \\ 0 & 0 & 0 & 0 & 0 & 0 & 0 & 0 & 0 & 0 & 0 & 0 & 0 & 0 & 0 & 0 & 0 & 0 & 0 & 0 & 0 \\ 0 & 0 & 1 & 0 & 0 & 0 & 0 & 0 & 0 & 0 & 0 & 0 & 0 & 0 & 0 & 0 & 0 & 0 & 0 & 0 & 0 \\ 0 & 0 & 0 & 0 & 0 & 0 & 0 & 0 & 0 & 0 & 0 & 0 & 0 & 0 & 0 & 0 & 0 & 0 & 0 & 0 & 0 \\ 0 & 1 & 0 & 0 & 0 & 0 & 0 & 0 & 0 & 0 & 0 & 0 & 0 & 0 & 0 & 0 & 0 & 0 & 0 & 0 & 0 \\ 0 & 0 & 0 & 0 & 0 & 0 & 0 & 0 & 0 & 0 & 0 & 0 & 0 & 0 & 0 & 0 & 0 & 0 & 0 & 0 & 0 \\ 0 & 0 & 0 & 0 & 1 / 2 & 0 & 1 / 2 & 0 & 0 & 0 & 0 & 0 & 0 & 0 & 0 & 0 & 0 & 0 & 0 & 0 & 0\end{array}\right]$.




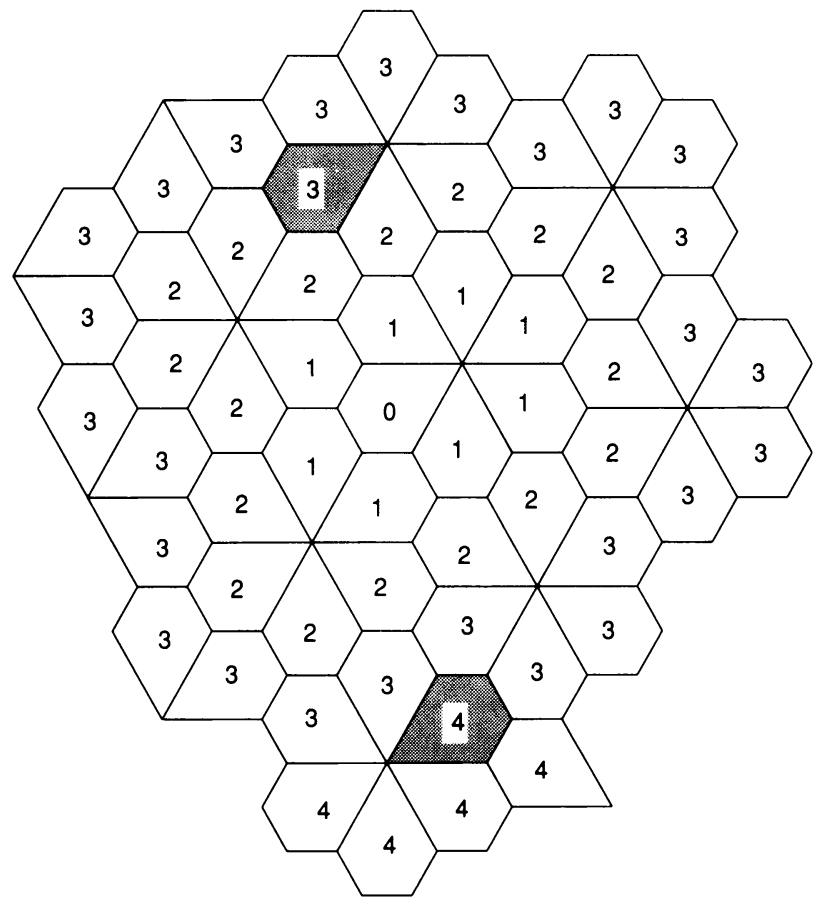

FiguRE 8. $\Delta(2,3,6)$

The growth function

$$
f(z)=\frac{-z^{6}-z^{5}+(q r-2 r-2 q+3) z^{4}+(q r-q-r+2) z^{3}+(q r-3) z^{2}+(q+r-1) z+1}{z^{4}+(q r-2 q-2 r+2) z^{2}+1} .
$$

We now do case (ii), where $q=3$ and $r \geq 6$ (see Figure 8). Let $v$ be a vertex of $\partial B(n)$ with $n$ a positive integer such that $B(n)$ is homeomorphic to a ball, and let $w_{1}$ and $w_{2}$ be the adjacent vertices to $v$ in $\partial B(n)$ such that $w_{1}, v$, and $w_{2}$ are in the same cyclic order as $v_{1}, v_{2}, v_{3}, v_{4}$, and $v_{5}$. First suppose that $v \sim_{G} v_{1}$ and $v$ has valence 1 in $B(n)$. Then $v$ has type $\tau_{1}$ if $w_{1} \sim_{G} v_{3}, w_{2} \sim_{G} v_{5}$, and $w_{1}$ has valence 2 in $B(n)$, type $\tau_{2}$ if $w_{1} \sim_{G} v_{5}$, $w_{2} \sim_{G} v_{1}$, and $w_{2}$ has valence 2 in $B(n)$, type $\tau_{3}$ if $w_{1} \sim_{G} v_{1}, w_{2} \sim_{G} v_{3}$, $w_{1}$ has valence 2 in $B(n)$, and $w_{2}$ has valence 1 in $B(n)$, and type $\tau_{4}$ if $w_{1} \sim_{G} v_{1}, w_{2} \sim_{G} v_{3}$, and $w_{1}$ and $w_{2}$ have valence 2 in $B(n)$. Now suppose that $v \sim_{G} v_{1}$ and $v$ has valence 2 in $B(n)$. The type of $v$ is $\tau_{5}\left(\tau_{6}\right)$ if $w_{1} \sim_{G} v_{1}, w_{2} \sim_{G} v_{5}$, and $w_{1}$ has valence $1(2)$ in $B(n)$. If $w_{1} \sim_{G} v_{3}$ and $w_{2} \sim_{G} v_{1}$, then $v$ has type $\tau_{7}$ if $w_{1}$ and $w_{2}$ have valence 1 in $B(n)$, type $\tau_{8}$ if $w_{1}$ has valence 2 in $B(n)$ and $w_{2}$ has valence 1 in $B(n)$, and type $\tau_{9}$ if $w_{1}$ has valence 1 in $B(n)$ and $w_{2}$ has valence 2 in $B(n)$. The type of $v$ is $\tau_{10}$ if $w_{1} \sim_{G} v_{5}, w_{2} \sim_{G} v_{3}$, and $w_{2}$ has valence 1 in $B(n)$. If $v \sim_{G} v_{5}$ and $v$ has valence 1 in $B(n)$, the $v$ has type $\tau_{11}$ if $w_{1} \sim_{G} v_{1}, w_{2} \sim_{G} v_{1}$, and $w_{1}$ and $w_{2}$ have valence 2 in $B(n)$. Next suppose that $v \sim_{G} v_{3}$ and $v$ has valence 1 in $B(n)$. Then $w_{1} \sim_{G} v_{1}$ and $w_{2} \sim_{G} v_{1}$. The type of $v$ is $\tau_{12}$ if $w_{1}$ has valence 1 in $B(n)$ and $w_{2}$ has valence 2 in $B(n)$, and $\tau_{13}$ if $w_{1}$ and $w_{2}$ has valence 2 in $B(n)$. Next suppose that $v \sim_{G} v_{5}$ and $v$ has valence 2 in $B(n)$. Then $w_{1} \sim_{G} v_{1}$ and $w_{2} \sim_{G} v_{1}$. The type of $v$ is $\tau_{14}$ if $w_{1}$ and $w_{2}$ have valence 1 in $B(n), \tau_{15}$ if $w_{1}$ has valence 2 in $B(n)$ and $w_{2}$ has valence 1 in $B(n)$, and $\tau_{16}$ if $w_{1}$ and $w_{2}$ have valence 2 in $B(n)$. Next suppose that 
$v \sim_{G} v_{3}$ and $v$ has valence 2 in $B(n)$. Then $w_{1} \sim_{G} v_{1}$ and $w_{2} \sim_{G} v_{1}$. The type of $v$ is $\tau_{17}$ if $w_{1}$ and $w_{2}$ have valence 1 in $B(n)$, and $\tau_{18}$ if $w_{1}$ has valence 1 in $B(n)$ and $w_{2}$ has valence 2 in $B(n)$. The type of $v$ is $\tau_{19}$ if $v \sim_{G} v_{5}, v$ has valence three in $B(n), w_{1} \sim_{G} v_{1}, w_{2} \sim_{G} v_{1}, w_{1}$ has valence 2 in $B(n)$, and $w_{2}$ has valence 1 in $B(n)$. Once can show inductively that each combinatorial ball is homeomoprhic to a ball and that there is a linear recursion on the vertices of the boundaries of the combinatiorial balls with the above types. There is one final type, $\tau_{20}$, that corresponds to a buried domain and not to a vertex. The weight vector $U$ is

$$
\left[\begin{array}{llllllllllllllllllll}
0 & 0 & 0 & 0 & 1 & 1 & 1 & 1 & 1 & 1 & 0 & 0 & 0 & 1 & 1 & 1 & 1 & 1 & 2 & 1
\end{array}\right] \text {, }
$$

the transpose of the initial vector $V$ is

$$
\left[\begin{array}{lllllllllllllllllllll}
1 & 1 & r-3 & 1 & 1 & 0 & r-2 & 0 & 0 & 1 & 1 & r-3 & 1 & 1 & 0 & 0 & 1 & 0 & 0 & 0
\end{array}\right] \text {, }
$$

and the recursion matrix $A$ is

$$
\left[\begin{array}{cccccccccccccccccccc}
0 & 0 & 0 & 0 & 0 & 0 & 0 & 0 & 0 & 0 & 0 & 0 & 0 & 0 & 0 & 0 & 0 & 0 & 0 & 0 \\
0 & 0 & 1 / 2 & 0 & 0 & 0 & 0 & 0 & -1 & 0 & 0 & 1 / 2 & 0 & 0 & 0 & 0 & 0 & 0 & 0 & 0 \\
0 & 0 & 0 & 0 & 0 & -1 & 0 & 0 & 0 & 0 & r-3 & 0 & 0 & r-4 & r-4 & r-4 & 0 & 0 & r-5 & 0 \\
0 & 1 / 2 & 0 & 0 & 0 & 0 & 0 & 0 & 0 & 0 & 0 & 0 & 0 & 1 / 2 & 1 / 2 & 0 & 0 & 0 & 1 / 2 & 0 \\
0 & 0 & 0 & 0 & 0 & 0 & 0 & 0 & -1 & 0 & 0 & 1 & 0 & 0 & 0 & 0 & 0 & 0 & 0 & 0 \\
0 & 0 & 0 & 0 & 0 & 0 & 0 & 0 & 0 & 0 & 0 & 0 & 1 & 0 & 0 & 0 & 0 & 0 & 0 & 0 \\
0 & 0 & 0 & 0 & 0 & 0 & 0 & 0 & 0 & 0 & r-4 & 0 & 0 & r-3 & r-4 & r-5 & 0 & 0 & r-5 & 0 \\
0 & 0 & 0 & 0 & 0 & -1 & 0 & 0 & 0 & 0 & 1 & 0 & 0 & 0 & 1 & 1 & 0 & 0 & 1 & 0 \\
0 & 0 & 0 & 0 & 0 & 0 & 0 & 0 & 0 & 0 & 1 & 0 & 0 & 0 & 0 & 1 & 0 & 0 & 0 & 0 \\
1 & 0 & 0 & 0 & 0 & 0 & 0 & 0 & 1 & 0 & 0 & 0 & 0 & 0 & 0 & 0 & 0 & 0 & 0 & 0 \\
0 & 0 & 0 & 0 & 0 & 0 & 0 & 0 & 0 & 0 & 0 & 0 & 0 & 0 & 0 & 0 & 0 & 0 & 0 & 0 \\
0 & 0 & 0 & 0 & 0 & -1 & 0 & 0 & 0 & 0 & r-3 & 0 & 0 & r-4 & r-4 & r-4 & 0 & 0 & r-5 & 0 \\
1 / 2 & 0 & 0 & 0 & 0 & 1 & 0 & 0 & 0 & 0 & 0 & 0 & 0 & 1 / 2 & 0 & 0 & 0 & 0 & 0 & 0 \\
0 & 0 & 0 & 0 & 0 & 0 & 0 & 0 & 0 & 0 & 0 & 0 & 0 & 0 & 0 & 0 & 0 & 0 & 0 & 0 \\
0 & 0 & 1 & 0 & 0 & 0 & 0 & -1 & -1 & 0 & 0 & 0 & 0 & 0 & 0 & 0 & 0 & 0 & 0 & 0 \\
0 & 0 & 0 & 1 & 0 & 0 & 0 & 0 & 1 & 0 & 0 & 0 & 0 & 0 & 0 & 0 & 0 & -1 & 0 & 0 \\
0 & 0 & 0 & 0 & 0 & 0 & 0 & 0 & 0 & 0 & 0 & 0 & 0 & 0 & 0 & 0 & 0 & 0 & 0 & 0 \\
0 & 1 & 0 & 0 & 0 & 0 & 0 & 0 & 0 & 0 & 0 & 0 & 0 & 0 & 0 & 0 & 0 & 0 & 0 & 0 \\
0 & 0 & 0 & 0 & 0 & 0 & 0 & 1 / 2 & 0 & 0 & 0 & 0 & 0 & 0 & 0 & 0 & 0 & 1 / 2 & 0 & 0 \\
0 & 0 & 0 & 0 & 0 & 1 / 2 & 0 & 0 & 1 / 2 & 0 & 0 & 0 & 0 & 0 & 0 & 0 & 0 & 0 & 0 & 0
\end{array}\right]
$$

The growth function

$$
f(z)=\frac{-z^{6}-z^{5}+(r-3) z^{4}+(2 r-1) z^{3}+(3 r-3) z^{2}+(r+2) z+1}{z^{4}+(4-r) z^{2}+1} .
$$

\section{7. $f(1)$ AND THE EULER CHARACTERISTIC}

Let $G$ be a cocompact, discrete group of isometries of $\mathbb{H}^{2}$, let $D$ be a Dirichlet region for the action of $G$, and let $\Sigma$ be the associated full geometric generating set. In the above sections, we showed that the combinatorial ball $B(n)$ is always homeomorphic to a ball, and that there is a linear recursion on the vertices of the boundaries of the combinatorial balls. Using this, it is easy to adapt the argument from [4] to prove that $f(1)=1 / \chi(G)$ unless 1 is an eigenvalue of the recursion matrix $A$.

It is more convenient to work with the growth series

$$
h_{b}(z)=\sum_{n=0}^{\infty} b_{n} z^{n}
$$


where $b_{n}$ is the number of elements of $G$ of word norm at most $n$ in $\Sigma$. Since $s_{n}=b_{n}-b_{n-1}, h(z)=(1-z) h_{b}(z)$.

If $D$ is one of the Dirichlet regions considered in $\S \S 1-3$, then the types correspond to primitive angles and angle-pairs. In particular, if the vertices $g_{1} \in \partial B\left(n_{1}\right)$ and $g_{2} \in \partial B\left(n_{2}\right)$ have the same type, then the angle measure of $g_{1}$ in $B\left(n_{1}\right)$ is the angle measure of $g_{2}$ in $B\left(n_{2}\right)$. Let $\Theta$ be the row vector with $i$ th coordinate the angle measure of a vertex of type $\tau_{i}$, and let $S$ be the row vector with $i$ th coordinate 1 . Then for every positive integer $n, \Theta A^{n-1} V$ is the sum of the angle measures of the vertices of the boundary of $B(n)$, and $S A^{n-1} V$ is the number of edges of the boundary of $B(n)$.

If $D$ is one of the Dirichlet regions considered in $\S \S 4-6$, then we assumed (by possibly replacing $D$ by a Dirchlet region with the same full geometric generating set) that the angle measure of a vertex of $D$ was $2 \pi$ divided by its valence in the tessellation. With this assumption, if the vertices $g_{1} \in \partial B\left(n_{1}\right)$ and $g_{2} \in \partial B\left(n_{2}\right)$ have the same type, then the angle measure of $g_{1}$ in $B\left(n_{1}\right)$ is the angle measure of $g_{2}$ in $B\left(n_{2}\right)$. Let $\Theta$ be the row vector with $i$ th coordinate the angle measure of a vertex of type $\tau_{i}$ if $\tau_{i}$ is the type of a vertex and with $i$ th coordinate 0 if $\tau_{i}$ is the type of a buried domain. Let $S$ be the row vector with $i$ th coordinate 1 if $\tau_{i}$ is the type of a vertex and with $i$ th coordinate 0 if $\tau_{i}$ is the type of a buried domain. Then for every positive integer $n, \Theta A^{n-1} V$ is the sum of the angle measures of the vertices of the boundary of $B(n)$, and $S A^{n-1} V$ is the number of edges of the boundary of $B(n)$.

By the Gauss-Bonnet theorem, if $n$ is a positive integer then

$$
\operatorname{area}(B(n))=\pi\left(S A^{n-1} V-2\right)-\Theta A^{n-1} V .
$$

Since the area of $B(n)$ is $b_{n} \times$ area $(D)$, by the Gauss-Bonnet theorem

$$
\operatorname{area}(B(n))=-2 \pi \chi b_{n} \text {. }
$$

Thus

$$
b_{n}=\frac{1}{\chi}+\frac{\Theta-\pi S}{2 \pi \chi} A^{n-1} V,
$$

and

$$
\begin{aligned}
h_{b}(z) & =1+\sum_{n=1}^{\infty} b_{n} z^{n}=1+\sum_{n=1}^{\infty} \frac{1}{\chi} z^{n}+\frac{\Theta-\pi S}{2 \pi \chi} A^{n-1} V z^{n} \\
& =1+z \sum_{m=0}^{\infty} \frac{z^{m}}{\chi}+z \sum_{m=0}^{\infty} \frac{\Theta-\pi S}{2 \pi \chi} z^{m} A^{m} V .
\end{aligned}
$$

Since this is the Maclaurin series of the function

$$
1+\frac{z}{\chi(1-z)}+\frac{z}{2 \pi \chi}(\Theta-\pi S)(I-z A)^{-1} V,
$$

multiplying by $1-z$ proves the following theorem.

Theorem. Let $G$ be a cocompact, discrete group of isometries of $\mathbb{H}^{2}$, and let $\Sigma$ be the full geometric generating set for $G$ with respect to a Dirichlet region $D$. Then with the notation as above, 


$$
f(z)=(1-z)+\frac{z}{\chi}+\frac{z(1-z)}{2 \pi \chi}(\Theta-\pi S)(I-z A)^{-1} V .
$$

In particular, $f(1)=1 / \chi$ if 1 is not an eigenvalue of the recursion matrix $A$.

\section{EXAMPLES}

The analysis in $\S 2$ suggests an algorithm to compute the growth function of a planar group, with a full geometric generating set, as long as the Dirichlet domain $D$ has at least six sides. Using this algorithm, we computed all of the examples when $G$ is the fundamental group of a closed, nonorientable surface of genus 3 or of a closed, orientable surface of genus 2. Up to combinatorial equivalence, there are 65 different ways to get a closed, nonorientable surface of genus 3 from gluing sides of a fundamental polygon $D$ with no vertex of valence 1 or 2 in the quotient, and there are 122 different ways for a closed, orientable surface of genus 2 . In this section we list all of the growth functions, with full geometric generating sets, for these two groups and these fundamental polygons. Since all of the growth functions are reciprocal and have numerator and denominator of even degree, for convenience we denote the growth function by listing the coefficients of the numerator and denominator from the constant term up to the point of symmetry. For example, we denote the growth function

$$
\frac{1+3 x+4 x^{2}+3 x^{3}+x^{4}}{1-7 x-12 x^{2}-7 x^{3}+x^{4}}
$$

by $\left(\begin{array}{lll}1 & 3\end{array}\right) /(1-7-12)$. Following the growth function, we list the different sets of edge identifications which give this growth function (here we are numbering the sides of the $n$-gon $D$ by $0,1, \ldots, n-1$, and for the nonorientable group we use ${ }^{*}$ to denote when a generator reverses orientation). We also list the relators of the associated presentations, where the generators are labelled $a, b, c, \ldots$ and their inverses are labelled $A, B, C, \ldots$.

All of the growth functions are given with the numerator and denominator relatively prime. All of the denominators are irreducible. The 22 growth functions labelled * have denominators which are Salem polynominals. Each of the other 23 denominators has exactly four real zeroes and all other zeroes on the unit circle. All of the growth functions $f(z)$ have $f(1)=1 / \chi$ except for $(17) /(1-23)$.

\section{Growth functions for a nonorientable, genus 3 surface group.}

\section{Sides}

$$
\begin{array}{rll}
(110) /(1-14) * \quad & & \\
& (13,24,5 * 0) & c c b A B a \\
& (13,25,4 * 0) & c b a B c a \\
& (13,2 * 4,5 * 0) & c c b a B a \\
& (13,2 * 5,4 * 0) & c b A B c a \\
& (14,25,3 * 0) & c a B c B a \\
(1 * 2,3 * 4,5 * 0) & c c b b a a \\
& (1 * 2,3 * 0,4 * 5) & b C C b a a \\
& (1 * 3,2 * 5,4 * 0) & c b A C b a
\end{array}
$$




\section{Sides}

$(16) /(1-] 10) *$

$(13,25,46,7 * 0) \quad d d c a \quad A B C b$

$(13,26,4 * 0,57) \quad c D c a \quad A B D b$

$(13,2 * 5,46,7 * 0) \quad d d c a \quad A B c b$

$(13,2 * 6,4 * 0,57) \quad c D c a \quad A B d b$

$(14,25,3 * 7,6 * 0) \quad d c B a \quad A C d b$

$(14,27,3 * 5,6 * 0) \quad d b C a \quad A C D b$

$(14,2 * 5,3 * 0,6 * 7) \quad c a B a \quad B D D c$

$(15,2 * 6,37,4 * 0) \quad d a B a \quad B c D c$

$(1 * 2,3 * 5,4 * 0,6 * 7) \quad$ cbaa $\quad B D D c$

$(1 * 3,2 * 6,4 * 0,5 * 7) \quad c D b a \quad A C d b$

$\left(\begin{array}{lll}1 & 10 & 22\end{array}\right) /\left(\begin{array}{lll}1 & -8 & -30\end{array}\right)$

$(13,24,50,6 * 7) \quad c b A B a \quad C d d$

$(13,2 * 4,50,6 * 7) \quad c b a B a \quad C d d$

$(14,2 * 3,5 * 6,7 * 0) \quad d d c c a \quad A b b$

$(14,2 * 3,5 * 0,6 * 7) \quad c D D c a \quad A b b$

$(16-2) /(1-1210) *$

$(13,25,4 * 7,6 * 0) \quad A B C d b \quad d c a$

$(13,2 * 5,4 * 7,6 * 0) \quad A B D c b \quad d c a$

$(14,25,36,7 * 0) \quad d d c B a \quad A C b$

$(14,26,37,5 * 0) \quad d b C d a \quad A C b$

$(14,26,3 * 0,5 * 7) \quad c a B d a \quad B D c$

$(14,2 * 6,3 * 5,7 * 0) \quad d d b C a \quad A C b$

$(14,2 * 7,3 * 6,5 * 0) \quad d c B d a \quad A C b$

$(14,2 * 0,3 * 5,6 * 7) \quad A C D D b \quad b C a$

10 Sides

$(15) /(1-9) *$

$(13,26,4 * 9,57,8 * 0) \quad A B D b \quad$ eca Ced

$(13,2 * 6,4 * 9,57,8 * 0) \quad A B d b \quad$ eca Ced

$(14,26,37,58,9 * 0) \quad$ eeda $A C b \quad B D c$

$(14,27,38,5 * 0,69) \quad d E d a \quad A C b \quad B E c$

$(14,2 * 3,58,6 * 7,9 * 0) \quad$ eeca $A b b \quad C d d$

$(14,2 * 3,5 * 0,69,7 * 8) \quad c D c a \quad A b b \quad$ Dee

$(14,2 * 7,3 * 6,58,9 * 0) \quad$ eeda $A C b \quad B d c$

$(14,2 * 8,3 * 7,5 * 0,69) \quad d E d a \quad A C b \quad B e c$

$(15,2 * 6,38,4 * 0,7 * 9) \quad$ daBa BEC CEd

$(15,2 * 7,3 * 0,4 * 6,8 * 9) \quad B E E c \quad c D a \quad A D b$ 
$(14-2) /(1-1010) *$

$(14,26,37,5 * 9,8 * 0) \quad B D e c \quad e d a \quad A C b$

$(14,26,3 * 9,5 * 8,7 * 0) \quad A C e b \quad e d a \quad B D c$

$(14,29,3 * 6,5 * 8,7 * 0) \quad A C E b \quad e d a \quad B d c$

$(14,2 * 7,3 * 6,5 * 9,8 * 0) \quad B E d c \quad$ eda $A C b$

$(15,27,3 * 0,48,6 * 9) \quad c D e a \quad A D b \quad B E c$

$(15,28,3 * 0,4 * 6,7 * 9) \quad A D E b \quad c D a \quad B E c$

$(15,2 * 7,39,4 * 6,8 * 0) \quad e c D a \quad A D b \quad B E c$

$\left(\begin{array}{llll}1 & 8 & 17 & 12\end{array}\right) /\left(\begin{array}{llll}1 & -6 & -25 & -4\end{array}\right)$

(1 3, 25,46,70,8*9) ABCb dca Dee

$(13,2 * 5,46,70,8 * 9) \quad A B c b \quad d c a$ Dee

$(14,2 * 3,5 * 7,6 * 0,8 * 9) \quad C E E d \quad d c a \quad A b b$

$(14,2 * 3,5 * 8,69,7 * 0) \quad C d E d \quad$ eca $A b b$

$(164-10) /(1-8-1226)$

$(14,25,36,70,8 * 9) \quad d c B a \quad A C b \quad$ Dee

$(14,2 * 3,58,6 * 0,7 * 9) \quad d E c a \quad A b b \quad C E d$

$(14,2 * 3,5 * 7,69,8 * 0) \quad$ edca Abb CEd

12 Sides

$(14) /(1-8) *$

$(14,26,37,58,90,10 * 11) \quad e d a \quad A C b \quad B D c \quad E f f$

$(14,27,38,5 * 11,69,10 * 0) \quad f d a \quad A C b \quad B E c \quad D f e$

$(14,2 * 3,58,6 * 7,90,10 * 11) \quad$ eca Abb Cdd Eff

$(14,2 * 3,58,6 * 11,7 * 10,90) \quad f c a \quad A b b \quad C E d \quad D f e$

$(14,2 * 3,5 * 7,6 * 0,811,9 * 10) \quad d c a \quad A b b \quad C E d \quad E f f$

$(14,2 * 3,5 * 8,610,7 * 0,9 * 11) \quad$ eca Abb CFd DFe

$(14,2 * 8,3 * 7,5 * 11,69,10 * 0) \quad f d a \quad A C b \quad B e c \quad D f e$

$(15,27,3 * 11,48,6 * 10,9 * 0)$ fea $A D b \quad B E c \quad C f d$

$(15,2 * 8,311,4 * 7,6 * 10,9 * 0)$ fea $A D b$ BFc Ced

$(16,29,3 * 0,4 * 7,510,8 * 11) \quad c D a \quad A E b \quad B F c \quad D F e$

$(16,2 * 8,310,4 * 0,5 * 7,9 * 11) \quad d E a$ AEb BFc CFd

Growth functions for an orientable, genus 2 surface group

8 Sides

$(1 \quad 14) /(1-34) *$

$(13,24,57,60) \quad c b A B a d C D$

$(13,25,47,60) \quad c a d b A B C D$

$(13,26,47,50) \quad c a d C b A B D$

$(15,26,37,40) \quad c B a d C b A D$ 
10 Sides

(1 8) /(1 - 22)*

$(13,24,50,68,79) \quad e D E d C \quad c b A B a$

$(13,25,49,68,70) \quad c a e D E \quad A B C d b$

(13,26,48,50,79) ebABD dCEca

$(14,25,38,69,70) \quad d b A C E \quad e D c B a$

$(14,29,36,58,70) \quad b A C D E \quad e c B d a$

$(16,27,38,49,50) \quad d C b A E \quad e D c B a$

(1 1230$) /(1-20-74)$

$(13,24,57,69,80) \quad$ ecbABa $\quad d C D E$

$(13,26,49,57,80) \quad$ caedCE $A B D b$

$(13,27,49,50,68) \quad c a d C e D \quad A B E b$

$(18-2) /(1-2414) *$

$(13,25,48,69,70) \quad d b A B C E \quad e D c a$

$(13,27,48,59,60) \quad d C b A B E \quad e D c a$

$(14,26,39,58,70) \quad e b A C d a \quad c B D E$

$\left(\begin{array}{lll}1 & 10 & 2\end{array}\right) /(1-286) *$

$(13,24,58,69,70) \quad e D c b A B a \quad d C E$

$(13,25,47,69,80) \quad d b A B C D E \quad$ eca

$(13,26,47,59,80) \quad d C b A B D E \quad$ eca

$(13,26,48,59,70) \quad$ ebABDca $d C E$

$(14,26,38,59,70) \quad$ daebACE $B D c$

$(14,27,38,59,60) \quad$ daeDcBE $A C b$

12 Sides

$(16) /(1-18) *$

$(13,25,46,79,811,100) \quad e D E F \quad f d c a \quad A B C b$

$(13,26,410,57,811,90) \quad e d C F \quad f E c a \quad A B D b$

$(13,28,46,511,79,100) \quad d C D F$ feca $A B E b$

$(13,28,410,511,60,79) \quad d C f E \quad e D c a \quad A B F b$

$(14,26,311,59,710,80) \quad c B D F \quad f E d a \quad A C e b$

$(14,29,36,510,711,80) \quad e c B F \quad f E d a \quad A C D b$

$(17) /(1-23) *$

$(14,25,36,710,811,90) \quad f E d c B a \quad e D F \quad A C b$

$(14,27,38,510,611,90) \quad f c B E d a \quad e D F \quad A C b$

$(14,27,310,58,611,90) \quad e D b A C F \quad f d a \quad B E c$

$(14,211,36,58,710,90) \quad b A C D E F \quad f d a \quad B e c$

(1 11124$) /(1-19-60)$

$(13,24,58,610,711,90) \quad f c b A B a \quad e D F \quad C E d$

(1 3,2 8,47,5 10,6 11,90) fbABca eDF CEd

$(14,28,39,511,60,710) \quad$ daeDfE $A C b \quad B F c$ 
$(16-2) /(1-2014) *$

$(13,25,410,69,711,80)$

$(13,26,48,511,710,90)$

$(13,26,49,511,710,80)$

$(13,27,49,510,60,811)$

$A B C d b \quad f E c a \quad e D F$

$A B D e b \quad d C E F \quad f c a$

$A B D e b \quad f E c a \quad d C F$

fbABE eDca CFd

$\left(\begin{array}{lll}1 & 8 & 6\end{array}\right) /(1-22-6) *$

$(13,25,48,610,711,90)$

$A B C E d b \quad e D F \quad f c a$

$(13,26,48,510,711,90) \quad e b A B D F \quad f c a \quad C E d$

$(13,27,48,510,611,90) \quad A B E d C b \quad e D F \quad f c a$

$(14,27,310,59,611,80) \quad f b A C d a \quad e D F \quad B E c$

$(15,27,39,411,610,80) \quad$ fbADea $\quad d C F \quad B E c$

$\left(\begin{array}{llll}1 & 12 & 37 & 20\end{array}\right) /(1-14-101-12)$

$(13,24,50,68,710,911) \quad c b A B a$ DEFe fdC

$\left(\begin{array}{llll}1 & 10 & 26 & 22\end{array}\right) /(1-16-64-34)$

$(13,25,49,68,711,100) \quad A B C d b \quad e D E F \quad f c a$

$(13,27,49,511,68,100) \quad d C e D F \quad A B E b \quad f c a$

$\left(\begin{array}{llll}1 & 10 & 16 & -30\end{array}\right) /(1-16-68118)$

$(13,24,50,69,710,811) \quad c b A B a \quad f E d C \quad D F e$

$(189-12) /(1-18-3352)$

$(13,25,46,710,811,90) \quad f E d c a \quad A B C b \quad e D F$

$(13,27,410,511,68,90) \quad$ feDca $A B E b \quad d C F$ $\left(\begin{array}{llll}1 & 6 & 0 & 10\end{array}\right) /\left(\begin{array}{llll}1 & -20 & 12 & -34\end{array}\right) *$

$(14,25,38,610,711,90) \quad A C E d b \quad f c B a \quad e D F$

$(14,26,39,510,711,80) \quad e b A C F \quad f E d a \quad B D c$

$(14,26,310,58,711,90) \quad e b A C F \quad B D E c \quad f d a$

$(14,26,311,58,710,90) \quad c B D E F \quad A C e b \quad f d a$

$(14,28,39,510,611,70) \quad e D c B F \quad f E d a \quad A C b$

$(14,210,37,59,611,80) \quad f c B d a \quad A C E b \quad e D F$

\section{Sides}

(1 6)/(1 - 18)*

$(13,26,49,512,711,813,100) \quad A B D e b \quad f E G \quad g c a \quad C F d$ $(13,27,49,511,613,812,100) \quad A B E f b \quad e D G \quad$ gca $C F d$

$\left(18 \frac{16}{1}\right) /(1-14-42)$

$(14,25,310,69,712,813,110) \quad g c B a \quad A C d b \quad f E G \quad D F e$ $(14,29,310,512,613,70,811) \quad e D g F \quad f E d a \quad A C b \quad B G c$ 
$(166) /(1-16-10) *$

$(13,25,46,70,810,912,1113)$

$A B C b$ EFGf geD dca

$(13,26,411,57,810,913,120)$

fEFG ABDb gca Ced

$(13,29,46,512,70,810,1113)$

$A B F b \quad C D G d$ gfE eca

$(14,26,312,59,711,813,100)$

$A C e b \quad B D F c$ fEG $g d a$

$(14,27,38,510,613,912,110)$

eDFG BEfc gda ACb

$(14,27,312,510,613,811,90)$

$g F d a \quad A C f b \quad e D G \quad B E c$

$(14,211,37,59,612,813,100)$

$f c B G \quad A C E b \quad g d a \quad D F e$

$(15,26,39,412,711,813,100)$

$(15,27,310,413,611,812,90)$

gcBa ADeb fEG CFd

$(15,29,313,410,611,712,80)$

gFea $A D f b \quad d C G \quad B E c$

$(14-4) /(1-1822) *$

$(14,25,36,70,811,912,1013)$

gFea CfEd $c B G \quad A D b$

$(14,26,37,512,811,913,100)$

gFeD $\quad d c B a \quad A C b \quad E G f$

$(14,26,311,512,710,813,90)$

$g F d a$ BDec fEG $A C b$

$(14,27,312,59,613,811,100)$

$g F d a \quad A C e b \quad f E G \quad B D c$

$(14,28,39,511,612,70,1013)$

eDFG ACfb gda BEc

gcBF fEda ACb DGe

$(14,212,37,59,613,811,100) \quad e D F G \quad A C E b \quad g d a \quad B f c$

$\left(\begin{array}{llll}1 & 9 & 23 & 22\end{array}\right) /(1-13-57-38)$

$(13,25,46,710,812,913,110) \quad g d c a \quad A B C b \quad f E G \quad D F e$

$(13,29,47,512,613,810,110) \quad g f c a \quad A B F b \quad e D G \quad C E d$

$\left(\begin{array}{llll}1 & 11 & 31 & 26\end{array}\right) /(1-13-81-38)$

$(13,24,50,69,711,812,1013) \quad c b A B a \quad g d C \quad D F e \quad E G f$

$(177-14) /(1-15-3362)$

$(13,27,410,513,68,912,110) \quad d C F G \quad A B E b \quad$ gca $D f e$

$(177-6) /(1-17-2534)$

$(14,25,36,710,812,913,110) \quad g d c B a \quad f E G \quad A C b \quad D F e$

$(14,28,39,512,613,710,110) \quad g f E d a \quad e D G \quad A C b \quad B F c$

$(15-16) /(1-1915-26) *$

$(14,26,37,510,812,913,110) \quad B D F e c$ fEG $g d a \quad A C b$

$(14,26,39,510,712,813,110) \quad A C F e b \quad f E G \quad g d a \quad B D c$

$(14,27,311,59,612,813,100) \quad f b A C G$ gda BEc DFe

$(14,28,39,510,612,713,110) \quad B F e D c$ fEG $g d a \quad A C b$

$(14,212,36,59,711,813,100) \quad A C D F b$ fEG $g d a \quad B e c$

$(15,28,312,49,611,713,100) \quad g d C e a \quad f E G \quad A D b \quad B F c$

$\left(\begin{array}{lllll}1 & 8 & 17 & 16 & 28\end{array}\right) /(1-14-43-18-76)$

$(13,26,410,57,812,913,110) \quad$ CFed ABDb fEG gca

(1 3, 2 7,4 10,5 12,68,913,110) feDG ABEb gca $C F d$

$(13,28,410,512,613,79,110) \quad C f E d \quad A B F b \quad e D G \quad g c a$ 
$(1811-1616) /(1-14-5186-92)$

$(13,25,46,70,811,912,1013) \quad g F e D \quad A B C b \quad d c a \quad E G f$

$\left(\begin{array}{lllll}1 & 8 & 12 & 0 & 6\end{array}\right) /(1-16-3616-26)$

$(13,25,410,69,712,813,110) \quad A B C d b \quad f E G$ gca DFe $(13,29,47,511,612,80,1013) \quad g b A B F$ fca CEd DGe

\section{Sides}

$\left(\begin{array}{lll}1 & 5 & 4\end{array}\right) /(1-15-4) *$

$(14,26,37,58,912,1014,1115,130)$

$(14,210,311,58,614,715,912,130)$

heda $\quad g F H \quad A C b \quad B D c \quad E G f$

hgda $f E H \quad A C b \quad B G c \quad D F e$

$(15,27,310,414,611,813,915,120)$

$A D f b \quad g F H$ hea $B E c \quad C G d$

$(15,27,314,48,611,913,1015,120)$

$B E G c \quad g F H$ hea $A D b \quad C f d$

$(15,28,315,49,611,713,1014,120)$

CBFH hea $A D b$ Cgd EGf

$(15,29,314,410,611,713,815,120)$

$C f E d \quad g F H$ hea $A D b \quad B G c$

$\left(\begin{array}{llll}1 & 9 & 26 & 32\end{array}\right) /(1-11-60-68)$

$(13,26,412,57,811,914,1015,130)$

$A B D b \quad g F H$ hca Ced EGf

$(13,210,47,513,614,80,911,1215)$

$A B G b$ hgF fca CEd DHe

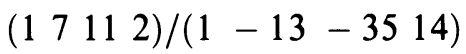

$(13,27,411,514,68,913,1015,120)$

$A B E b \quad g F H$ hca $C G d$ Dfe

$(13,28,411,513,615,79,1014,120)$

$A B F b$ eDH hca CGd Egf

$(165-8) /(1-14-2340)$

$(14,28,39,512,615,710,1114,130) \quad e D G H \quad h d a$ ACb BFc Egf

$(14-12) /(1-1611-12) *$

$(14,27,38,511,614,913,1015,120)$

$B E f c \quad g F H \quad h d a \quad A C b \quad D G e$

$(14,27,313,510,614,812,915,110)$

$A C f b \quad g F H$ hda $B E c$ DGe

$(14,28,39,511,613,715,1014,120)$

$B F g c \quad f E H$ hda $A C b$ DGe

$(14,213,37,510,614,812,915,110)$

$A C E b \quad g F H$ hda $B f C$ DGe

$\left(\begin{array}{lllll}1 & 9 & 24 & 25 & 34\end{array}\right) /(1-11-62-37-86)$

$(13,25,46,70,811,913,1014,1215) \quad A B C b$ heD dca EGf FHg

$(179-86) /(1-13-3950-46)$

$(14,25,36,70,811,913,1014,1215) \quad d c B a$ heD ACb EGf $F H g$

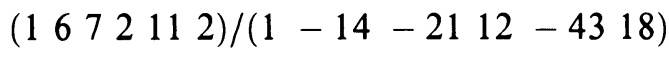

$(14,26,37,512,811,914,1015,130)$

BDec $\quad g F H \quad h d a \quad A C b \quad E G f$

$(14,26,311,512,710,814,915,130) \quad A C e b \quad g F H$ hda $B D c \quad E G f$

$(14,29,310,512,614,715,811,130)$

$D g F e \quad f E H$ hda $A C b \quad B G c$ 


\section{Sides}

$$
(14) /(1-14) *
$$

$(14,26,37,58,90,1013,1115,1216,1417)$ ifE eda ACb BDc FHg GIh

$(14,27,38,514,69,1013,1116,1217,150) \quad h g I$ ida $A C b \quad B E c \quad D f e \quad F H g$

$(14,28,39,513,616,710,1115,1217,140) \mathrm{hgI}$ ida $A C b \quad B F c$ DHe Egf

$(14,29,310,513,615,717,811,1216,140) \quad f E I$ ida $A C b \quad B G c$ DHe Fhg

$(14,211,312,58,615,716,90,1013,1417)$ ihG gda ACb BHc DFe EIf

$(15,28,315,49,612,716,1014,1117,130) \quad h g I$ iea $A D b \quad B F c \quad C g d \quad E H f$

$(15,29,316,410,612,714,817,1115,130) \quad g F I$ iea $A D b \quad B G c$ Chd EHf

$(16,29,314,417,510,712,815,1116,130) \quad d C I$ ifa $A E b \quad B G c$ Dhe FHg

\section{REFERENCES}

1. M. Benson, Growth series of finite extensions of $\mathbb{Z}^{n}$ are rational, Invent. Math. 73 (1983), 251-269.

2. J. W. Cannon, The combinatorial structure of cocompact discrete hyperbolic groups, Geom. Dedicata 16 (1984), 123-148.

3. - The growth of the closed surface groups and the compact hyperbolic Coxeter groups, unpublished manuscript.

4. W. J. Floyd and S. P. Plotnick, Growth functions on Fuchsian groups and the Euler characteristic, Invent. Math. 88 (1987), 1-29.

5. __ Symmetries of planar growth functions, Invent. Math. 93 (1988), 501-543.

6. F. R. Gantmacher, The theory of matrices, vol. 1, Chelsea, New York, 1977.

7. P. Wagreich, The growth function of a discrete group, Proc. Conf. Algebraic Varieties with Group Actions, Lecture Notes in Math., Vol. 956, Springer, Berlin, Heidelberg, New York, 1982, pp. 125-144.

Department of Mathematics, Virginia Polytechnic Institute and State University, BLACKSBURG, VIRGINIA 24061-0123 